\title{
Mechanisms of Multivesicular Body Biogenesis and Exosome Release
}

\author{
Dissertation \\ for the award of the degree \\ "Doctor of Philosophy" (Ph.D.) \\ Division of Mathematics and Natural Sciences \\ of the Georg-August-Universität Göttingen
}

submitted by

Chieh Hsu

born in

Taipei, Taiwan

Göttingen 2009 
Members of the Thesis Committee:

Prof. Dr. Mikael Simons, Reviewer

Max Planck Institute of Experimental Medicine

Department of Neurology, University of Göttingen

Prof. Dr. Reinhard Jahn, Reviewer

Department of Neurobiology, Max Planck Institute for Biophysical Chemistry Prof. Klaus-Armin Nave, Ph.D.

Department of Neurogenetics, Max Planck Institute of Experimental Medicine

Date of the oral examination: 8 February, 2010 


\section{Affidavit}

I hereby declare that this PhD thesis "Mechanisms of Multivesicular Body Biogenesis and Exosome Release" has been written independently with no other aids or sources than quoted.

Chieh Hsu

December, 2009

Göttingen, Germany 


\section{Related publications}

Katarina Trajkovic*, Chieh Hsu*, Salvatore Chiantia, Lawrence Rajendran, Dirk Wenzel, Felix Wieland, Petra Schwille, Britta Brügger and Mikael Simons. (2008) Ceramide Triggers Budding of Exosome Vesicles into Multivesicular Endosomes. Science; 319:12441247

*These authors contributed equally to this work.

Chieh Hsu, Yuichi Morohashi, Shin-ichiro Yoshimura, Natalia Manrique-Hoyos, Marcel A. Lauterbach, Mostafa Bakhti, Mads Grønborg, Wiebke Möbius, Francis A. Barr and Mikael Simons. (2010) Regulation of exosome secretion by Rab35 and its GTPaseactivating proteins TBC1D10A-C. J. Cell Biol.; 189:223-232 


\section{Contents}

Contents vii

List of Figures $\ldots \ldots \ldots \ldots \ldots \ldots \ldots \ldots \ldots$ xi

Abbreviations $\quad$ xiii

$\begin{array}{ll}\text { Acknowledgements } & \text { xv }\end{array}$

Abstract $\quad$ xvii

Zusammenfassung xix

1 Introduction $\quad 1$

1.1 Exosomes . . . . . . . . . . . . . . . . . . . . 1

1.1.1 Structure and composition of exosomes . . . . . . . . . . . . 2

1.1.2 Functions of exosomes . . . . . . . . . . . . . . . . . . 4

1.1.3 Application of exosomes . . . . . . . . . . . . . . . 6

1.2 Multivesicular Bodies . . . . . . . . . . . . . . . . . 7

1.2.1 Endosomal trafficking . . . . . . . . . . . . . . . 7

1.2.2 Late endosomes, lysosomes and lysosome related organelles . . . . . 10

1.2.3 Biogenesis of intraluminal vesicles I - the ESCRT machinery . . . . . 12

1.2.4 Biogenesis of intraluminal vesicles II - lipid dependent machanisms . 15

1.3 Biogenesis of exosomes . . . . . . . . . . . . . . . . . . . 16

1.3.1 Retrovirus budding . . . . . . . . . . . . . . . . 16

1.3.2 Biogenesis of exosomes . . . . . . . . . . . . . . . . 17

1.4 The Rab GTPases . . . . . . . . . . . . . . . . . . . . . . . . . . 18

1.4.1 Rabs in intracellular trafficking . . . . . . . . . . . 18

1.4.2 Rabs in tethering factor recruitment . . . . . . . . . . . . 21

1.5 The Proteolipid Protein . . . . . . . . . . . . . . . . . . . . 23 
1.6 Goal of the project . . . . . . . . . . . . . . . . . 24

2 Materials and Methods $\quad 25$

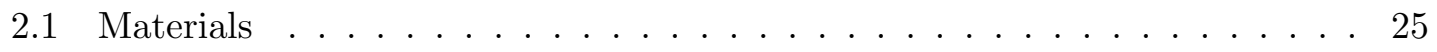

2.1.1 Chemicals and comsumables . . . . . . . . . . . 25

2.1 .2 Cell lines . . . . . . . . . . . . . . . . . . . . 25

2.1.3 Antibodies and other proteins . . . . . . . . . . . . . 26

2.1 .4 Plasmids . . . . . . . . . . . . . . . . 26

2.1.4.1 Previously published plasmids . . . . . . . . . 26

2.1.4.2 Self contructed plasmids . . . . . . . . . . . . 27

2.1.5 Oligonucleotides and siRNA reagents . . . . . . . . . . . 27

2.1.5.1 DNA primers . . . . . . . . . . . . 27

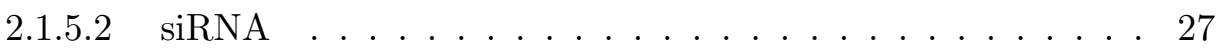

2.1 .6 Commercial Kits . . . . . . . . . . . . . . . . . . . . 27

2.1 .7 Buffers, soultions, sera and media . . . . . . . . . . . . . 28

2.1.7.1 Commercial media . . . . . . . . . . . . . . . 28

2.1.7.2 PBS (phophate buffered saline) . . . . . . . . . . 28

2.1.7.3 SATO medium . . . . . . . . . . . . . . . . 29

2.1.7.4 Mowiol solution for immunocytochemistry . . . . . . . 30

2.1 .8 Softwares . . . . . . . . . . . . . . . . . 30

2.2 Methods . . . . . . . . . . . . . . . . . . . . 31

2.2.1 Cell culture, transfection, and RNA interference . . . . . . . . 31

2.2.1.1 Primary oligodendrocyte culture . . . . . . . . . . . 31

2.2.1.2 Growth and maintenance of cell lines . . . . . . . . . . . 31

2.2.1.3 Freezing and thawing of cells . . . . . . . . . . . 31

2.2.1.4 Transfection . . . . . . . . . . . . . . 32

2.2.1.5 RNA interference . . . . . . . . . . . . . 32

2.2 .2 Molecular biology . . . . . . . . . . . . . . . . . . 33

2.2.2.1 Plasmid isolation from E. Coli . . . . . . . . . . . . 33

2.2.2.2 Restriction enzyme digestion . . . . . . . . . . 33

2.2 .2 .3 DNA ligation . . . . . . . . . . . . . . . 34

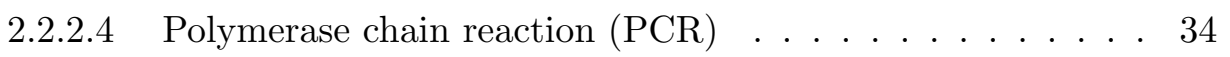

2.2.2.5 Determine of DNA concentration . . . . . . . . . . . 35

2.2.2.6 Transformation of E. Coli cells . . . . . . . . . . . . 35 
2.2.2.7 DNA sequencing and oligonucleotide synthesis . . . . . 36

2.2 .3 Protein biochemistry . . . . . . . . . . . . . . . . 36

2.2 .3 .1 SDS-PAGE . . . . . . . . . . . . . 36

2.2.3.2 Western Blot . . . . . . . . . . . . . . 37

2.2 .4 Exosome purification . . . . . . . . . . . . . . 38

2.2 .5 Immunocytochemistry and microscopy . . . . . . . . . . . . 39

2.2.5.1 Immunocytochemistry . . . . . . . . . . . . . . . 39

2.2.5.2 Confocal Microscopy . . . . . . . . . . . . . . . . . . 39

2.2.5.3 TIRF Microscopy . . . . . . . . . . . . . . . 40

2.2.5.4 Electron microscopy . . . . . . . . . . . . . . . . 40

2.2 .6 Image Analysis . . . . . . . . . . . . . . . . . . . . . . . . 41

2.2.6.1 Colocalization analysis on endosomal membrane . . . . . 41

2.2.6.2 Quantification of signals within the endosomal lumen and on the limiting membrane . . . . . . . . . . . . . . . 41

2.2.6.3 Vesicle tracking . . . . . . . . . . . . . . . . 42

2.2.6.4 Correlation analysis . . . . . . . . . . . . . . 42

2.2 .7 EGF degradation . . . . . . . . . . . . . . . . . 43

2.2 .8 Myelin preparation . . . . . . . . . . . . . . . . 43

3 Results $\quad 45$

3.1 Ceramide triggers budding of vesicles into MVBs for the release as exosomes 45

3.1.1 Oli-neu cells secrete exosomes . . . . . . . . . . . . . . . . 45

3.1.2 Formation of endosomal subdomains . . . . . . . . . . . . . . 46

3.1.3 ESCRT-independent formation of exosomes . . . . . . . . . . . . 49

3.1.4 A role of ceramide in the formation of exosomes . . . . . . . . 56

3.2 Regulation of exosome secretion by Rab35 and its GAPs . . . . . . . . . . 60

3.2.1 Proteome analysis of Rab GTPases in exosomes . . . . . . . . . . . . 60

3.2 .2 A Rab GAP screen in exosome secretion . . . . . . . . . . . . . 60

3.2.3 Identification of Rab35 as the target Rab of TBC1D10A-C . . . . . 62

3.2.4 A role for Rab35 in exosome secretion . . . . . . . . . . . . . 63

3.2.5 Localization of Rab35 . . . . . . . . . . . . . . . . . . . 64

3.2 .6 Function of Rab35 . . . . . . . . . . . . . . . . 65

3.2.7 GTPase-defective Arf6 ${ }^{\mathrm{Q} 67 \mathrm{~L}}$ reduces exosome secretion $\ldots . . . .70$ 
4.1 Mechansims of exosome biogenesis . . . . . . . . . . . . . . . 73

4.1.1 ESCRT-independent release of exsomes . . . . . . . . . . . 73

4.1.2 Ceramide in exosome biogenesis . . . . . . . . . . . . . . . 75

4.1.3 Sorting of cargo into MVBs . . . . . . . . . . . . . . 77

4.1.4 Existence of different exosome populations . . . . . . . . . . 79

4.2 Rab35 and endosomal trafficking . . . . . . . . . . . . . . . 80

4.3 MVB to plasma membrane trafficking . . . . . . . . . . . . . . . 82

4.4 Function of exosomes . . . . . . . . . . . . . . . . . . . 84

5 Summary and conclusion $\quad 87$

$\begin{array}{lr}\text { A Oli-neu exosomal proteins } & 89\end{array}$

$\begin{array}{lr}\text { B Source codes } & 97\end{array}$

B.1 Calculation of correlation coefficient between two channels . . . . . . . . . 97

B.2 Calculation of correlation coefficient within image series . . . . . . . . . 102

$\begin{array}{ll}\text { Bibliography } & 107\end{array}$

$\begin{array}{lr}\text { Curriculum Vitae } & 131\end{array}$ 


\section{List of Figures}

1.1 Pathways of endosomal trafficking . . . . . . . . . . . . . . . 8

1.2 Composition and molecular interactions of the ESCRT machinery . . . . . 14

1.3 Localization and function of Rab GTPases . . . . . . . . . . . . . . . . . 19

1.4 A model for tethering during membrane traffic . . . . . . . . . . . . 22

3.1 PLP was detected in exosomes biochemically . . . . . . . . . . . . 47

3.2 Ultrastructure of the exosomes secreted by Olin-neu cells . . . . . . . . . . 47

3.3 Endogenous and exogenous proteins in exosomes . . . . . . . . . . . 48

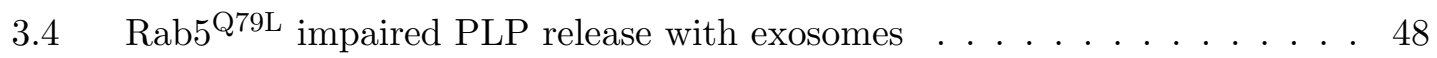

3.5 Colocalization of EGF and HA-Hrs on endosomes . . . . . . . . . . . . . 49

3.6 Endosomal subdomain structure . . . . . . . . . . . . . . . . 50

$3.7 \quad$ ESCRT-independent intrluminal transport of PLP . . . . . . . . . . . 52

3.8 EGF degradation after treatment with siRNA against Hrs, Tsg101 and Alix 53

3.9 Tsg101-dependent transport of VSV-G . . . . . . . . . . . . 53

3.10 Colocalization of PLP and Lamp-1 after treatment with siRNA against Hrs, Tsg101 and Alix . . . . . . . . . . . . . . . . . 54

3.11 ESCRT-independent exosome release of PLP . . . . . . . . . . . . 55

3.12 Tsg101 overexpression does not affect exosome release of PLP . . . . . . . 55

3.13 Lipid analysis of exosomes . . . . . . . . . . . . . . . . . . 56

3.14 Exosomal release of PLP was reduced with inhibition of nSMase . . . . 57

3.15 NSMase dependent intraluminal transport of PLP . . . . . . . . . . . 58

3.16 Ceramide formation triggers intraluminal budding of vesicles on GUVs . . 59

3.17 Western blot analysis of Rabs in exosomes . . . . . . . . . . . . . 61

3.18 Screen of a Rab GAP library identifies the TBC1D10 family as regulators of exosome secretion . . . . . . . . . . . . . . . . . 62

3.19 Identification of Rab35 as the target of TBC1D10A-C . . . . . . . . . . 63

3.20 Inhibition of Rab35 function reduces exosome secretion of PLP . . . . . . 64 
3.21 Subcellular localization of Rab35 . . . . . . . . . . . . . . . 66

3.22 TBC1D10B localizes to the plasma membrane . . . . . . . . . . . 67

3.23 Rab35 and MVBs in myelin . . . . . . . . . . . . . . . 67

3.24 Rab7 $^{\mathrm{T} 22 \mathrm{~N}}$ changed the colocalization of PLP and Lamp-1 . . . . . . . . 68

3.25 Inhibition of Rab7, but not Rab35 inhibits EGF degradation . . . . . . 68

3.26 Knock-down of Rab35 expression accumulated PLP containing endosomes 69

3.27 Rab35 ${ }^{\mathrm{Q} 67 \mathrm{~A}}$ reduced the mobility of endosomes . . . . . . . . . . . 70

3.28 Rab35 regulated endosome attachment to the plasma membrane . . . . . 71

3.29 Effect of Arf6 ${ }^{\mathrm{Q} 67 \mathrm{~L}}$ on PLP localization and exosome release $\ldots . . . . .72$ 


\section{Abbreviations}

$\begin{array}{ll}\text { APC } & \text { Antigen presenting cell } \\ \text { APP } & \text { Amyloid precursor protein } \\ \text { APS } & \text { Ammonium persulfate } \\ \text { CNS } & \text { Central nervous system } \\ \text { CSF } & \text { Cerebrospinal fluid } \\ \text { EAE } & \text { Experimental autoimmune encephalomyelitis } \\ \text { EDTA } & \text { Ethylenediaminetetraacetic acid } \\ \text { EGF } & \text { Epidermal grotwh factor } \\ \text { EGFR } & \text { Epidermal grotwh factor receptor } \\ \text { ER } & \text { Endoplasmic reticulum } \\ \text { ESCRT } & \text { Endosomal sorting complex required for transprort } \\ \text { GAP } & \text { GTPase activating protein } \\ \text { GDI } & \text { GDP dissociation inhibitor } \\ \text { GDP } & \text { Guanosine diphosphate } \\ \text { GEF } & \text { Guanin nucleotide exchange factor } \\ \text { GPI } & \text { Glycosyl phosphatidylinositol } \\ \text { GTP } & \text { Guanosine triphosphate } \\ \text { GTPase } & \text { Guanine triphosphatases } \\ \text { GUV } & \text { Giant Unilamellar vesicle } \\ \text { HEPES } & \text { 4-(2-hydroxyethyl)-1-piperazineethanesulfonic acid } \\ \text { HIV } & \text { Human immunodeficiency virus } \\ \text { hr } & \text { Hour(s) } \\ \text { HRP } & \text { Lorse radish peroxidase } \\ \text { LBPA } & \text { Major histocompatibility complex } \\ \text { M6PR } & \text { MHC }\end{array}$


$\min$

MVB

nSMNase

PAGE

PBS

PCR

PLL

PLP

PS

Rab

RT

SDS

sec

SMNase

SNARE

TEMED

TGN

TIRF

TNF

VLP

VSV
Minute(s)

Multivesicular body

neutral SMNase

Polyacriamide gel electrophoresis

Phosphate buffered saline

Polymerase chain reaction

Poly-L-lysine

Proteolipid protein

Phosphatidylserine

Ras like protein in brain

Room Temperature

Sodium dodecyl sulphate

Second(s)

Sphingomyelinases

Soluble NSF attachment protein receptor

N'N'N'-tetramethylethylene diamine

Trans-Golgi networks

Total internal reflection fluorescence

Tumor necrosis factor

Virus like particle

Vesicular stomatitis virus 


\section{Acknowledgements}

This work would not have been possible without Prof. Mikael Simons, my direct supervisor, who initiated and continuously supported this project. I enjoyed exploring the fields related to this study with his scientific guidance and technical advisory.

I appreciate Prof. Klaus-Armin Nave and Prof. Reinhard Jahn for being the members for my PhD committee and the fruitful discussions during the meetings.

In recent days, scientific research requires team works with people having different specialties. I sincerely thank the collaborators: Katarina Trajkovic who contributed largely on MVB biogenesis; Francis A. Barr, Yuichi Morohashi and Shin-ichiro Yoshimura for constructing the Rab-GAP library and performing GTPase activity screen; Petra Schwille and Salvatore Chiantia for in vitro GUV experiments; Britta Brügger for lipidomics; Mads Grønberg for proteomics; Wiebke Möbius and Dirk Wenzel for electron microscopy; Marcel A. Lauterbach for endosome tracking algorithm; Natalia Manrique-Hoyos for myelin sub-fractioning; Mostafa Bakhti for participating the study of Arf6 and Rab35 interactions.

I thank Giselheid Schulz, Anja Schneider and Tobias Kohl, whom I learned details of manipulating cell culture, biochemical analysis and TIRF microscope from. I thank Katrin Strauß and Sebastian Schmitt for proofreading my German abstract.

I thank the graduate programs GRK521 and GGNB, especially Prof. Doenecke, the GRK521 coordinator, for financial and administrative support during my doctoral curriculum. I am grateful for the softwares developed by the open source community that established the environment I worked on - from the operating system to scientific analytical tools. 
I would like to mention the colleges and friends in AG Simons - Giselheid Schulz, Anja Schneider, Katarina Trajkovic, Dirk Fitzner, Angelika Kippert, Larisa Yurlova, Holger Budde, Katrin Strauß, Mareike Schnaars, Mostafa Bakhti, Natalia Manrique-Hoyos, Sebastian Schmitt, Shweta Aggarwal and Johannes Zimmermann — for the great atmosphere and idea exchange.

I thank Gabriel Mora, Alexandra Andreou, Ioanna Bethani, Ye Chen (陈业), Tsai-Wen Chen (陳摘文) and many, many friends who accompanied me during the Göttingen years for making the small town my second home.

I am grateful for everything that my mother, Piao-Yu Huang (黃漂鈺), and my father, Chung-Chih Hsu (徐忠治), have done for me. I thank them for their unlimited love, support and trust.

My special thanks go to Ju-yi Chou (Yuta, Roshnii, 周如怡), Larisa Yurlova, and ChihHan Chang (張智涵) for strengthening me during the tough periods as well as sharing the delight and enjoyment of my life. 


\section{Abstract}

Exosomes are small vesicles with a diameter of approximately 50-100 $\mathrm{nm}$ that are secreted by a number of different cells and function in a multitude of intercellular signaling processes. Exosomes are thought to derive from release of intraluminal vesicles (ILVs) in multivesicular bodies (MVBs) after the fusion of MVBs with the plasma membrane.

To understand the little explored molecular details of how cargoes are sorted to these subsets of ILVs in MVBs and secreted with exosomes, the transport of proteolipid protein (PLP) in Oli-neu cells, an oligodendroglial precursor cell line, was studied. We found that PLP was transported to distinct subdomains of the endosomal membrane and that the transfer of PLP into the lumen of endosomes did not depend on the function of the endosomal sorting complex required for transport (ESCRT) machinery but required ceramide formation. The purified exosomes were enriched with ceramide and inhibition of neutral sphingomyelinase activity reduced the release of exosomes. Sphingomyelin hydrolysis on the outer leaflet of phase separated giant unilamellar vesicles resulted in intraluminal budding from the lipid ordered phase. These results identified a novel lipid-dependent pathway for cargo transport to the ILVs in MVBs for exosomes formation.

Rab family guanosine triphosphatases (GTPases) are regulators involved in intracellular membrane trafficking. A screen for the effects of Rab GTPase activating proteins (GAPs) on exosome release was performed. Expression of TBC1D10B, RN-tre, TBC1D10A, TBC1D10C and TBC1D15 reduced exosome secretion in a catalytic activity dependent manner. Rab35 was identified to be the target of TBC1D10A-C. Inhibition of Rab35 function impaired exosome secretion, induced accumulation of PLP containing endosomes and increased the mobility of endosomes closed to the plasma membrane, suggesting a function in MVB tethering. Furthermore, Rab35 was biochemically purified together with non-compact myelin, where MVBs were observed, providing a basis for understanding the biogenesis and functions of exosomes in the central nervous system. 


\section{Zusammenfassung}

Exosomen sind kleine Vesikel mit einem Durchmesser von ca. 50-100 nm, die von vielen Zellarten sezerniert werden und verschiedene interzelluläre Signalprozesse koordinieren. Nach der Fusion von multivesikulären Endosomen (MVE) mit der Plasmamembran werden die intraluminalen Vesikel (ILV) als Exosomen in den extrazellulären Raum freigesetzt.

Welchen molekularen Mechanismen der Sortierung bestimmter Moleküle in die ILV der MVE zugrunde liegen, ist jedoch nicht vollständig geklärt. Um diese Mechanismen besser zu verstehen, wurde der Transport von Proteolipidprotein (PLP) in Oli-neu Zellen, einer Zelllinie von Oligodendrozytenvorläuferzellen untersucht. Es konnte gezeigt werden, dass PLP in eigene Subdomänen der Endosomenmembran tranportiert wird. Der Transport von PLP ins Lumen der Endosomen ist dabei nicht abhängig von der ESCRT-(endosomal sorting complex required for transport)-Maschinerie ab, sondern bedarf der Bildung von Ceramiden. Es konnte eine Anreicherung von Ceramiden in Exosomen nachgewiesen werden; gleichzeitig reduzierte die Hemmung von neutralen Sphingomyelinasen die Freisetzung von Exosomen. Daher wurden GUVs (giant unilamelar vesicles) mit Sphingomyelin hergestellt, in denen zwei unterschiedlichen Phasen vorlagen. Die Hydrolyse von Sphingomyelin an der äußeren Schicht der GUV bewirkte eine Abschnürung intraluminaler Vesikel aus der gelartigen Lipidphase. Es wurde damit ein bisher unbekannter, lipidabhängiger Weg des Proteintranports in die ILV der MVE für Bildung von Exosomen gefunden.

Rab Guanosin triphosphatasen (GTPases) sind Regulatoren des intrazellulären Membransortierung. Um die Wirkung der GAPs (GTPase activating proteins) auf Exosomenfreizetzung zu untersuchen, wurde ein Screen eingesetzt. Die Expression von TBC1D10B, RNtre, TBC1D10A, TBC1D10C und TBC1D15 reduzierte die Freisetzung von Exosomen in Abhängigkeit von deren katalytischer Aktivität. In einem weiteren biochemischen 
Screen wurde Rab35 als Kandidatenprotein identifiziert, dessen die GTP-hydrolyse von TBC1D10A-C spezifisch stimuliert wurde. Eine Hemmung der Funktion von Rab35 blockierte die Exsomenfreizetzung, induzierte die Akkumulation von PLP in den Endosomen und erhöhte die Endosomenmobilität an der Plasmamembran. Diese Ergebnisse zeigen eine bisher unbekannte Funktion von Rab35 bei der Regulation von der Docking/Tethering zwischen die MVB und der Plasmamembran. Dass Rab35 zusammen mit nichtkompaktem Myelin, welches MVE beinhaltet, aufgereinigt werden kann, lässt vermuten, dass die Biogenese von Exosomen auch in der Funktion des zentralen Nervensystems eine Rolle spielt. 


\section{Chapter 1}

\section{Introduction}

\subsection{Exosomes}

Exosomes were described first in reticulocytes more than 25 years ago. Exosomes are small membrane vesicles of a diameter of 50-100 $\mathrm{nm}$. They are thought to form within the endosomal system when the limiting membrane of endosomes buds into the lumen to generate small interluminal vesicles (ILVs) within the multivesicular endosomes/bodies (MVBs). These ILVs are secreted into the extracellular space when MVBs fuse with the plasma membrane to release its content into the extracellular medium (Pan \& Johnstone, 1983; Harding et al., 1983). The secreted vesicles can be purified biochemically by sequential centrifugation steps of the extracellular medium. The exosomal membrane fraction is recovered in a final $100000 \mathrm{~g}$ centrifugation step (Pan \& Johnstone, 1983). The $100000 \mathrm{~g}$ pellet from reticulocyte culture contains specific proteins, such as transferrin receptor and HSP 70, that are released in association with exosomes during reticulocyte differentiation (Pan \& Johnstone, 1984; Davis et al., 1986). In these initial landmark papers the authors speculated that exosomes are vehicles to remove obsolete proteins that occur during the reorganization of the membrane during cellular differentiation. Shortly later, a retrograde pathway from the MVBs to the plasma membrane was proposed as a major pathway pathway for the secretion of vesicles that were termed "exosomes" ${ }^{1}$ (Johnstone et al., 1987), despite being previously used as term for Drosophila transformation (Fox \& Yoon, 1970) and for larger vesicles (average diameter: 500 to $1000 \mathrm{~nm}$ ) that shed from plasma membranes (Trams et al., 1981).

\footnotetext{
${ }^{1}$ The term exosome has also been widely used in RNA processing field for an eukaryotic complex containing 3'-5' exoribonucleases since its discovery in 1997 (Mitchell et al., 1997).
} 
Since then, exosomes, or exosome-like vesicles have been detected in the cultural medium of various animal cell types ${ }^{2}$, in particular of the hematopoietic system (Raposo et al., 1996, 1997; Zitvogel et al., 1998; Escola et al., 1998; Heijnen et al., 1999; Nguyen et al., 2003), but also of neurons (Tytell, 2005; Fauré et al., 2006), glial cells (Potolicchio et al., 2005; Fauré et al., 2006), epithelial cells (van Niel et al., 2001), and tumors (Wolfers et al., 2001). Exosomes are not only formed in cell culture, but also in vivo as they are detected in most body fluids such as plasma (Caby et al., 2005), milk (Admyre et al., 2007) and urine (Pisitkun et al., 2004). Cells of the hematopoietic system seem to use exosomes as signaling devices to shuttle molecules between antigen presenting cells. The role of exosomes in immunological processes has not only been important to understand how the immune system functions, but has also led to the development of exosome-based therapies. There are now several clinical trials that use exosomes as immunotherapeutical vehicles (Denzer et al., 2000). While a large number of studies have addressed in detail the composition and the potential physiological functions of exosomes, relartively little is known about the mechanisms of exosome biogenesis.

Based on their similar molecular composition and release pathways, the generation of retroviruses and exosomes have been regarded as related biological processes. In the so called Trojan exosome hypothesis it has been proposed that the retroviruses use the preexisting intra- and inter-cellular trafficking pathways of exosomes, implicating a possible mechanism of retroviral evolution (Gould et al., 2003).

\subsubsection{Structure and composition of exosomes}

Under electron microscope, exosomes appear cup-sphaped with a diameter of 30 to $100 \mathrm{~nm}$ (Théry et al., 2002b). Exosomes exhibit the same membrane topology as the plasma membrane: the cytosolic side of the lipid bilayer faces into the lumen while the extracellular side is exposed to the exterior (Simpson et al., 2008).

\footnotetext{
${ }^{2}$ It may be worth to mention that prior to the discovery of exosome release in mammalian systems, vesicles release from fusion of MVB had been proposed in the plants and fungi in 1967 and was investigated again recently(Marchant et al., 1967; Halperin \& Jensen, 1967; An et al., 2007). Recently, argosomes, exosome like membrane structures, were also found in Drosophila (Greco et al., 2001).
} 
The density of exosomes are generally between $1.13 \mathrm{~g} / \mathrm{ml}$ to $1.19 \mathrm{~g} / \mathrm{ml}$ in sucrose gradients $^{3}$ (Théry et al., 2002b). These typical features of exosomes allow the discrimination of exosomes from other membrane material. Apoptotic vesicles, for example, are much larger and have a density between $1.24 \mathrm{~g} / \mathrm{ml}$ to $1.28 \mathrm{~g} / \mathrm{ml}$ in sucrose gradients (Théry et al., 2001). However, sometimes exosomes are not easy to distinguish from small microvesicles that shed from the plasma membrane. A typical characteristic of exosomes is their unique molecular composition.

The protein composition of exosomes depend on the cell type they derive from, but there are a large number of proteins that are common from all cell types. The proteome analysis of exosomes from various types of cells and body fluids have identified several protein classes involved in different functions: integrins, Rab proteins, cytoskeleton elements, late endosomal/lysosomal proteins (LAMP-1, CD63), annexins, membrane receptors, heat shock proteins, enzymes etc. One of the most abundant and commonly found exosomal proteins are proteins of the tetraspanin family, such as CD9, CD63, CD81 and CD82. Tetraspanins are characterized by their four transmembrane domains and two extracellular domains that contain disulphide bridges. Apart of the common exosomal proteins there are also a number of proteins that are specific and depend on the cell type of origin. For example, MHC class II is an abundant protein of exosomes form antigen-presenting cells (Théry et al., 2002b; Simpson et al., 2008).

The lipid composition of exosomes has also been analyzed in a number of different cells. Immunoelectron microscopic labeling of cholesterol showed that cholesterol is enriched on exosomes from human lymphoblastoid cells (Möbius et al., 2002). This finding is consistent with mass spectrometry analysis of the lipid composition of exosomes derived from $\mathrm{B}$ lymphocytes (Wubbolts et al., 2003). In contrast, the content of cholesterol does not seem to differ between exosomes from reticulocytes and their plasma membrane (Vidal et al., 1989), nor between mast cell exosomes and their parent cells (Laulagnier et al., 2004). There are also similarities in the lipid composition between HIV and mast cell derived exosomes (Subra et al., 2007), supporting their common origin in some cells.

\footnotetext{
${ }^{3}$ Exosomes collected from circulation have a buoyant density of $1.21 \mathrm{~g} / \mathrm{ml}$ to $1.28 \mathrm{~g} / \mathrm{ml}$ (Caby et al., 2005)
} 


\subsubsection{Functions of exosomes}

\section{Molecule elimination}

The earliest function that was attributed to exosomes was their role in removing obsolete proteins. This was first observed in reticulocytes, which secrete the transferrin receptor in association with exosomes (Pan \& Johnstone, 1984). During the maturation of reticulocytes into erythrocytes, the levels of the transferrin receptor decreases in the plasma membrane as it is released together with exosomes (Pan \& Johnstone, 1983). A similar function of exosomes might be particular relevant for cells that are located towards a drainage system such as the gut or the kidney. Indeed, exosomes are found in relatively high levels in the urine and other body fluids (Pisitkun et al., 2004; Hoorn et al., 2005). To remove membrane with these body fluids might be simpler and energetically more favorable way as compared to the breakdown in the cellular degradation system.

\section{Intercellular signaling}

Antigen presenting cells (APCs), such as dendritic cells, macrophages, B lymphocytes and microglia, have the ability to internalize exogenous antigens and digest them into 15-22 residue peptides. These peptides can then be bound to major histocompatibility complex (MHC) class II molecules and presented together as peptide-MHC class II complexes on the cell surface. The APC displaying the processed exogenous peptides can activate $\mathrm{CD} 4^{+}$ T cells (Steinman, 1991).

A landmark finding in the exosome field was the discovery that B lymphocytes secrete exsomes with MHC class II in a peptide bound form (Raposo et al., 1996). This finding has spurred a number of follow-up studies on the the immunological functions of exosomes. Exosomes containing the peptide-MHC class II complexes derived from APCs can activate cognate or pre-activated $\mathrm{CD} 4^{+} \mathrm{T}$ cells. They can also activate naive $\mathrm{CD} 4^{+} \mathrm{T}$ cells when captured by dendritic cells. In this fashion, exosomes can transfer a pre-formed peptideMHC class II complex to another dendritic cells without the specific peptide-MHC class II complex and enable the cells to activate $\mathrm{CD} 4^{+} \mathrm{T}$ cells.

With the interaction of specific receptor-ligand interaction, the exosomes can be bound to the cell surface of a recipient cell. For example, exosomes bearing ICAM1 from mature 
dendritic cells can be captured by LFA1 on the surface of $\mathrm{CD} 8^{+}$dendritic cells and activate T cells (Segura et al., 2007). Recently, it has been suggested that phosphatidylserine within the extracellular leaflet of the exsosomal membrane of exosomes bind to TIM1 or TIM4 that are expressed on activated lymphocytes or phagocytes, respectively (Miyanishi et al., 2007). Another possibility is that MFGE8, which binds to both phosphatidylserine and integrins (Andersen et al., 1997), can bridge the phophatidylserine of exosomes to the integrins expressed on macrophages. Apart from their activating function, exosomes have also been shown to have inhibitory effects on immune cells. Exosomes from tumor are able to suppress the activity of lymphocyte, NK cell and CD8 ${ }^{+}$T cells (Liu et al., 2006; Clayton et al., 2007, 2008). Other studies have provided evidence that tumor derived exosomes can inhibit the proliferation and induce apoptosis of $\mathrm{T}$ cells (Abusamra et al., 2005).Exosomes purified from milk inhibit $\mathrm{T}$ cell activation and increase the number of regulatory cells in vitro (Admyre et al., 2007).

\section{Cargo shuttling}

Several studies have shown that exosomes can be found in various body fluids. One possibility is that they are simply removed from an organ or a tissue for degradation at another site. Another possibility is that their cargo is shuttled to cells at a more distant location where they might fulfill a specific function. Exosomes might, thus, serve as shuttles to exchange cargo between different cells in an organisms.

One interesting example are prion bearing exosomes. Prions are thought to be infectious proteins causing transmissible spongiform encephalopathies. The abnormally folded form of cellular prion protein, $\mathrm{PrP}^{\mathrm{sc}}$ (scrapie), catalyzes the transformation of normal cellular prion protein, $\mathrm{PrP}^{\mathrm{c}}$, to the abnormal, pathogenic $\mathrm{PrP}^{\mathrm{sc}}$ form (Prusiner, 1982). $\mathrm{PrP}^{\mathrm{c}}$ or $\mathrm{PrP}^{\mathrm{sc}}$ can be released into the medium in association with exosomes from many different cell types, such as cell lines expressing prion proteins (Fevrier et al., 2004), hematopoietic cells (Robertson et al., 2006; Griffiths et al., 2007) and cortical neurons and neuroblastoma cells (Fauré et al., 2006; Alais et al., 2008). Prion bearing exosomes can also be found in cerebral spinal fluid (Vella et al., 2008). Exosomes bearing $\operatorname{PrP}^{\mathrm{sc}}$ are infectious (Fevrier et al., 2004), and $\mathrm{PrP}^{\mathrm{sc}}$ containing exosomes derived from other cells are not only able to infect neuronal cell lines, but also to infect animals when these exosomes are injected intracerebrally (Vella et al., 2007). These observations suggest that exosomes might con- 
tribute to the intercellular transport and infectivity of $\mathrm{PrP}^{\mathrm{sc}}$.

Recently, it has been shown that exosomes can function as cargoes to transport mRNA and/or microRNA from one cell to another. Exosomes from mast cells contain functional mRNA and when transferred to a recipient cell proteins coded by the exosomal mRNA can be expressed (Valadi et al., 2007). Exosomes containing microRNA were also found in human blood and saliva (Hunter et al., 2008; Michael et al., 2009). The mechanisms of how exosomes fuse with the membrane of the recipient cell have not been resolved. However, if exosomes indeed shuttle mRNA from one cell to another, these vesicles have properties that qualify them as "endogenous virus" that allow a new mode of genetic exchange.

\subsubsection{Application of exosomes}

\section{Immunotherapies}

Soon after the discovery that exosomes are secreted by cells of the immune system, exosomes were proposed to be a new tool for therapy. Vaccination with dendritic cell derived exosomes preloaded with tumor peptides is one line of research that has been pursued in many studies. Dendritic cells treated with tumor peptides secrete exosomes which can prime tumor-specific cytotoxic $\mathrm{T}$ lymphocytes and promote rejection of tumor growth (Zitvogel et al., 1998). The induction of $\mathrm{CD}^{+} \mathrm{T}$ cell proliferation by antigen bearing exosomes requires dendritic cells bearing co-stimulatory molecules (Théry et al., 2002a). Exosomes bearing MHC class I - peptide complexes are functional with similar efficacy as mature dendritic cells for the priming of a $\mathrm{CD} 8^{+} \mathrm{T}$ cell response (André et al., 2004). To improve the therapeutic application of exosomes, a recent study used cyclophosphamide as a pre-treatment to inhibit regulatory $\mathrm{CD} 4^{+} \mathrm{T}$ cells and to enhance the anti-tumor effects of the therapeutic vaccines based on dendritic cell derived exosomes (Taieb et al., 2006).

Tumor cells are known to secrete exosomes in vivo and in vitro. Both immune stimulating and suppressing abilities of tumor derived exosomes have been reported. The $\mathrm{MHC}$ bearing exosomes from tumor can transfer tumor antigens and induce anti-tumor immunity (Wolfers et al., 2001; Andre et al., 2002; Altieri et al., 2004) . For example, exosomes derived from ovalbumin expressing EG7 tumor can stimulate the proliferation and differentiation of ovalbumin specific $\mathrm{CD} 8^{+} \mathrm{T}$ cells, although they are less affective than 
exosomes derived from ovalbumin pulsed dendritic cells (Hao et al., 2006). In contrast, tumor promoting effects of exosomes have also been observed. Pre-treatment of tumor derived exosomes can reduce the anti-tumor effect of vaccines of dendritic cell derived exosomes, probably through inhibition of nature killer cell cytotoxicity (Liu et al., 2006).

In addition, recent studies have shown that exosomes can induce angiogenesis and thus tumor invasiveness (Gesierich et al., 2006; Hood et al., 2009). Interestingly, exosomal secretion of tumor cells may be a pathway to export drugs, thereby increasing chemoresistance of these cells (Safaei et al., 2005) Hence, the pleiotropic effects of tumor exosomes and exosomal secretion may be used as therapeutic targets in cancer (Iero et al., 2008).

Phase I clinical trials using exosomes from autologous dendritic cells and ascites have been performed, showing tolerated toxicities and some beneficial responses (Escudier et al., 2005; Dai et al., 2008) Phase II/III trial using dendritic cell derived exosomes has also been proposed (Mignot et al., 2006).

\section{Biomarkers}

Because exosomes can be easily prepared from body fluids such as the peripheral circulation, urine and saliva, the microRNAs and protein patterns of exosomes might be used as diagnostic biomarkers for cancers or other diseases (Taylor \& Gercel-Taylor, 2008; Rabinowits et al., 2009; Michael et al., 2009).

\subsection{Multivesicular Bodies}

\subsubsection{Endosomal trafficking}

Endocytosis is a process of how cells communicate with the extracellular milieu, take up nutrients, turnover membrane and regulate signal transduction responsesi (Grant \& Donaldson, 2009; Gould \& Lippincott-Schwartz, 2009; Saftig \& Klumperman, 2009). The endocytic pathway consists of distinct compartments that internalize molecules from the plasma membrane and recycle them back to the surface via early endosomes and recycling endosomes or sort them for degradation using late endosomes and lysosomes. Early 


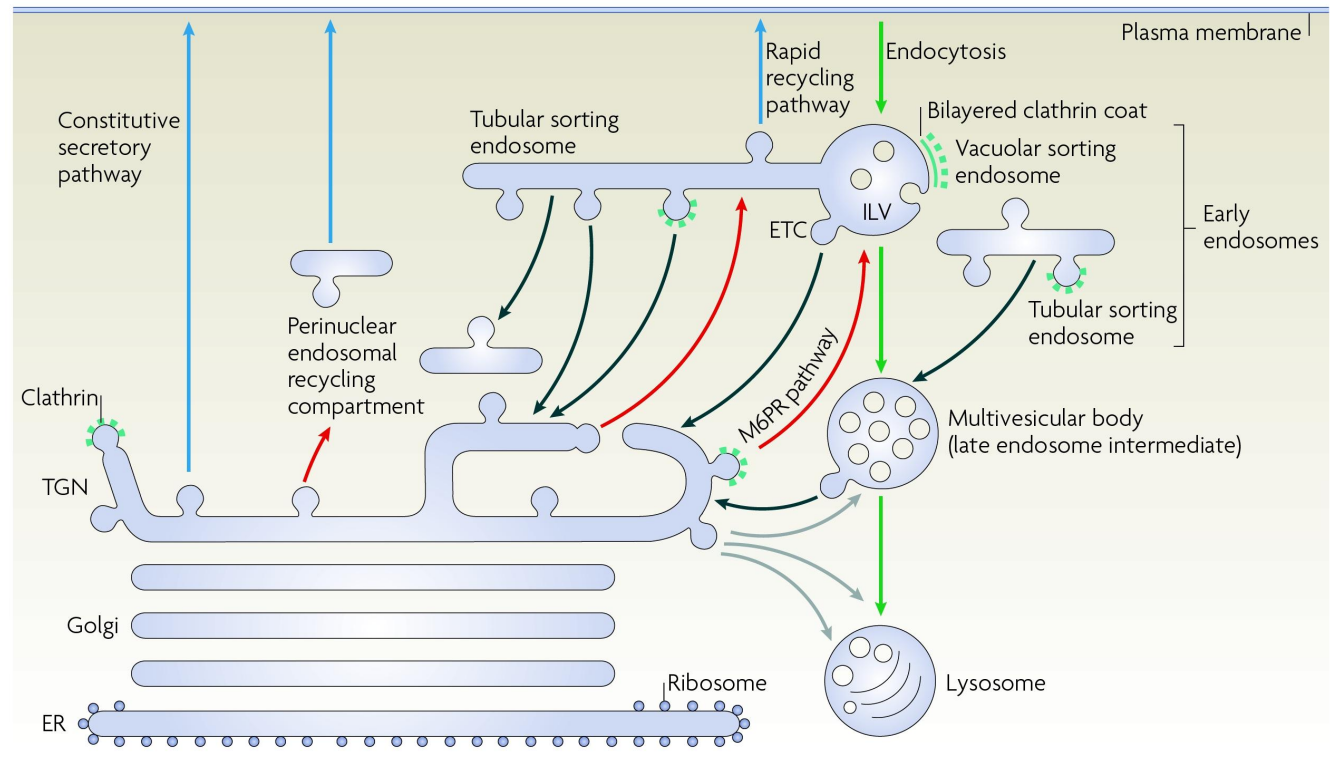

Figure 1.1: Pathways of endosomal trafficking. The main endocytic, cargo sorting pathways are conceptually summarized in the figure. Green arrows: endocytic pathway; blue arrows: constitutive secretory pathway; red arrows: direct intracellular pathway transporting cargoes such as the mannose-6-phosphate receptor (M6PR); grey arrows: cargoes enter the endo-lysosomal pathways at different stages; black arrows: retrograde pathways from endosomes. The exosome secretion pathway and lysosome secretion pathway (such as the lytic granule secretion) are not shown in the figure. For detailed discussion, see the main text in Section 1.2.1 and Section 1.2.2. ETC, endosome-to-TGN carrier; ILV, intraluminal vesicle. The figure is adapted from Saftig \& Klumperman (2009). Reprint by permission from Macmillan Publishers Ltd: Nature Reviews Molecular Cell Biology, copyright (2009)

endosomes are often located in the periphery of the cell and have a characteristic tubulovesicular morphology and a mildly acid $\mathrm{pH}$. Due to the acid $\mathrm{pH}$ many ligands dissociate from their receptors in early endosomes and are transported further into the endosomal system, whereas many of the receptors recycle back to the cell surface. Early endosomes deliver cargo to late endosomes using vesicular carriers such as MVBs and/or as part of their maturation process. Late endosomes are acidic (approx. $\mathrm{pH} 5.5$ ), contain ILVs, membrane lamellae and some proteins characteristic of lysosomes. Late endosomes can fuse with lysosomes to deliver material for the final digestion. Figure 1.1 illustrates a cartoonized summary of these processes. 
Much has been learned about endocytosis using the epidermal growth factor receptor (EGFR) as a model system. Ligand binding to the EGFR leads to its dimerization and autophosphorylation, followed by monoubiquitination of the activated EGFR at the plasma membrane. The degradation of EGFR is an important mechanim to terminate the signaling of the activated receptor at the plasma membrane. Hence, the process is a major negative feedback mechanism for receptor signaling regulation (Crosetto et al., 2005; von Zastrow \& Sorkin, 2007; Grewal \& Enrich, 2009; Sorkin \& Goh, 2009) .

Endocytosis of the EGFR is mediated by clathrin-coated pits (Huang et al., 2006). Immediately after its endocytosis the EGFR complex becomes concentrated within specific domains of the early endosomes where inward budding into the lumen is initiated. This step is required for the efficient downregulation of the EGFR signaling in the cytosol. Further transport through endosomal compartments involves multivesicular bodies and/or late endosomes to the lysosomes, the final destination of the degradative system (Futter et al., 1996). A small fraction of the EGFR is recycled back to the plasma membrane (Maxfield \& McGraw, 2004; Baldys \& Raymond, 2009). These different populations of endosomes are not only characterized by the time a endocytotosed molecule requires to reach an endosome, but also by its specfific biophysical properties. The intravesicular $\mathrm{pH}$ decreases from early $(\mathrm{pH} \sim 6)$ through late $(\mathrm{pH} \sim 5)$ endosomes and to the acidic lysosomes $(\mathrm{pH} 4.5-5)$ by the activity of the vacuolar $\mathrm{H}^{+}$ATPases. The components of endosomes also differ. For example, early endosomes contain Rab5 and early endosome antigen-1 (EEA1); late endosomes and multivesicular bodies are enriched in proteins such as LAMP1, LAMP2, LAMP3/CD63 and Rab7. Morphologically, early endosomes are vesicular and tubulovesicular and contain few ILVs $(<9)$, while late endosome and lysosome can be multivesicular or multilamellar (Tanaka et al., 2008; Saftig \& Klumperman, 2009).

Not all integral membrane receptors are degraded after ligand binding. The transferrin receptor, for example, is rapidly delivered back to the plasma membrane after its endocytosis. This process enables the cell to reuse the receptors for ligand binding and is thus referred to as receptor recyling (Grant \& Donaldson, 2009).

The transport between organelles is also mediated by endosomes. For example, mannose 6-phosphate receptor (M6PR) associates with some lysosomal proteins within the transGolgi network before being transported to late endosomes and lysosomes. After shuttling 
the cargoes, the M6PR dissociates from its cargoes and is delivered back to the trans-Golgi network (Ghosh et al., 2003; Braulke \& Bonifacino, 2009).

The separation of cargoes destined for degradation or recycling is mediated by the tubular sorting endosomes. They have the early endosome characteristics such as mildly acidic luminal environment. These endosomes consists of a vesicular and a tubular part. The cargoes that are transported for degradation remain in the vesicular part, whereas the membrane being recycled is sorted to the tubular compartment. These sorting endosomes do not only receive cargo from the plasma membrane but also from the trans-Golgi network. Therefore they act as a "hub" in intracellular membrane trafficking being able to sort membrane from different organelles to various destinations (Tooze \& Hollinshead, 1991; Stoorvogel et al., 1996; Carter \& Sorkin, 1998; Wilcke et al., 2000; Waguri et al., 2003; van Meel \& Klumperman, 2008; Saftig \& Klumperman, 2009).

\subsubsection{Late endosomes, lysosomes and lysosome related organelles}

During early endosomal maturation, the limiting membrane starts to bud into the lumen to form ILVs. These multivesicular structures are referred to as multivesicular bodies (MVBs), or multivesicular endosomes, and often considered as the intermediates of late endosomal compartments (Stoorvogel et al., 1991; Saftig \& Klumperman, 2009).

Sorting of late endosomal proteins from the biosynthetic pathways to the late endosomes as well as the homotypic and hetrotypic fusion of endosomes with other late endosomes and pre-existing lysosomes increase the degradative capacity in late endosomes(Mullock et al., 1998; Schmid et al., 1998; Antonin et al., 2000; Piper \& Luzio, 2001). Morphologically the lysosomes usually have globular shape, with electron-dense, often multilamellar lumen, however, they can also contain some ILVs. Because of the hetrogenesity in lysosomal morphology, lysosomes are usually defined operationally with their acidic luminal environment $(\mathrm{pH}<5)$, the appearance of LAMPs and the lack of MP6Rs. (Ohkuma \& Poole, 1978; Kornfeld \& Mellman, 1989)

A number of cell-type specific organelles have been characterized as lysosome related organelles, for example, the melanosomes from melanocytes, retinal cells and iris pigment epithelial cells; the lytic granules from cytotoxic T lymphocytes and NK cells; the platelet 
granules; the Weibel-Palade bodies in endothelial cells; the osteoclast granules in osteoclasts. Lysosome related organelles, late endosomes and lysosomes share many of their characteristics including the biogenesis pathways (Dell'Angelica et al., 2000; Raposo \& Marks, 2002; Raposo et al., 2007).

Melasomes are specialized to synthesize and to store pigment, melanins, which determine the skin and hair color as well as contribute to the protection from ultraviolet radiation. The key marker protein of melanosomes is Pmel17 (Berson et al., 2001). The protomer of Pmel17 is synthesized in the endoplasmic reticulum (ER), modified with oligosaccharides in the Golgi, and transported into early/sorting endosomes (Harper et al., 2008). The Pmel17 protomer within the limiting membrane of the endosome is then sorted into the lumen by its inward invagination and subsequent formation of ILVs. This process is not dependent on ubiquitination of the protein within its cytosolic domain, but relies solely on the luminal domain of Pmel17 (Berson et al., 2001). When Pmel17 is sorted into the lumen it is cleaved and then starts to polymerize (Berson et al., 2003). Finally, the organelle containing polymerized Pmel17 recruits more melanosomal cargoes such as the melanogenic enzymes and other factors for movement to the cell periphery. The last step includes the transfer of the mature melanosomes to keratinocytes (Wu et al., 2001; Seabra \& Coudrier, 2004).

Unlike melanosomes, which co-exist with lysosomes in the same cells (Smit et al., 1993), some lysosome related organelles are considered as specialized lysosomes and cells with these organelles contain no normal lysosomes (Dell'Angelica et al., 2000). Another group of lysosome related organelles are secretory lysosomes ${ }^{4}$. Secretory lysosomes play especially important roles in the immune system. For example, the lytic granules from the cytotoxic $\mathrm{T}$ lymphocytes and nature killer cells contain perforin, a pore forming protein which creates pores in the plasma membrane of target cells by its self polymerization (Podack et al., 1985; Masson \& Tschopp, 1985). Upon stimulation such as receptor-antigen recognition (immunological synapse), the lytic granules are moved to the cell periphery in a microtubule dependent manner and its content is secreted into the extracellular space (Stinchcombe et al., 2001; Burkhardt et al., 1993; Wei et al., 1998). Granzymes from the

\footnotetext{
${ }^{4}$ The term "secretory lysosomes" sometimes is referred to as all the lysosomal related organelles (e.g. Holt et al. (2006)). In this work, it is emphasized to distinguish the conventional lysosomes and other lysosome related organelles which can be found together with other conventional lysosomes.
} 
lytic granules can then pass through the pores formed by perforin and trigger the rapid apoptosis of the pathogen infected cells (Pasternack et al., 1991; Shi et al., 1992).

An interesting aspect of the lysosome related organelles is their movement to the cell periphery and fusion with plasma membrane. One central protein in this process is the Rab GTPase (discussed in section 1.4), Rab27a. Rab27a recruits effectors such as the Synaptotagmin-like protein family, e.g. Slp2a and melanophilin, in a GTP dependent manner (Fukuda, 2002; Kuroda et al., 2002; Fukuda, 2006). Melanophilin and Slp2a are required for the movement of melanosomes to the plasma membrane (Strom et al., 2002; Kuroda \& Fukuda, 2004). Rab27a also binds to Munc13-4 in a GTP dependent manner (Goishi et al., 2004; Shirakawa et al., 2004). Mutation of Munc13-4 results in a severely reduced ability for cytotoxic $\mathrm{T}$ lymphocytes to kill the target cells, caused by the deficiency of secretion of lytic granule content, but not cytokines. It has been suggested that Munc13-4 functions to prime the machinery required for lytic granule fusion (Feldmann et al., 2003; Neeft et al., 2005).

\subsubsection{Biogenesis of intraluminal vesicles I - the ESCRT machinery}

The best studied machinery of ILV formation in multivesicular bodies is the endosomal sorting complex for transport (ESCRT) machinery. The components of the ESCRTs are

highly conserved in eukaryotes (Leung et al., 2008; Ghazi-Tabatabai et al., 2009). The ESCRT machinery is known for the degradation of ubiquitylated membrane proteins. In yeasts (Saccharomyces cerevisiae), proteins forming the ESCRTs were identified as the vacuolar protein sorting (vps) mutants (Raiborg \& Stenmark, 2009). After receptorligand interaction, signaling receptors, such as epidermal growth factor receptor (EGFR), become ubiquitylated and subsequently delivered to the lysosome for attenuation of the signal (Sorkin \& Goh, 2009). After multiple monoubiquitylation or Lys-63-linked polyubiqutilation, a membrane protein is recognized by the ESCRTs and transported into the ILVs (Katzmann et al., 2001; Alam et al., 2004; Barriere et al., 2007; Lauwers et al., 2009). 
The ESCRTs machinery consists 4 major complexes, ESCRT-0 ${ }^{5}$, -I, -II, and -III, together with other components, such as Alix and the VPS4 AAA-ATPase complex (Figure 1.2). The two proteins of ESCRT-0 are Hrs and STAM, each of which contains the ubiquitin and clathrin binding VHS (Vps27, Hrs and STAM) domain (Hofmann \& Falquet, 2001; Raiborg et al., 2001; McCullough et al., 2006). The FYVE domain of Hrs mediates the recruitment of the ESCRT-0 to the endosomal membrane though phosphatidylinositol 3phosphate (PtdIns(3)P) (Gaullier et al., 1998; Komada \& Soriano, 1999). Hrs also bridges ESCRT-0 and ESCRT-I through its binding to the UEV domain of Tsg101, an ESCRT-I component (Pornillos et al., 2003). The ESCRT-I is an 18-25 nm elongated heterotetrameric complex containing one copy of Tsg101, Mvb12, Vps37 and Vps28 (Kostelansky et al., 2007). ESCRT-0 is required for the recruitment of ESCRT-I to endosomal membranes (Bache et al., 2003). ESCRT-II is also a heterotetramer containing one Vps22, one Vps36, and two Vps25 molecules (Hierro et al., 2004). ESCRT-I is linked to ESCRT-II through the interaction of Vps28 with the GLUE domain of Vps36, which has the capacity to bind phosphatidylinositol $(3,4,5)$-triphosphate $\left(\operatorname{Ptd} \operatorname{Ins}(3,4,5) \mathrm{P}_{3}\right)$ and ubiquitin (Slagsvold et al., 2005; Teo et al., 2006).

Unlike ESCRT-0, ESCRT-I and ESCRT-II, which form stable, defined hetero-oligomeric complexes on the membranes upon cargo sorting, the ESCRT-III proteins, Vps20, Snf7 (Vps32), Vps24, and Vps2 (yeast nomenclature; for the metazoan synonyms, see Williams \& Urbé (2007)), oligomerize in a membrane dependent, higher-ordered fashion. The initiation of ESCRT-II polymerization is the recruitment of Vps20 to Vps25 in ESCRT-II, triggering the filamentous oligomerization of Snf7. The Snf7 oligomers were reported to be capped with Vps24, which interacts with Vps2. Through Vps2, the association of Vps4 initiates the disassembly of the ESCRT-III complex, which is the only energy consuming step directly linked to the ESCRT machinery (Teis et al., 2008; Saksena et al., 2009).

Overexpression of Snf7, the oligomerizing component of ESCRT-III, induces tubules from the plasma membrane, triggered by the formation of spiral filament of Snf7 (Hanson et al., 2008). In vitro, ESCRT-III components, CHMP2A and CHMP3, can form a helical tubules which expose the membrane associating domains to the out-side (Lata et al., 2008). These

\footnotetext{
${ }^{5}$ The Hrs-STAM complex was not originally classified as an ESCRT complex, but was later proposed to be termed as ESCRT-0 for their functional and biochemical association to the ESCRT-I (Williams \& Urbé, 2007).
} 


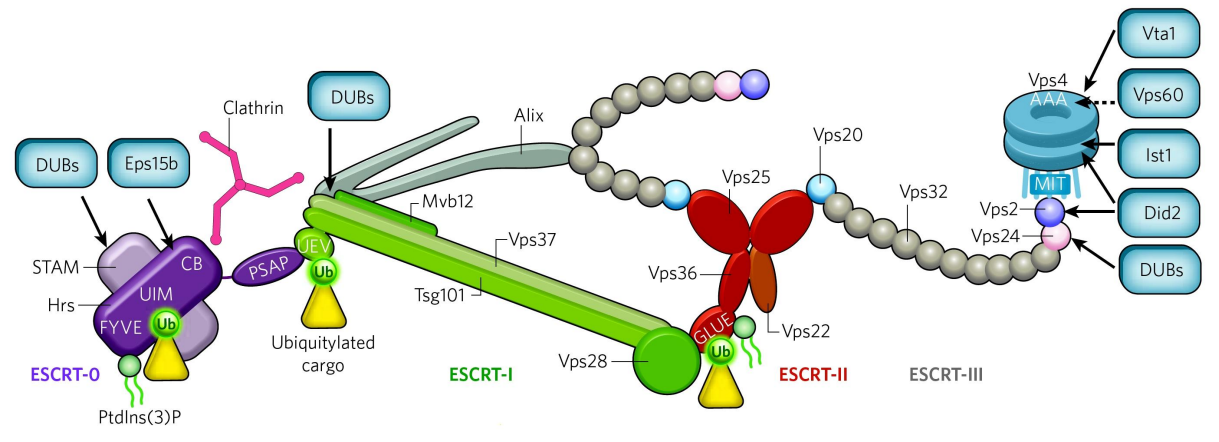

Figure 1.2: Composition and molecular interactions of the ESCRT machinery. The constituents and interactions of ESCRTs and the associated components, deubiquitylating enzymes (DUBs), Alix and the Vps4 AAA-ATPase, are illustrated. Protein domains are labelled in white. $\mathrm{CB}$, clathrin-box motif; UB, ubiquitin; dashed arrow, predicted interaction. The figure is adapted from Raiborg \& Stenmark (2009). Reprint by permission from Macmillan Publishers Ltd: Nature, copyright (2009)

findings support the view that the structural assembly of ESCRT-III is the driving fource of initiating membrane curvature, in a topological manner consistent with the budding of ILVs into MVBs.

The mechanism of vesicular budding from the plasma membrane into the cytosol is well known for clathrin-mediated endocytosis. Here, the vesicles are formed in an opposite directions as compared to ILV formation. The final fission of endocytic vesicles is driven by the GTPase dynamin through a GTPase activity dependent conformational change (Sweitzer \& Hinshaw, 1998). There are only few examples of vesicle formation that occur in topological manner similar to ILV formation. Viral budding from the plasma membrane toward the extracellular space is one well studied example or cytokinesis, which involves the formation of the midbody ring that requires the protruding curvature of the plasma membrane is another example. ESCRTs are known to be involved in all of these types of membrane abscission (Martin-Serrano et al., 2003; Spitzer et al., 2006; Carlton \& MartinSerrano, 2007; Raiborg \& Stenmark, 2009). ILV formation, viral budding, and cytokinesis have in common that they require the involvement of ESCRT-I and ESCRT-III (Pincetic et al., 2008; Carlton et al., 2008). The mechanism of viral budding will be discussed in more details later. 


\subsubsection{Biogenesis of intraluminal vesicles II - lipid dependent machanisms}

In most cases the formation of ILVs in MVBs is mediated by the ESCRT machinery. However, this is not the only mechanism for ILVs biogenesis. Depletion of components of the ESCRTs results in formation of class E compartments in yeast (Hurley \& Emr, 2006) and causes enlargement of endosomes in mammalian cells. Cells depleted of Tsg101 results in the formation of endosomes with reduced number of ILVs, but ILVs are still present in a relatively large number of the endosomes. Knockdown of Tsg101 also inhibits the formation of MVBs after EGF stimulation (Razi \& Futter, 2006). Depletion of Hrs, however, reduces the number ILVs and induces formation of large vacuoles; EGF is still be found in these MVBs with very few ILVs (Razi \& Futter, 2006; Pons et al., 2008). After knocking down Vps22, a component of ESCRT-II, the transport of EGFR into the lumen of MVBs is reduced, without altering the mophology of the MVBs dramatically (Malerød et al., 2007). The phenotype is similar to that of cells lacking Vps24, a subunit of ESCRT-III, which contains smaller MVBs than control cells (Bache et al., 2006). After knockdown of one component of each of the ESCRT-0, ESCRT-I, ESCRT-II and ESCRT-III, the EGF induced formation of MVBs is highly reduced. However, MVBs still form in an EGF independent manner (Stuffers et al., 2009). These studies strongly implicate the existence of an ESCRT-independent machinery of ILV formation.

In order to generate ILVs, a huge negative membrane curvature has to be induced on the limiting membrane of the endosome. In addition to the protein-based mechansims, which impose a curvature change by forces induced by ESCRT-III polymers, lipids can contribute to membrane curvature changes (McMahon \& Gallop, 2005).

For example, phosphatidic acid and lysophosphatidic acid, which have spontaneous negative and positive curvatures, respectively (Kooijman et al., 2005), can be formed and introduce membrane curvature by the activity of lysophosphatidic acid acyl transferase and phospholipase $\mathrm{A}_{2}$ (Leung, 2001; Brown et al., 2003). After changing the expression level of lysophosphatidic acid specific acyltransferase 3, a morphology change is induced upon the Golgi apparatus (Schmidt \& Brown, 2009).

In addition, lysobisphosphatidic acid (LBPA, or bis(monoacylglycero) phosphate, BMP), induces the formation of multivesicular liposomes in vitro in low $\mathrm{pH}$. Alix, an ESCRT associated protein, is recruited by the LBPA containing liposomes and is able to inhibit 
the formation of ILVs in these liposomes. The LBPA-based intraluminal invagination of endosomes in vivo is also controlled by Alix (Matsuo et al., 2004). Interestingly, LBPA and Alix are also required for the fusion of vesicular stomatitis virus (VSV) with the late endosomal membrane, a critical step for virus infection (Blanc et al., 2005). In addition, Alix controls the budding of vesicles from acidic late endosomal membrane in vitro (Falguières et al., 2008). Alix is, thus, a protein that may connect a lipid- to a protein-based machinery as Alix is also known to associate with ESCRT components.

\subsection{Biogenesis of exosomes}

\subsubsection{Retrovirus budding}

The topology of the retroviral lipid membrane is similar to that of an exosome - the cytosolic side faces the inside. To form a viral membrane, retroviruses do not only use the cellular membrane, but also the pre-existing ESCRT machinery for membrane budding (Hurley \& Emr, 2006; Carlton \& Martin-Serrano, 2009; Usami et al., 2009; Pincetic \& Leis, 2009).

Human immunodeficiency virus (HIV) is a retrovirus with a spherical shape with a diameter of $\sim 120 \mathrm{~nm}$. It contains two copies of positive single stranded genomic RNA and reverse transcriptase that are enclosed by a conical shaped capsid. The capsid is surrounded by a matrix composed of the viral protein, p17, and the outermost envelope consists of the lipid bilayer and glycoproteins (Gelderblom et al., 1989). The HIV type 1 (HIV-1) gag gene encodes the Gag proteins, which are initially synthesized as a polyprotein precurser, Pr55 Gag. During or after viral budding, the Pr55 Gag is cleaved into mature structural proteins such as the p17 matrix, the p24 capsid, the p7 nucleocapsid, and p6. Interestingly, expression of the Pr55 Gag (Gag) is sufficient to form non-infectious, virus-like particles (VLPs) without other viral proteins or viral RNA (Freed, 1998). Gag proteins from other retroviruses, such as murine leukemia virus, also have ability to form VLP. Moreover, the VLPs formed by Gag are still able to recruit similar amount of RNA as the original virions (Muriaux et al., 2001; Rulli et al., 2007).

VLP formation by the Gag Protein is regulated by different domains, including the membrane targeting domain, the Gag-Gag interaction domain and the C-terminal p6 domain. 
The HIV-1 p6 domain is necessary for the recruitment of an ATP-requiring cellular machinery for the separation of virion from the cellular membrane (Tritel \& Resh, 2001). There are two smaller domains, the late domains, on the HIV-1 p6, consisting of PTAP (Pro-Thr-Ala-Pro) motif and aother domain with the LYPX ${ }_{n} L$ (Leu-Tyr-Pro-Xaa - Leu) motif. The UEV domain of Tsg101 can form a complex with PTAP peptide of HIV-1 p6 (VerPlank et al., 2001; Pornillos et al., 2002) and the UEV-PTAP interaction is important for the VLP formation as well as virus assembly (Garrus et al., 2001; Usami et al., 2008). The $\mathrm{LYPX}_{\mathrm{n}} \mathrm{L}$ motif and the nucleocapsid domain contribute to the interaction between Gag and Bro1 domain of Alix (Strack et al., 2003; Fisher et al., 2007; Lee et al., 2007; Popov et al., 2008) and the interaction seems to be required for virus release in an ESCRT-III dependent mechanism (Zhai et al., 2008).

\subsubsection{Biogenesis of exosomes}

Exosomes are generally considered to originate as ILVs within MVBs and to be released after fusion of the MVBs with the plasma membrane. Therefore, the formation of ILVs in MVB is the key step of exosome biogenesis. Although the ESCRT machinery is the most widely studied mechanism for the biogensis of ILVs, lipid-based mechanisms may also exist.

These lipid-based mechanisms might be relevant for exosome biogenesis as they seem to be enriched in raft-lipids such as cholesterol and sphingolipids (de Gassart et al., 2003). In addition, a study on the "endosome-like domains" of the plasma membrane of Jurkat $\mathrm{T}$ cells, that are recognized as HIV budding site, shows that the high-order oligomerization of cargo is sufficient for the sorting into exosomes or VLPs. The sorting and the formation of the vesicle occurred independently of the ESCRT machinery (Fang et al., 2007). Furthermore, antibody induced aggregation of transferrin receptor on reticulocytes and peptide-MHC-II complexes on B cells increases the sorting or the clustered proteins into exosomes (Vidal et al., 1997; Muntasell et al., 2007). However, the molecular details of the machinery required for the sorting of cargo into exosomes has not been elucidated.

Another poorly understood process is the fusion of MVBs with the plasma membrane. It has been hypothesized that the mechanism is similar to the calcium-dependent fusion of secretory lysosomes (van Niel et al., 2006; Andrews \& Chakrabarti, 2005). Indeed, in many cell types calcium ionophore are able to trigger the secretion of exosomes (Savina 
et al., 2003; Fauré et al., 2006; Valadi et al., 2007; Krämer-Albers et al., 2007). VAMP7, a v-SNARE, which involves in lysosome secretion, was also found to play a role in MVB and plasma membrane fusion (Fader et al., 2009). Recently a study showed that Rab27a and Rab27b control the exosomal secretion pathway through their function in MVB docking at the plasma membrane. Interestingly, knock-down of Slp4, a Rab27a effector, resulted in a similar phenotype as the knockdown of Rab27a (Ostrowski et al., 2009). Slp4 is known to mediate the release of dense-core vesicles by promoting the docking of these vesicles to the plasma membrane (Fukuda et al., 2002; Fukuda, 2006). These results imply that the exosome release pathway and regulated exocytosis have mechanisms in common.

An earlier study has shown that exosome release is regulated by Rab11 in a GTPdependent manner (Savina et al., 2005), suggesting that exosome formation share mechanisms with the receptor recycling pathway.

\subsection{The Rab GTPases}

\subsubsection{Rabs in intracellular trafficking}

Intracellular membrane trafficking in the cell is highly regulated and coordinated process that not involves the transportation of membrane within the cell, but also the control of membrane budding and fusion. To ensure the proper directionality of these cargo bearing organelles, the identity of a certain vesicle has to be marked in order to be recognized by intracellular receptors at a specific site within the cells . The Rab protein family fulfill some of these functions. They are part of the superfamily of Ras like small guanine triphosphatases (GTPases) and exist either in an "active" GTP-bound form or in an "inactive" GDP-bound state. Rabs are known to play an important role in coordinating intracellular membrane trafficking. More than 60 Rab proteins have been identified with a wide spectrum of intracellular trafficking functions and subcellular localizations as summarized in Figure 1.3 (Stenmark, 2009). Known functions of the Rabs include the regulation of vesicle budding, uncoating, mobility and fusion.

Despite being considered as GTPases, Rabs, like other small GTPases, such as Ras, Rho or Ran families, are incomplete enzymes. Only after the binding of a specific GTP-hydrolysis activating protein (GAP), a Rab can efficiently hydrolyze the GTP molecule it carries. 


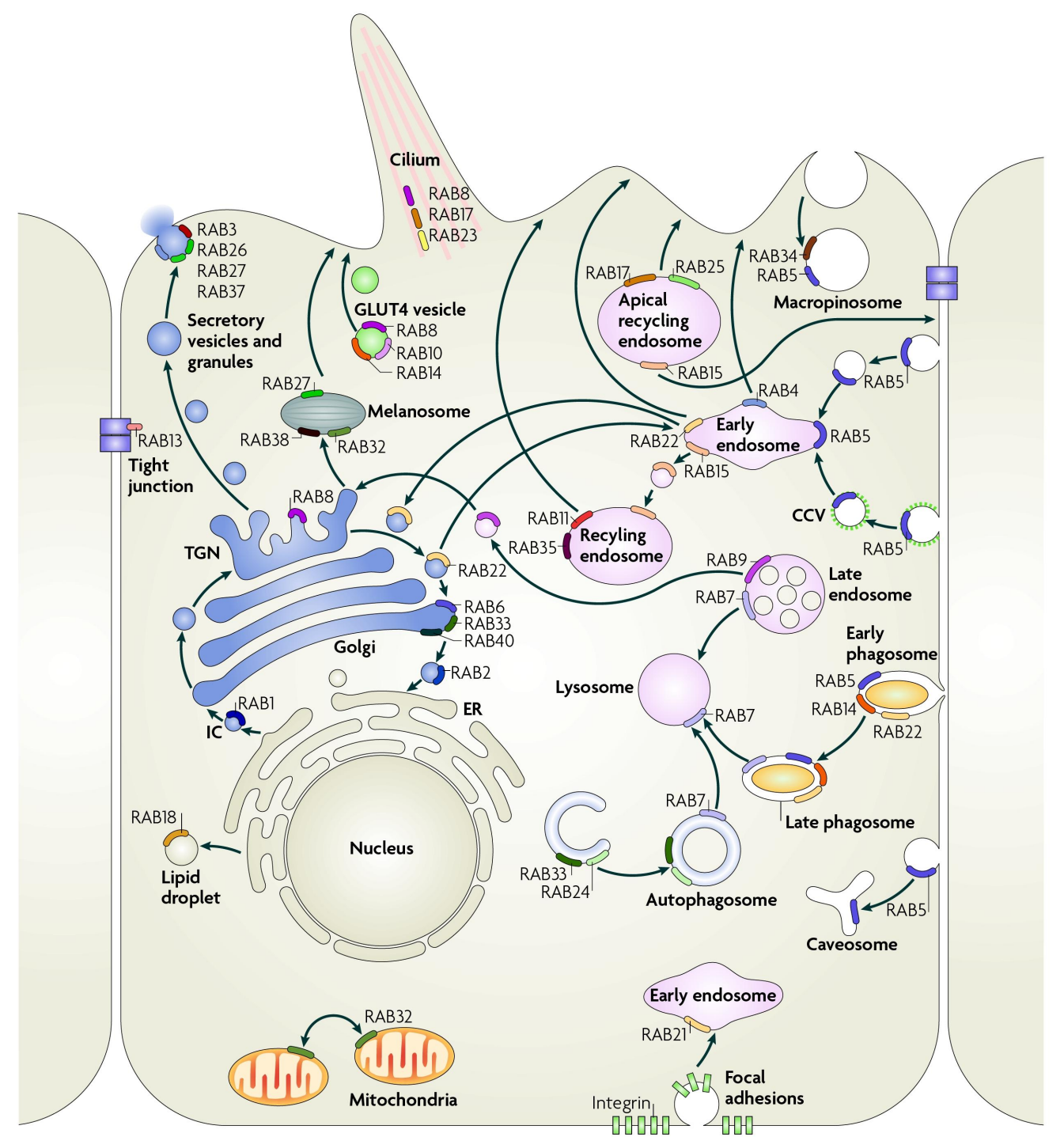

Figure 1.3: Localization and function of Rab GTPases. Selected Rab GTPases are shown in a conceptual epithelium cell to illustrate the current understanding of their intracellular localization and function. The functions of Rabs on intra-endosomal trafficking and the mechanisms of Rab domains on endosomal identidies are emphasized. For detailed discussion, see Section 1.4. The figure is adapted from Stenmark (2009). Reprint by permission from macmillan publishers Itd: nature reviews molecular cell biology, copyright (2009). 
Rab GAPs have a conserved TBC (Tre2, Bub2 and Cdc16) domain, with two conserved Arg and Gln residues within the catalytic center (Pan et al., 2006). The conversion of the GTP-bound state to the GDP-bound state of a Rab protein is therefore mediated by its GAP. A Rab in the GDP-bound form is bound to a GDP dissociation inhibitor (GDI), which not only protects the dissociation of the GDP molecular (Matsui et al., 1990), but also hids the hydrophobic prenyl anchor of the Rab (Ullrich et al., 1993, 1994; Soldati et al., 1994). For a Rab to anchor into a membrane, a membrane bound GDF (GDI displacement factor) has to replace the GDI (Pfeffer \& Aivazian, 2004). The exchange of GDP to GTP of Rabs is mediated by guanin nucleotide exchange factors (GEFs). The binding of a GEF to a Rab facilitates the release of GDP and the nucleotide empty Rab recruits a GTP immediately due to the high concentration of GTP $(\sim 1 \mathrm{mM})$ in the cytosol (Stenmark, 2009). The Rab switch therefore includes the exchage of the GDP- to the GTP-bound state mediated by GEFs and the exchange of the GTP- to the GDP- bound state by GAPs.

The GTP bound forms are generally regarded as an active state of a Rab protein, which recruits specific effectors. There are very few Rab effectors that prefer the GDP bound form, e.g. the binding of protrudin to Rab11-GDP (Shirane \& Nakayama, 2006). The structural studies on Rabs reveal the basis of the molecular switch mechanisms. Rab proteins, like Ras small GTPases, have two switch elements, which are able to change the conformation upon nucleotide binding (Dumas et al., 1999; Pereira-Leal \& Seabra, 2000; Garcia-Saez et al., 2006). Hence the switch regions play an important role in effector binding and thus in the allocation of Rab function. For example, a mutation in the switch I region of Rab27a results a loss of its binding ability to melanophilin, thereby inducing the perinuclear localization of melanosome in melanocytes (Westbroek et al., 2008).

Studies have shown that many Rabs have specific localization on distinct organelle (Figure 1.3). This character together with their molecule switch property implicate that they play roles in organelle identity. For example, Rab5 is found specifically localized on early endosomes (Chavrier et al., 1991). With low level of expression of GFP-tagged Rabs, it has been shown that there are even separate Rab-domains within a membrane. Rab4, Rab5 and Rab11 are observed on distinct domains on recycling endosomes, whereas Rab7 and Rab9 localize in late endosomal domains (Sönnichsen et al., 2000; Barbero et al., 2002). In addition, the time that different Rab proteins remain on an organelle or a vesicle can differ. With high speed imaging and vesicle tracking algorithms, individual Rab5 
containing early endosome were shown to move towards the cell center thereby losing its Rab5 signal and gaining the Rab7 signal (Rink et al., 2005). This study has established the concept that the maturation of endosomes is mediated by Rab conversion.

\subsubsection{Rabs in tethering factor recruitment}

Rab proteins mediate intracellular membrane transport not only by establishing membrane identity of a specific organelle but also by their ability to recruit effectors. One group of the Rab effectors functions in vesicle tethering.

For membrane transport to occur, the vesicular and the target membrane has to fuse, overcoming the electrostatic force between the negatively charged lipid bilayer surfaces. Fusion is mediated by the protein family SNAREs ( $N$-ehtylmaleimide-sensitive factor attachment protein receptors). The formation of a fusogenic SNARE complex occurs by the assembly of one $\alpha$-helix from the vesicle (v-SNARE) and three from the target membrane (t-SNAREs) that bring the membrane to close proximity and in order to facilitate fusion (Jahn \& Scheller, 2006). Due to their compartment specific localization, SNARE proteins can contribute to the pairing of membranes in intracellular transport (Pelham, 2001). However, it has also been shown that in addition to SNAREs, tethering factors are required for membrane pairing. These factors mediate the membrane recognition step and bring the membrane together before the SNARE complex is formed (Sztul \& Lupashin, 2006). A model depicting the tethering process is shown in Figure 1.4.

There are two general types of tethering factors, the coiled-coil tethers and the multisubunit tethering complexes. Both types have been found to be regulated by Rab proteins. For example, EEA1, is a Rab5 effector and a coiled-coil tethering factor that is required for early endosome attachment and its interaction with Syntaxin 13, a t-SNARE, is necessary to drive homotypic endosome fusion (Christoforidis et al., 1999; McBride et al., 1999). In yeast, Sec15p, a component of exocyst, which is a multimeric tethering complex required for exocytosis within the secretory pathway, is a effector of a Rab GTPase, Sec4p (Guo et al., 1999). In the mammalian system, Sec15 in the exocyst is an effector of Rab11 (Zhang et al., 2004). These studies illustrate that the Rab proteins can regulate membrane fusion by controlling SNARE machinery indirectly through a conserved interaction 


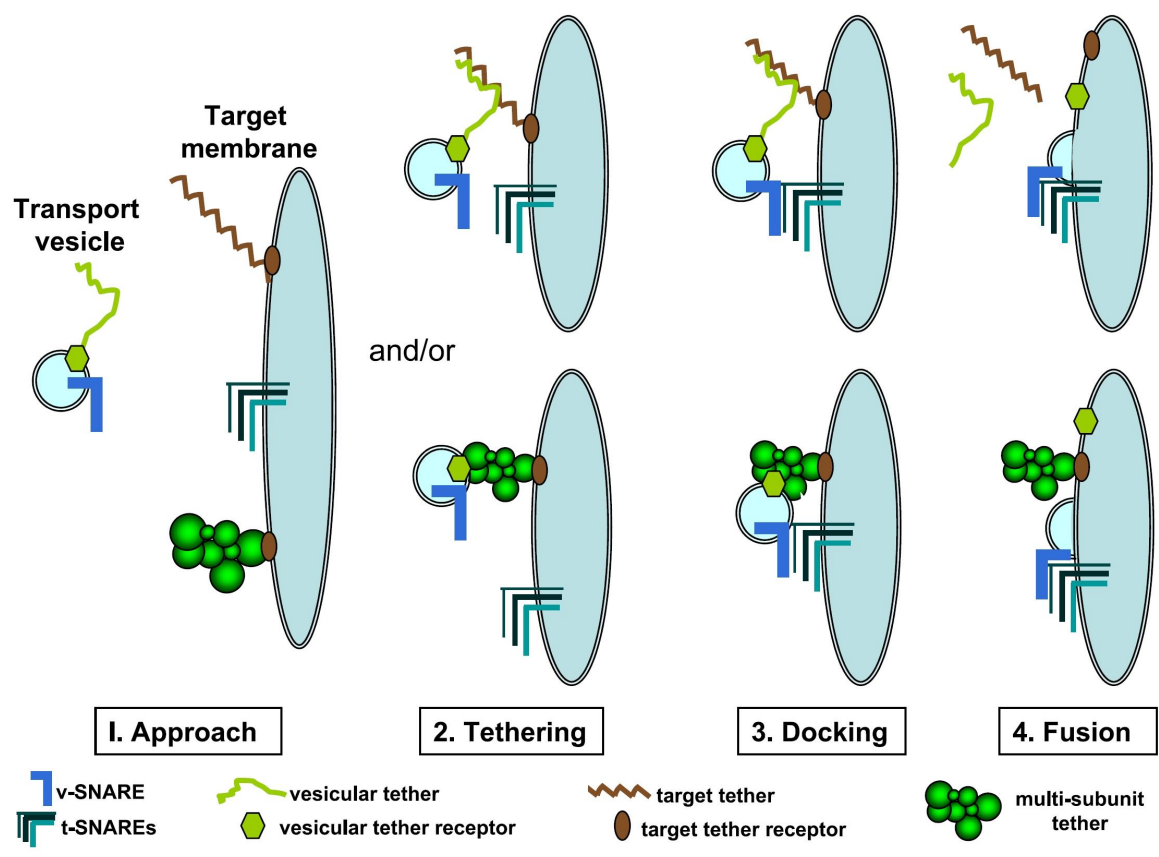

Figure 1.4: A model for tethering during membrane traffic. As the vesicle moved by diffusion or motor mi dated transport to a region close to the targeted membrane (>200 nm) (1. Approaching), the tethers on the vesicle and the membrane interact each other and the vesicle is tethered to the membrane (2. Tethering). Later, SNAREs on the vesicles and the membrane form transSNARE complexes (3. Docking) and the energy of the final membrane fusion step is provided by the complete formation of the SNARE complexes (4. Fusion). The figure is adapted from Sztul \& Lupashin (2006), used with permission. 
between the different tethering factors.

\subsection{The Proteolipid Protein}

The myelin sheath is a multilamellar structure surrounding axons, providing the insulation that facilitates the saltatory conduction of the electrical axonal signal transmission. In the central nervous system (CNS), the myelin sheath is produced by oligodendrocytes, that extend a large number of cellular processes, of which each can wrap different axons and form several myelinated segments. Chemically, myelin is composed of $70 \%$ of lipids and $30 \%$ of proteins by dry weight. Due to the high proportion of lipids myelin can easily be purified from other cellular membrane due to its low density (Baumann \& Pham-Dinh, 2001). Myelin is enriched in cholesterol and the glycosphingolipids, galactosylceramides and sulfatides. Among the proteins identified in CNS myelin, proteolipid protein (PLP, $\sim 50 \%$ ) and myelin basic protein (MBP, $\sim 30 \%$ ) are the most abundant ones.

PLP is a low molecular weight protein composed of 276 amino acids that transverse the membrane 4 times with the $\mathrm{N}$ - and $\mathrm{C}$ - terminal facing the cytoplasm. Alternatively spliced PLP mRNA produces DM-20, which differs from PLP by a 35 amino acid segment deletion in the intracellular loop (Nave et al., 1987). Mice with targeted deletions of PLP still form myelin, although in a less stable form (Klugmann et al., 1997). However, the major phenotype of mice lacking PLP is a late-onset neurodegeneration (Griffiths et al., 1995).

PLP is synthesized in the ER, transported through the Golgi and targeted to the plasma membrane. Mutations of PLP which lead to PLP accumulation in the ER, such as the jimpy-msd mouse mutant, result in a severe dysmyelinating phenotype caused by the induction of apoptosis of oligodendrocytes at the late stage of maturation (Inoue, 2005). Recently, it is found that in Oli-neu cells, the immortalized murine oligodedroglial precursor cells (Jung et al., 1995), exogenously expressed PLP is transported to the plasma membrane and endocytosed by a cholesterol-dependent mechanism. PLP accumulates in the late endosomal or lysosomal compartment and the movement of these PLP-containing endosomes back to plasma membrane is triggered after the differentiation of the cells (Trajkovic et al., 2006). 


\subsection{Goal of the project}

The goal of this project was to elucidate the molecular mechanisms of exosome biogenesis. As a model system, Oli-neu cells expressing PLP were used. The starting point of the first part of the thesis was the question whether the ESCRT machinery is involved in exosome biogenesis. We find that exsosome biogenesis is ESCRT-independent and define a new mechanism of how a vesicle is formed.

In the second part, we aimed at identifying the RabGTPases required for exosome generation. We identify Rab35 as new Rab that controls MVB trafficking. 


\title{
Chapter 2
}

\section{Materials and Methods}

\subsection{Materials}

\subsubsection{Chemicals and comsumables}

All the chemicals were purchased from Sigma-Aldrich, Merck or AppliChem, unless specified. The comsumables for biochemical reactions and cell culture were purchased from Eppendorf and Falcon.

\subsubsection{Cell lines}

\author{
Name Origin \\ Oli-neu J. Trotter, University of Mainz, Mainz, Germany \\ PC-3 P. Burfeind, University of Göttingen, Göttingen, Germany \\ DH5 $\alpha$ Invitrogen
}




\subsubsection{Antibodies and other proteins}

\section{Primary antibodies}

$\begin{array}{lll}\text { Target } & \text { Host species } & \text { Origin } \\ \text { Actin (AC-40) } & \text { Mouse } & \text { Sigma-Aldrich } \\ \text { Contactin }(\text { K67/25) } & \text { Mouse } & \text { NeuroMab } \\ \text { Flotillin-1 } & \text { Mouse } & \text { BD Bioscience } \\ \text { Flotillin-2 } & \text { Mouse } & \text { BD Bioscience } \\ \text { GFAP } & \text { Rabbit } & \text { Promega } \\ \text { GFP } & \text { Rabbit } & \text { Abcam } \\ \text { GFP } & \text { Rabbit } & \text { Invitrogen } \\ \text { Synaptophysin } & \text { Mouse } & \text { Synaptic Systems } \\ \text { HA-tag (16B12) } & \text { Mouse } & \text { Convance } \\ \text { Lamp-1 (CD107a) } & \text { Rat } & \text { BD Bioscience } \\ \text { myc-tag } & \text { Mouse } & \text { Cell Signalling } \\ \text { myc-tag } & \text { Rabbit } & \text { Upstate } \\ \text { PLP (3F4) } & \text { Mouse } & \text { K. Nave, MPI of Exp. Med., Göttingen, Germany } \\ \text { Rab35 } & \text { Rabbit } & \text { A. Echard, Institut Curie, Paris, France }\end{array}$

Secondary antibodies were purchased from Dianova, Germany. Colloidal gold conjugates to protein A were obtained from the Cell Microscopy Center, Department of Cell Biology, Netherlands.

\subsubsection{Plasmids}

\subsubsection{Previously published plasmids}

The following expression plasmids were used: EGFR-EGFP (D. Arndt-Jovin, MPI for Bioph. Chem., Göttingen), EGFR (Uenal Coskun, MPI-CBG, Dresden), EGFP-CD63 (D. Cutler, University College London, UK), GFP-Vps4(KQ) and GFP-Vps4(EQ) (P. Woodman, University of Manchester, UK), VSVG-GFP and VSVG-SP(spacer)-GFP (P. Keller, MPI-CBG, Dresden), myc-Hrs (H. Stenmark, Institute of Cancer Research, Norway), MLV Gag- YFP (Addgene plasmid 1813; W. Mothes, Yale University School of Medicine, USA). EGFP-Rab-GAPs and their catalytically inactive mutants (Haas et al., 2005); EGFP-Rab GTPases and their mutants (Yoshimura et al., 2007); GFP-Rab5, GFP-Rab5 ${ }^{\text {Q79L }}$, GFP- 
Rab7 and GFP-Rab ${ }^{7 T 22 N}$ (M. Zerial, MPICBG, Dresden); pEYFP-C1 (BD Bioscience); PLP-myc and PLP-EGFP (Trajkovic et al., 2006).

\subsubsection{Self contructed plasmids}

The pcDNA3-HA-Hrs vector was generated by insertion of the HA tag-containing oligonucleotides into the EcoRI and HindIII restriction sites of the pcDNA3-myc-Hrs plasmid.

To produce DsRed2-Rab35 and its constitutively active and inactive mutations, Rab35 sequences were amplified from pEGFP-Rab35 plasmids with PCR (Primers: Rab35Fw and Rab35Rv), restriction digested and inserted into the pDsRed2-C1 plasmid (Invitrogen) between the Hind III and Bam HI restriction sites.

\subsubsection{Oligonucleotides and siRNA reagents}

\subsubsection{DNA primers}

Primer Sequence

Rab35Fw 5'-TTAAGCTTCGATGGCCCGGGACTACGACC-3'

Rab35Rv 5'-TTGGATCCTTAGCAGCAGCGTTTCTTTCGTTTACTG-3'

\subsubsection{2 $\quad$ siRNA}

\subsubsection{Commercial Kits}

$\begin{array}{ll}\text { Kit } & \text { Producer } \\ \text { Basic Neuron Neucleofector } & \text { Amaxa/Lonza } \\ \text { NucleoBond Xtra Midi } & \text { Machery-Nagel } \\ \text { NucleoSpin Plasmid } & \text { Machery-Nagel } \\ \text { QIAprep Spin Miniprep } & \text { Quiagen }\end{array}$




\subsubsection{Buffers, soultions, sera and media}

\subsubsection{Commercial media}

\section{Media}

B27 supplement

DMEM for Primary cell culture

DMEM for cell line culture

Fetal Calf Serum (FCS)

GlutaMAX $^{\mathrm{TM}}{ }_{-}$I supplement

Horse Serum

Insulin-Transferrin-Selenium - A supplement 100×

LB Medium

LB-Agar plate

PBS

Penicillin/Streptomycin (Pen/Strep) 100×

RPMI 1640

\section{Producer}

Gibco/Invitrogen

Gibco/Invitrogen

PAA

PAA

Gibco/Invitrogen

PAA

Gibco/Invitrogen

AppliChem

AppliChem

PAA

Gibco/Invitrogen

Gibco/Invitrogen

\subsubsection{PBS (phophate buffered saline)}

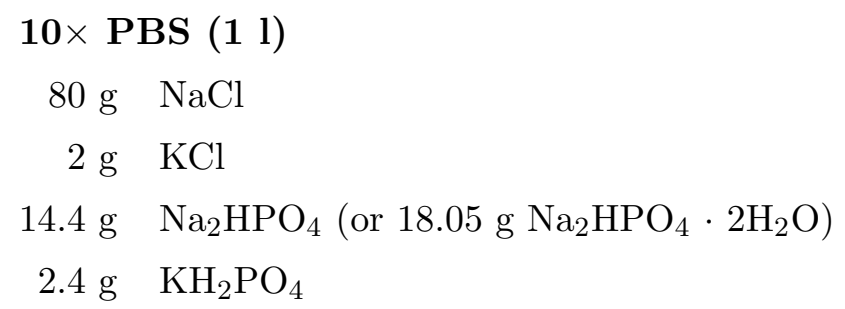

To obtain PBS, $10 \times$ PBS is diluted 10 times and its $\mathrm{pH}$ value is checked and adjusted to 7.2-7.4 if necessary. 


\subsubsection{SATO medium}

\section{Serum free SATO medium}

$\begin{aligned} 110 \mu \mathrm{g} / \mathrm{ml} & \text { pyruvate } \\ 5 \mu \mathrm{g} / \mathrm{ml} & \text { transferrin } \\ 10 \mu \mathrm{g} / \mathrm{ml} & \text { insulin } \\ 100 \mu \mathrm{M} & \text { putrescine } \\ 200 \mathrm{nM} & \text { progesterone } \\ 500 \mathrm{pM} & \text { tri-iodo-thyronine } \\ 220 \mathrm{nM} & \text { Na-selenite } \\ 520 \mathrm{nM} & \text { L-thyroxine } \\ 1 \times & \text { Pen/Strep } \\ 1 \times & \text { GlutaMAX }\end{aligned}$

in DMEM with high glucose and without glutamine

To obtain SATO medium, $5 \%$ horse serum is added to serum free SATO medium. The medium is then filter steriled.

\section{Super SATO medium}

$2 \%$ B27-supplement

$1 \%$ Horse serum

$110 \mu \mathrm{g} / \mathrm{ml}$ pyruvate

$1 \mu \mathrm{g} / \mathrm{ml}$ transferrin

$10 \mu \mathrm{g} / \mathrm{ml}$ insulin

$500 \mathrm{pM}$ tri-iodo-thyronine

$520 \mathrm{nM} \quad$ L-thyroxine

$1 \times \quad$ Pen/Strep

$1 \times$ GlutaMAX $^{\mathrm{TM}}$

in DMEM (Gibco/Invitrogen) with high glucose and without glutamine 
Stock solutions for SATO medium:

$\begin{array}{lll}\text { Component } & \text { Concentration } & \text { Storage } \\ \text { transferrin } & 5 \mathrm{mg} / \mathrm{ml} \text { in DMEM } & -20{ }^{\circ} \mathrm{C} \\ \text { insulin } & 1 \mathrm{mg} / \mathrm{ml} \text { in } 0.01 \mathrm{M} \mathrm{HCl} \text { in DMEM } & -20{ }^{\circ} \mathrm{C} \\ \text { putrescine } & 10 \mathrm{mM} \text { in DMEM } & -20{ }^{\circ} \mathrm{C} \\ \text { progesteron } & 2 \mathrm{mM} \text { in EtOH } & -20{ }^{\circ} \mathrm{C} \\ \text { pyruvate } & 1.1 \mathrm{~g} / \mathrm{ml} \text { in } \mathrm{H}_{2} \mathrm{O} & 4{ }^{\circ} \mathrm{C} \\ \text { tri-iodo-thyronine } & 500 \mathrm{nM} \text { in EtOH } & -20{ }^{\circ} \mathrm{C} \\ \text { Na-selenite } & 300 \mu \mathrm{M} \text { in } \mathrm{H}_{2} \mathrm{O} & 4{ }^{\circ} \mathrm{C} \\ \text { L-thyroxine } & 4 \mathrm{mM} \text { in } 0.13 \mathrm{~N} \mathrm{NaOH} \text { in } 70 \% \mathrm{EtOH} & 4{ }^{\circ} \mathrm{C}\end{array}$

Insulin-Transferrin-Selenium-A supplement can be used to substitute pyruvate, insulin, transferrin and Na-selenite

\subsubsection{Mowiol solution for immunocytochemistry}

To prepare the Mowiol solution, $2.4 \mathrm{~g}$ Mowiol was added to $6 \mathrm{~g}$ glycerol and $6 \mathrm{ml} \mathrm{H}_{2} \mathrm{O}$ was added to the mixture with agitation. After incubation at RT for $2 \mathrm{hr}, 12 \mathrm{ml} 0.2 \mathrm{M}$ Tris- $\mathrm{HCl}(\mathrm{pH} 8.5)$ was added and the mixture was incubated at $50-60{ }^{\circ} \mathrm{C}$ for $10 \mathrm{~min}$. The solution was then cleaned with centrifugation at $5000 \mathrm{~g}$ for $15 \mathrm{~min}$ and aliquoted. For longterm storage, the solution was kept at $-20^{\circ} \mathrm{C}$.

\subsubsection{Softwares}

\section{Software Website}

ApE http://www.biology.utah.edu/jorgensen/wayned/ape/

BibTeX http://www.bibtex.org/

ImageJ http://rsbweb.nih.gov/ij/

Inkscape http://www.inkscape.org/

JabRef http://jabref.sourceforge.net/

LaTeX http://www.latex-project.org/

R http://www.r-project.org/ 


\subsection{Methods}

\subsubsection{Cell culture, transfection, and RNA interference}

\subsubsection{Primary oligodendrocyte culture}

Oligodendroglial primary cell cultures from postnatal day 1 mouse were prepared as described previously (Fitzner et al., 2006). After shaking, cells were plated in Super SATO medium (Section 2.1.7.3) on $100 \mu \mathrm{g} / \mathrm{ml}$ poly-L-lysine (PLL) coated dishes at a density of $\sim 30,000$ cells $/ \mathrm{cm}^{2}$ (800 000 to 1000000 cells per petri dish with a diameter of $6 \mathrm{~cm}^{2}$ ). For collection of exosomes, the culture medium was changed to $1 \%$ SATO medium and the cells were cultured for $72 \mathrm{hr}$. The medium was further proceeded with the standard exosome purification method (Section 2.2.4).

\subsubsection{Growth and maintenance of cell lines}

The general cell culture incubation conditions used are $37{ }^{\circ} \mathrm{C}$ in $7.5 \% \mathrm{CO}_{2}$. The specific procedures for different cell lines are described below:

Oli-neu. The cells were plated on PLL pre-coated plastic petri dishes or glass coverslips and cultured in SATO medium with $5 \%$ serum (see Section 2.1.7.3). The surfaces were coated with $33 \mu \mathrm{g} / \mathrm{ml}$ PLL for 10 min (plastic) or $1 \mathrm{hr}$ (glass), washed with $\mathrm{H}_{2} \mathrm{O}$ and dried. The cells were grown to $\sim 80 \%$ confluence and washed off with fresh SATO medium. To keep the cell line in culture, the cells were usually passaged 1:5-1:6 every 2-3 days. Only the cells with passage number less than 35 were utilized for experiments.

PC3. The suspension culture of PC3 cells were kept in RPMI 1640 medium containing $10 \%$ FCS and $1 \times$ Pen/Strep. The cells were passaged 1:20 every 3-4 days.

\subsubsection{Freezing and thawing of cells}

For long-term storage of cell line, cells were grown to maximum confluence on a $10 \mathrm{~cm}$ petri dish or $75 \mathrm{~cm}^{2}$ cell culture flask and washed off or trypsinized as described in Section 2.2.1.2 according to the cell type. The cells were pellet with centrifugation at $900 \mathrm{~g}$ for $5 \mathrm{~min}$ and resuspended with $0.5 \mathrm{ml}$ DMEM and $0.5 \mathrm{ml} 2 \times$ freezing medium $(40 \%$ FCS, 20 \% DMSO in DMEM) was added subsequently to the cell suspension. The suspen- 
sion was mixed gently and transferred to a freezing vial. The cells were then frozen in a $-80{ }^{\circ} \mathrm{C}$ freezer within a freezing rack or a polystyrene block at temperature dropping rate of $-1{ }^{\circ} \mathrm{C} / \mathrm{min}$. After $\sim 16 \mathrm{hr}$ stored in the $-80{ }^{\circ} \mathrm{C}$ freezer, the cells were moved to a liquid nitrogen tank for long-term storage.

To resuscitation frozen cells, the cell ampoule was incubated in a $37{ }^{\circ} \mathrm{C}$ water bath immediately after taken form liquid nitrogen. The cell suspension was added to $9 \mathrm{ml}$ of fresh medium and then pelleted with centrifugation at $900 \mathrm{~g}$ for $5 \mathrm{~min}$. The cells were resuspended with fresh medium and plated or seeded to a $10 \mathrm{~cm}$ petri dish or $75 \mathrm{~cm}^{2}$ cell culture flask with previous coating, if necessary. After grown to maximum confluence, the cells were passaged normally as described in Section 2.2.1.2.

\subsubsection{Transfection}

Either FuGENE 6 or TransIT transfection reagent was used to deliver mammalian expression vector into the cells. The cells were cultured to $60-75 \%$ confluence on the day of transfection. Transfection reagent and plasmid DNA was added subsequently to OptiMem according to the culture vessel sizes (the amounts are described below) and the mixture was incubated at RT for 15-30 min. The mixture was added dropwise to the cells, the dish was shaken gently and the cells were incubated further in cultural condition for indicated period.

\begin{tabular}{|c|c|c|c|c|}
\hline Culture vessel & 12-well plate & 6-well plate & $3 \mathrm{~cm}$ dish & $10 \mathrm{~cm}$ dish \\
\hline Opti-MEM & $100 \mu \mathrm{l}$ & $150 \mu \mathrm{l}$ & $150 \mu \mathrm{l}$ & $600 \mu \mathrm{l}$ \\
\hline Transfection Reagent & $3 \mu \mathrm{l}$ & $4.5 \mu \mathrm{l}$ & $4.5 \mu \mathrm{l}$ & $18 \mu \mathrm{l}$ \\
\hline Plasmid DNA & $3 \mu \mathrm{g}$ & $4.5 \mu \mathrm{g}$ & $4.5 \mu \mathrm{g}$ & $18 \mu \mathrm{g}$ \\
\hline
\end{tabular}

\subsubsection{RNA interference}

To knockdown gene expression, siRNAs were delivered into Oli-neu cells by electroporation with Basic Neuron Neucleofector Kit in a two-step fashion. $2 \times 10^{6}$ cells were pelleted with centrifugation at $900 \mathrm{~g}$ for 5 min and resuspended with $100 \mu \mathrm{l}$ Neucleofector solution from the kit. $160 \mathrm{pmol}$ of siRNA was added to the suspension and delivered into the cell with electroporation program "O-05." The cells were then transferred to a pre-coated $10 \mathrm{~cm}$ dish with pre-warmed fresh SATO medium. After grown for 3 days under normal cell 
culture conditions, the cells were again collected and electroporated as described above. The cells were resuspended in fresh medium and plated onto culture vessels at a density of 150000 cells/well of 12 -well plate or 600000 cells per 3 -cm dish or well of 6 -well plate, with or without glass coverslips. The cells were transfeted with expressing plasmid in 2 days if desired and after 3 days incubation after the second electroporation, the cells were collected and subjected to further analysis.

\subsubsection{Molecular biology}

\subsubsection{Plasmid isolation from $E$. Coli}

The plasmid DNA from E. Coli was isolated and purified with NucleoBond Xtra Midi kit. The cells were inoculated in $100 \mathrm{ml}$ LB medium containing selecting antibiotics according to the plasmid (100 $\mu \mathrm{g} / \mathrm{ml}$ ampicillin or $50 \mu \mathrm{g} / \mathrm{ml}$ kanamycin) and incubated at $37{ }^{\circ} \mathrm{C}$ for $16 \mathrm{hr}$. The cells were collected with centrifugation at $\sim 3000 \mathrm{~g}$ for $20 \mathrm{~min}$ and the plasmid DNA was extracted and purified according to the manufacturer's protocol. The DNA was re-dissolved in $\mathrm{H}_{2} \mathrm{O}$ and the concentration was adjusted to $1 \mu \mathrm{g} / \mu \mathrm{l}$, if applicable.

For smaller amount (10-50 $\mu \mathrm{g})$, NucleoSpin Plasmid kit or QIAprep Spin Miniprep kit was used to purify DNA from $1.5 \mathrm{ml}$ bacterium culture.

\subsubsection{Restriction enzyme digestion}

The restriction enzyme digestions were performed with the buffer and enzyme system from New England Biolab or Fermentas. The general set-up of the reaction is described below:

\section{Restriction reaction mixture \\ 0.1-1 $\mu \mathrm{g}$ DNA \\ $2 \mu \mathrm{l} \quad 10 \times$ Buffer \\ 5-10 units each restriction enzyme \\ fill with $\mathrm{H}_{2} \mathrm{O}$ to a total volume of $20 \mu \mathrm{l}$}

where one unit is defined as the enzyme amount needed to digest $1 \mu \mathrm{g}$ lambda-DNA in one hour. The reaction mixture was then incubated for 1-4 hr at the reaction temperature indicated by the enzyme provider. 


\subsubsection{DNA ligation}

Ligation of two different DNA fragments were performed with buffer and enzyme system from Fermentas. To ligate a desired DNA fragment into a plasmid with sticky ends, the general set-up of the reaction is described below:

\section{Ligation mixture}

$\begin{array}{ll}1 \mu \mathrm{l} & 10 \times \text { buffer } \\ \text { 1-2 units } & \text { T4 DNA Ligase } \\ \text { 50-100 ng } & \text { Restriction enzyme cut vector DNA } \\ \text { 1:1-3:1 to vector }{ }^{1} & \text { Restriction enzyme cut insert } \\ \text { fill with } \mathrm{H}_{2} \mathrm{O} \text { to a total volume of } 10 \mu \mathrm{l}\end{array}$

where the unit represents Weiss unit (Weiss et al., 1968). The mixture was incubated at RT for 30-60 min and $5 \mu \mathrm{l}$ of the reaction mixture was used for further transformation.

\subsubsection{Polymerase chain reaction (PCR)}

The general reaction for polymerase chain reaction (PCR) was set up as described below:

\section{PCR mixture}

$\begin{array}{rl}x \mu \mathrm{l} & \text { template DNA solution } \\ 0.96 \mu \mathrm{l} & 25 \mathrm{mM} \mathrm{MgCl}_{2} \\ 1 \mu \mathrm{l} & 2.5 \mathrm{mM} \mathrm{dNTP} \\ 0.5 \mu \mathrm{l} & 10 \mathrm{mM} \text { Forward Primer } \\ 0.5 \mu \mathrm{l} & 10 \mathrm{mM} \text { Reverse Primer } \\ 0.2 \mu \mathrm{l} & \text { GoTaq DNA polymerase } \\ 4 \mu \mathrm{l} & 5 \times \text { PCR buffer } \\ \text { fill with } \mathrm{H}_{2} \mathrm{O} \text { to a total volume of } 20 \mu \mathrm{l}\end{array}$

The reaction mixture was loaded into a thin wall $200 \mu \mathrm{l}$ tube and applied to a thermocycler machine. The general program for the thermocycler machine was described below:

\footnotetext{
${ }^{1}$ molar ratio
} 


\section{Denaturing step}

$95^{\circ} \mathrm{C} \quad 3 \mathrm{~min}$

\section{Amplification cycles (25-32 cycles)}

$\begin{array}{ll}95{ }^{\circ} \mathrm{C} & 30 \mathrm{sec} \\ T_{\text {annealing }} & 30 \mathrm{sec} \\ 72{ }^{\circ} \mathrm{C} & t_{\text {amplification }}\end{array}$

\section{Completion step}

$72{ }^{\circ} \mathrm{C} \quad 5 \mathrm{~min}$

$4{ }^{\circ} \mathrm{C} \quad \infty$

where the $T_{\text {annealing }}$ stands for average annealing temperature of a specific primer pairs which was calculated with ApE program and $t_{\text {amplification }}$ represents the time duration for amplification of the sequence to be amplified, which can be calculated with know sequence length and the amplification rate of Taq polymerase estimated as $1 \mathrm{~kb} / \mathrm{min}$.

\subsubsection{Determine of DNA concentration}

To determine double-stranded DNA concentration, the DNA sample was diluted and the absorbance was read at $260 \mathrm{~nm}$. The concentration was calculated with the following formula:

$$
\text { Concentration of DNA }(\mu \mathrm{g} / \mathrm{ml})=A_{260} \times \text { dilution factor } \times 50 \mu \mathrm{g} / \mathrm{ml}
$$

\subsubsection{Transformation of $E$. Coli cells}

To transform chemical competent E. coli cells (strain: DH5 $\alpha$ ) with an plasmid, 40-50 $\mu$ l of competent cells were thawed on ice and 10-100 ng of the desired plasmid DNA was added into the cell suspension. After incubated further on ice for $2 \mathrm{~min}$, the cells were incubated in $500 \mu \mathrm{l} \mathrm{LB}$ medium at $37{ }^{\circ} \mathrm{C}$ with agitation for $1 \mathrm{hr}$. $100-200 \mu \mathrm{l}$ of the cell suspension was then plated onto agarose selection plates (containing $100 \mu \mathrm{g} / \mathrm{ml}$ ampicillin or $50 \mu \mathrm{g} / \mathrm{ml}$ kanamycin) and incubated at $37^{\circ} \mathrm{C}$ for $\sim 16 \mathrm{hr}$. 


\subsubsection{DNA sequencing and oligonucleotide synthesis}

DNA sequencing and oligonucleotide synthesis were performed by the DNA core facility of Max Planck Institute of Experimental Medicine, Göttingn, Germany.

\subsubsection{Protein biochemistry}

\subsubsection{SDS-PAGE}

To separate protein under denaturing condition with sodium dodecyl sulfate ployacrylamide gel electrophoresis (SDS-PAGE), a two-layer gel was set up with Bio-Rad MiniPROTEAN 3 casting system. The components of upper resolving gel and lower stacking gel are described below:
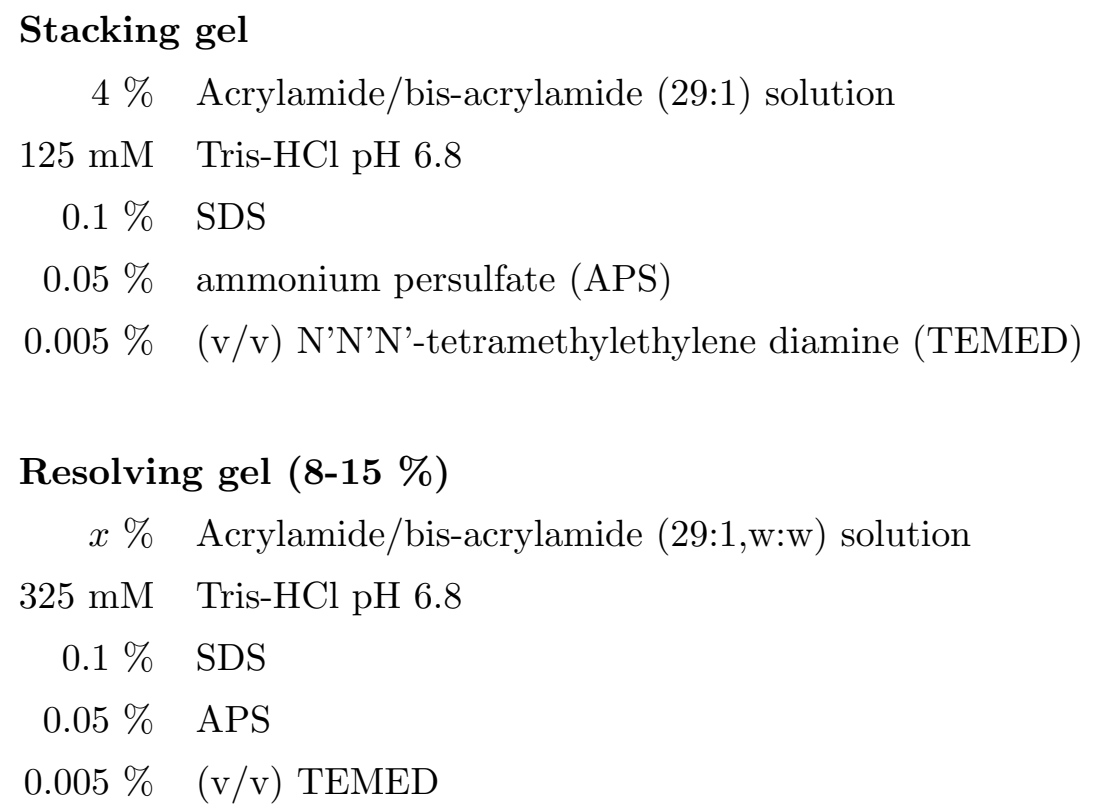

To cast the gel, the resolving gel was poured to $2 / 3$ height of the system, overlayed with $\mathrm{H}_{2} \mathrm{O}$ to ensure a horizontal edge, and incubated for $30 \mathrm{~min}$ at RT to complete the polymerizing reaction. After polymerization, $\mathrm{H}_{2} \mathrm{O}$ was removed and the stacking gel was poured and a comb was inserted to form the wells on the gel holding the protein samples.

The protein samples were dissolved in protein sample buffer (see below) and boiled at $95{ }^{\circ} \mathrm{C}$ for $5 \mathrm{~min}$ (or $70{ }^{\circ} \mathrm{C}, 10 \mathrm{~min}$ for PLP-EGFP; $55{ }^{\circ} \mathrm{C}, 10 \mathrm{~min}$ for PLP-myc to avoid 
dimerization) to denature the proteins.

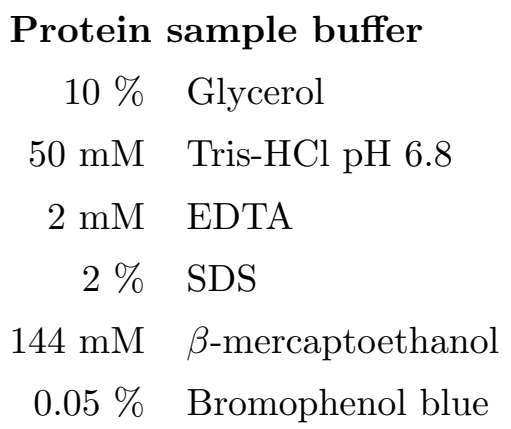

The gel was set up onto a Bio-Rad mini gel electrophoresis system filled with PAGE buffer (see below). The sample was loaded onto the gel and the electrophoresis was performed with $70 \mathrm{~V}$ for stacking and $100 \mathrm{~V}$ for resolving.

\section{PAGE buffer}

$0.25 \mathrm{M}$ Tris base

$1.92 \mathrm{M}$ Glycine

$1 \%$ SDS

\subsubsection{Western Blot}

Western blot was performed to detect target protein after electrophoresis with antibodies. After seperation with SDS-PAGE, the proteins were transfered to a nitrocellular membrane using the Bio-Rad Mini-Protein System. The gel sandwich in blotting cassette was set up as indicated in manual. The cassette was placed in the tank of the module filled with transfer buffer (0.25 M Tris base, $1.92 \mathrm{M}$ glycine, $20 \%$ methanol) and applied with $100 \mathrm{~V}$ for $1 \mathrm{hr}$ to transfer the proteins onto the membrane.

After transfer, the membrane was washed in PBST (0.1\% Tween 20 in PBS) for 10 min, immersed in blocking solution (4\% skim milk powder in PBS) for $30 \mathrm{~min}$ at RT and incubated with primary antibody in PBST at RT for $1 \mathrm{hr}$ or at $4{ }^{\circ} \mathrm{C}$ for $\sim 16 \mathrm{hr}$. The membrane was then washed $3 \times 5$ min at RT with PBST, incubated with horse radish peroxidase (HRP) conjugated secondary antibody in blocking solution for 30 min at RT, and washed $3 \times 5$ min at RT with PBST. 
The targeted protein was detected with enhanced chemiluminescence (Pierce/Thermo Scientific) After the wash steps, the membrane was covered with the substrate and incubated for $1 \mathrm{~min}$ at RT. The fluorescence was detect with X-ray films, which were scanned with a conventional scanner and the signal densities of the bands were analyzed with ImageJ.

\subsubsection{Exosome purification}

Exosomes were collected with step centrifugation. The medium was changed to serum free medium and the cells were further incubated in general culture conditions for $4 \mathrm{hr}$ or indicated collection period. For clearance of other larger cellular membrane structures, the medium was then collected and subjected to centrifugation steps: $3000 \mathrm{~g} \times 10 \mathrm{~min}$, $2 \times 4000 \mathrm{~g} \times 10 \mathrm{~min}$ and $10000 \mathrm{~g} \times 30 \mathrm{~min}$. After the clearance steps, the medium was further centrifuged at $100000 \mathrm{~g}$ for $1 \mathrm{hr}$ and the pellet was re-dissolved with protein sample buffer (Section 2.2.3.1).

To compare the amount of a specific protein released with exosomes, the cells, from which the exosomes were collected, were lysed with cell lysis buffer (see below), scraped off from the petri dish with cell scraper. For PC3 cells, the cells were collected with centrifugation at $900 \mathrm{~g}$ for $5 \mathrm{~min}$ and the cell lysis buffer was added to the pellet. After incubated on ice for $10 \mathrm{~min}$, the lysate was then centrifuged at $10000 \mathrm{~g}$ for $10 \mathrm{~min}$ and the supernatant was collected. Same volume of $2 \times$ protein sample buffer, whose components have twice concentration as the protein sample buffer, was added to the lysate. If not indicated, the total amount of exosome sample and $5 \%$ of cell lysate sample were subjected to SDSPAGE followed with Western Blot based analysis.

Sucrose step gradient was performed to separate the proteins of the exosome samples based on the densities of the membrane structure where they located. The pellet collected from the step centrifugation was resuspended with 2.5M sucrose in $20 \mathrm{mM}$ HEPES buffer (pH 7.4) and overlayed with a step gradient of sucrose $(2.25,2,1.75,1.5,1.25,1,0.75$, 0.5 and $0.25 \mathrm{M}$ in $20 \mathrm{mM}$ HEPES buffer, $\mathrm{pH} 7.4$ ). The gradient was then centrifuged at $200000 \mathrm{~g}$ for $3 \mathrm{hr}$ and fractions were collected from the top of the gradient and analyzed with SDS-PAGE/Western Blot. 


\subsubsection{Immunocytochemistry and microscopy}

\subsubsection{Immunocytochemistry}

To label the cells with different fluorescence markers, immunocytochemistry was performed at RT. The cells grown on glass coverslip were washed 2 times with PBS and fixed with $4 \%$ paraformadehyde in PBS for 15 min. After washed with PBS, they are further permeabilized with 0.1 \% (v/v) Triton X-100 for 1 min, washed 3 times with PBS, and incubated in blocking solution (see below) for $30 \mathrm{~min}$. The cells were then incubated in primary antibody solution (antibodies in $10 \%$ blocking solution, diluted with PBS) for $1 \mathrm{hr}$, washed 3 times and incubated in secondary antibody solution for 30-60 min. After 3 times washed with PBS, the glass coverslip was mount onto a glass slide with a drop of Mowiol solution (Section 2.1.7.4) and dried overnight in the dark.

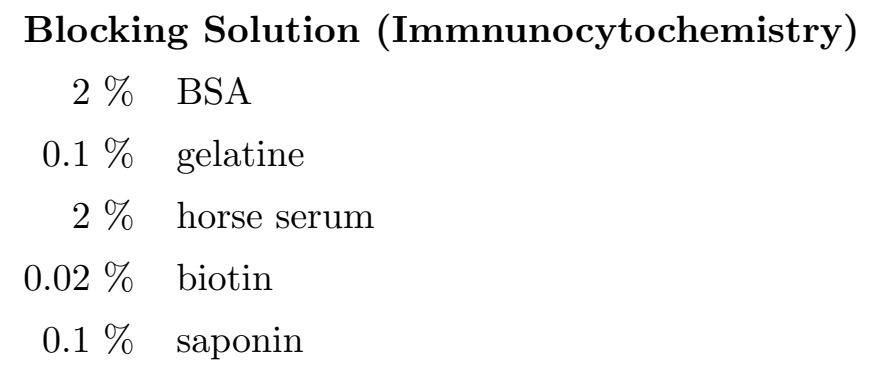

\subsubsection{Confocal Microscopy}

Confocal microcopic images of fixed cell samples were aquired with Carl Zeiss LSM 510 microscope with a $63 \times$ oil plan-Apochromat objective, or with Leica DMIRE2 microscope with a $63 \times$ oil-immersion objective and a Leica TCS SP2 AOBS confocal laser scanning setup.

For live cell imaging, the cells were washed and incubated with imaging medium (see below) and the images were obtained at $37{ }^{\circ} \mathrm{C}$ with the Leica setup as described above. The temperature was controlled with an air flow chamber. The cells were imaged in the condition up to $45 \mathrm{~min}$. 


\section{Imaging medium

$\begin{array}{rl}160 \mathrm{mM} & \mathrm{NaCl} \\ 4.5 \mathrm{mM} & \mathrm{KCl} \\ 2 \mathrm{mM} & \mathrm{CaCl} 2 \\ 1 \mathrm{mM} & \mathrm{MgCl} 2 \\ 10 \mathrm{mM} & \text { glucose } \\ 10 \mathrm{mM} & \text { HEPES, pH } 7.4\end{array}$

\subsubsection{TIRF Microscopy}

To observe the structures close to the plasma membrane, total internal reflection fluorescence (TIRF) microscopy was performed with a prism based set-up. The images were acquired with a Photometrics Cascade 512B camera on a Carl Zeiss Axiovert 135 microscope with a $63 \times$ water immersed objective (Leica). The excitation light was generated with an Ar laser (488 nm) and the fluorescence emission signals were filtered with a HQ $515 / 30$ band-pass filter.

The cells were washed and incubated with imaging medium (Section 2.2.5.2) and the images were obtained at RT. For comparison, wide-filed fluorescence images were obtained with the same capturing settings as described above, together with an 450-490 band-passed excitation light generated with a monochromator.

\subsubsection{Electron microscopy}

For electron microscopy analysis for exosomes, cells were transiently transfected with PLP, exosomes were prepared, fixed with $2 \%$ paraformaldehyde and were adsorbed to glowdischarged formvar-carbon-coated nickel grids. The samples were post-fixed for $2 \mathrm{~min}$ with $1 \%$ glutaraldehyde and immunolabelled with anti-3F4 antibody followed by $10 \mathrm{~nm}$ goldlabelled secondary antibody. As a control for unspecific binding untransfected cells were used. After embedding in methylcelluloseuranyl acetate, samples were imaged with a CM120 Philips electron microscope equipped with a TVIPS camera.

immunoelectron microscopic assay on CNS myelin was performed as described (Werner et al., 2007). Mice were fixed by transcardial perfusion with $4 \%$ formaldehyde and $0.2 \%$ 
glutaraldehyde in $0.1 \mathrm{M}$ phosphate buffer containing $0.5 \% \mathrm{NaCl}$. Dissected optic nerves were infiltrated in $2.3 \mathrm{M}$ sucrose in $0.1 \mathrm{M}$ phosphate buffer over night, mounted onto aluminum pins for ultramicrotomy and frozen in liquid nitrogen. Ultrathin cryosections were picked up according to (43) in a 1:1 mixture of $2 \%$ methylcellulose and $2.3 \mathrm{M}$ sucrose. For immuno-labeling, sections were incubated with antibodies specific for PLP, which was detected with protein A-gold (10 nm). Sections were analyzed with a LEO EM912 Omega (Zeiss, Oberkochen) and digital micrographs were obtained with an on-axis 2048x2048CCD camera (Proscan, Scheuring).

\subsubsection{Image Analysis}

\subsubsection{Colocalization analysis on endosomal membrane}

Image processing and analysis were performed using Meta Imaging Series 6.1 software (Universal Imaging Corp.). Quantification of colocalization was performed with the colocalization module of the software as described previously (Westphal et al., 2008). This function calculates the area of the region of overlap between two fluorescent probes (area overlap). The area of overlap for PLP with the respective marker proteins on individual endosome is reported as the percentage of total anti-PLP labelled area of an individual endosome. A fixed threshold was used to eliminate the low intensity background signal in both channels. At least three independent experiments were performed for each analysis and 20-30 cells imaged in each experiment. Transfected cells were randomly selected and images were recorded by zooming into the area containing the endosomes and by using below pixel-value saturation for the recordings in each channel. For the quantification of colocalization, only endosomes that contained both markers in similar intensities were used for the analysis.

\subsubsection{Quantification of signals within the endosomal lumen and on the lim- iting membrane}

To quantify the amount of PLP, EGFR or VSV-G within the lumen and the limiting membrane of the Rab5 ${ }^{\mathrm{Q} 79 \mathrm{~L}}$ enlarged endosomes, confocal sections of endosomes were taken using the GFP-Rab5 ${ }^{\mathrm{Q} 79 \mathrm{~L}}$ outline as a reference to obtain central images of the endosomes. For the quantification the GFP-Rab5 ${ }^{\mathrm{Q} 79 \mathrm{~L}}$ outline served as a mask to determine the local- 
ization of the limiting membrane. The fluorescence intensities of the respectively labeled proteins were determined in the lumen and the limiting membrane of the endosomes and quantified using Meta Imaging Series 6.1 software.

\subsubsection{Vesicle tracking}

To analyze vesicular movement, PLP-myc was Co-transfected with wild-type or mutant EGFP tagged Rab35, cells were stained with 50 nM LysoTracker Red DND-99 (Invitrogen) for $10 \mathrm{~min}$ 24-30 h after transfection, washed with imaging medium (Section 2.2.5.2), and the time-lapse images were acquired with a Leica confocal microscope at 1 frame every $2 \mathrm{sec}$ at $37^{\circ} \mathrm{C}$. The pinhole was set at $200 \mu \mathrm{m}$ and a pixel size of $0.12 \mu \mathrm{m} \times 0.12 \mu \mathrm{m}$ was used. The velocities of the vesicles were automatically analyzed using a previously described algorithm (Westphal et al., 2008). The images were smoothened by convolution with a Gaussian function of 2 pixels full width at half maximum and vesicles were found as the local maxima, which exceed a threshold of $20 \%$ relative to the brightest object. Vesicles are tracked by minimizing the sum of the squared distances moved of all particles. The maximally allowed speed of a particle is 8 pixels/frame.

\subsubsection{Correlation analysis}

Pearson's correlation coefficient $R_{x y}$ between two channels (images) $x$ and $y$ can be calculated with the following equation:

$$
R_{x y}=\sum_{i=1}^{n} \frac{\left(x_{i}-\bar{x}\right)\left(y_{i}-\bar{y}\right)}{\sqrt{\sum_{i=1}^{n}\left(x_{i}-\bar{x}\right)^{2} \sum_{i=1}^{n}\left(y_{i}-\bar{y}\right)^{2}}}
$$

where $x_{i}$ and $y_{i}$ are the values of pixel $i$ in channels $x$ and $y$.

For PLP/Lamp-1 colocalization assay, an 8-bit confocal image with a resolution of 0.071 $\mu \mathrm{m} /$ pixel of a $7 \mu \mathrm{m} \times 7 \mu \mathrm{m}$ intracellular region was obtained; signals of PLP-myc and Lamp-1 channels of the image were subjected to a calculation using a self-written ImageJ plugin for Pearson's correlation coefficient (Appendix B.1). To calculate vesicle size and number, confocal images with a pixel size as above of a $73 \mu \mathrm{m} \times 73 \mu \mathrm{m}$ field were taken and analyzed with ImageJ.

For the correlation change in image series acquired with the TIRF microscope, an image region of $15.2 \mu \mathrm{m} \times 15.2 \mu \mathrm{m}$ containing cells expressing DsRed2-Rab35, identified in the 
wide field fluorescence channel, was cropped and Pearson's correlation coefficient was calculated between pairs of images separated by time (first frame and the following frames within the video sequence, Appendix B.2).

\subsubsection{EGF degradation}

Cells transfected with EGFR-EGFP were incubated with $50 \mathrm{ng} / \mathrm{ml}$ Alexa555-labelled EGF (Invitrogen, Eugene, OR) for $15 \mathrm{~min}$, washed, and incubated for $4 \mathrm{~h}$ in conditioned culture medium to allow EGF degradation. For quantification, images of randomly selected transfected cells were recorded at fixed settings below pixel-value saturation and fluorescence intensities were quantified using Meta Imaging Series 6.1 software.

Alternatively, cells were labelled or $15 \mathrm{~min}$ at room temperature with $6 \mathrm{ng} / \mathrm{ml}$ of $\left[{ }^{125} \mathrm{I}\right] \mathrm{EGF}$ in SATO medium with $10 \mathrm{mM}$ Na-HEPES, $0.1 \%$ BSA, pH 7.4, washed and chased with the same medium at $37^{\circ} \mathrm{C}$. After 0, 15, 90 and $180 \mathrm{~min}$, TCA (10\%)-soluble radioactivity was measured in the extracellular medium with a $\gamma$-counter to determine the degradation of $\left[{ }^{125} \mathrm{I}\right] \mathrm{EGF}$ (Futter et al., 1996).

\subsubsection{Myelin preparation}

The myelin preparation was performed essentially as described (Larocca \& Norton, 2007). Briefly, the brains from 3-6 month old mice were homogenized in PBS containing protease inhibitors by sonication. The brain homogenate was put on top of two-step sucrose gradients $(0.32 \mathrm{M}$ and $0.85 \mathrm{M}$ sucrose in preparation buffer containing $5 \mathrm{mM}$ EDTA and $10 \mathrm{mM}$ HEPES, pH 7.4). After the gradients were centrifuged at $75000 \mathrm{~g}$ for $30 \mathrm{~min}$, the interfaces were collected, diluted at least 10 times with $\mathrm{H}_{2} \mathrm{O}$ and centrifuged at $75000 \mathrm{~g}$ for 20 min. The pellet was washed twice with $\mathrm{H}_{2} \mathrm{O}$ and recovered by 10 min centrifugation at $12000 \mathrm{~g}$ (crude myelin) and the protocol was repeated to obtain purified myelin. To separate the heavy and light myelin (Rios et al., 2000), the crude myelin pellet was re-suspend with preparation buffer, put on top of a three-step sucrose gradient (0.32 M, $0.62 \mathrm{M}$ and $0.8 \mathrm{M}$ sucrose in preparation buffer) and centrifuged at $75000 \mathrm{~g}$ for $30 \mathrm{~min}$. The light and heavy myelin fractions were collected at the 0.32/0.62 $\mathrm{M}$ and $0.62 / 0.8 \mathrm{M}$ sucrose interfaces, respectively. The fractions were washed with $\mathrm{H}_{2} \mathrm{O}$ and recovered by centrifugation for $20 \mathrm{~min}$ with $75000 \mathrm{~g}$ and twice $10 \mathrm{~min} \times 12000 \mathrm{~g}$ as described above. The protein 
concentrations of the brain homogenate and myelin preparations were determined by the Bradford assay (Bradford, 1976). 


\title{
Chapter 3
}

\section{Results}

\subsection{Ceramide triggers budding of vesicles into multivesicular bod- ies for the release as exosomes}

Most of the results in Section 3.1 have been published in:

\author{
Ceramide triggers budding of exosome vesicles into multivesicular endosomes \\ Katarina Trajkovic*, Chieh Hsu*, Salvatore Chiantia, Lawrence Rajendran, Dirk \\ Wenzel, Felix Wieland, Petra Schwille, Britta Brügger and Mikael Simons \\ Science 319(5867):1244-7 (2008) \\ *These authors contributed equally to this work
}

\subsubsection{Oli-neu cells secrete exosomes}

In this study, we examined the question of how exosomes are generated.

To address this issue, we studied the membrane trafficking of the proteolipid protein (PLP) in Oli-neu cells, an oligodendroglial cell line that contains a large number of MVBs (Trajkovic et al., 2006). PLP, the major protein of myelin of the central nervous system, is a four-membrane spanning, palmitoylated, cholesterol-binding protein of $26 \mathrm{kD}$ that is mainly found in MVBs of Oli-neu cells (Simons et al., 2000; Trajkovic et al., 2006). To analyze whether PLP is released in association with exosomes, the cell culture medium of transiently transfected Oli-neu cells and oligodendrocytes were subjected to sequential centrifugation steps with increasing centrifugal forces to final obtain a $100000 \mathrm{~g}$ pellet, which mainly contained small membrane vesicles with a size of approximately 50-100 nm (Figure 3.1 A, B), similar to previously described exosomes. Relatively large amounts of 
PLP were found in the $100000 \mathrm{~g}$ pellet (Figure $3.1 \mathrm{~B}$ ) and immunoelectron microscopy analysis revealed the presence of PLP on the vesicles (Figure 3.2). In contrast, PLP containing the cytotoxic, missense mutation $(\mathrm{A} 242 \mathrm{~V})$ that leads to misfolding of the protein and to retention in the endoplasmic reticulum (ER) was not detected in the $100000 \mathrm{~g}$ pellet (Figure $3.1 \mathrm{~B}$ ), excluding cell lysis as a major contributing factor. To further rule out the possibility of membrane release as a consequence of cell lysis, the $100000 \mathrm{~g}$ pellet was analyzed for the presence of calnexin, $\gamma$-adaptin, GM130 and EEA1, membrane markers of the ER, TGN, cis-Golgi-matrix and early endosomes, respectively. These proteins were not detected in the $100000 \mathrm{~g}$ pellet, which did contain the two exosomal marker proteins, Alix and flotillin (Figure 3.3 A). Furthermore, we exogenously expressed a variety of integral membrane proteins, the EGF receptor (EGFR), the vesicular stomatitis virus glycoprotein (VSV-G) (both a wild-type and a endocytosis-deficient mutant form) and the amyloid precursor proteins (APP), but did not detect any of these proteins in the $100000 \mathrm{~g}$ pellet, from which PLP was readily recovered, demonstrating the specificity of the sorting process (Figure $3.3 \mathrm{~B}$ ). To further substantiate the association of PLP with exosomes, we analyzed the $100000 \mathrm{~g}$ pellet in a continuous sucrose density gradient and found that the majority of PLP was enriched in the same fraction as the exosomal marker protein Alix (Figure $3.1 \mathrm{C}$ ). Similar results were obtained when PLP-EGFP-stably expressing Oli-neu cells were used for the analysis (Figure $3.1 \mathrm{C}$ ). Exosomes are thought to be of endosomal origin and to derive by fusion of MVBs with the cell surface. To explore whether PLP requires transport through the endosomal system to be released with exosomes we cotransfected PLP with the early-endosomal GTPase-deficient Rab5 (Rab5 ${ }^{\mathrm{Q} 79 \mathrm{~L}}$ ) to impair intra-endosomal trafficking (Stenmark et al., 1994). We found that a large fraction of PLP was entrapped in the enlarged early endosomes and that the release of PLP by exosomes was significantly reduced (Figure 3.4).

These findings demonstrate that PLP containing exosomes are derived from the endosomal system and raises the question of how exosomal cargo segregates from non-exosomal cargo in endosomes.

\subsubsection{Formation of endosomal subdomains}

To study the possible segregation of cargo within distinct endosomal microdomains, cells were transfected with Rab5 ${ }^{\mathrm{Q} 79 \mathrm{~L}}$ to enlarge early endosomes and to facilitate domain in- 

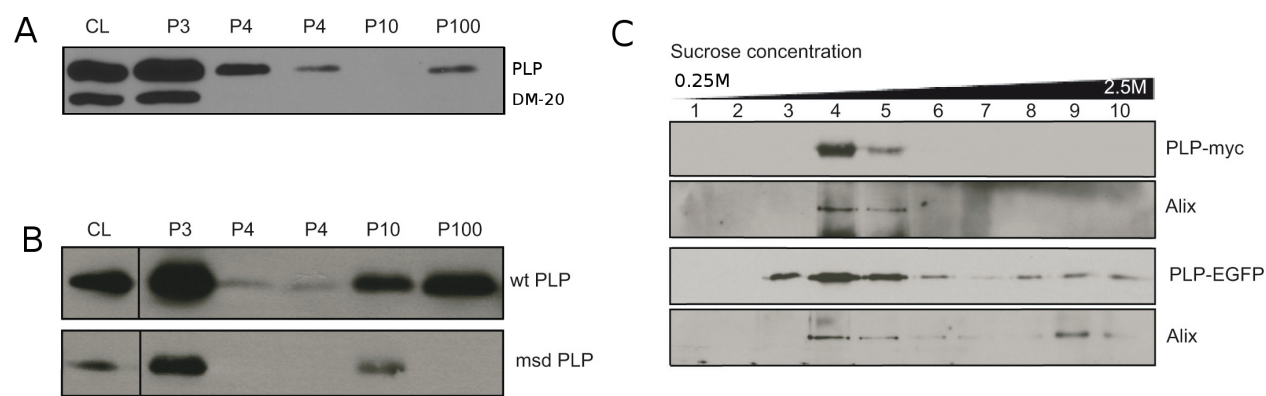

Figure 3.1: PLP was detected in exosomes biochemically. (A) After isolated from mixed brain culture, oligodendrocytes were incubated for $72 \mathrm{hr}$; (B) Oli-neu cells were transiently transfected with myc-tagged, wild-type (wtPLP) or mutant (msd-PLP) PLP; the medium was collected and submitted to sequential centrifugation steps as indicated. The resulting pellets of each centrifugation step were analyzed by Western blotting for PLP. (C) A 0.25 to $2.5 \mathrm{M}$ sucrose gradient was loaded on top of the $100,000 \mathrm{~g}$ pellet, and the resulting fractions were analyzed for PLP from transiently (PLP-myc) or stably transfected (PLP-EGFP) cells and for the exosomal protein Alix.
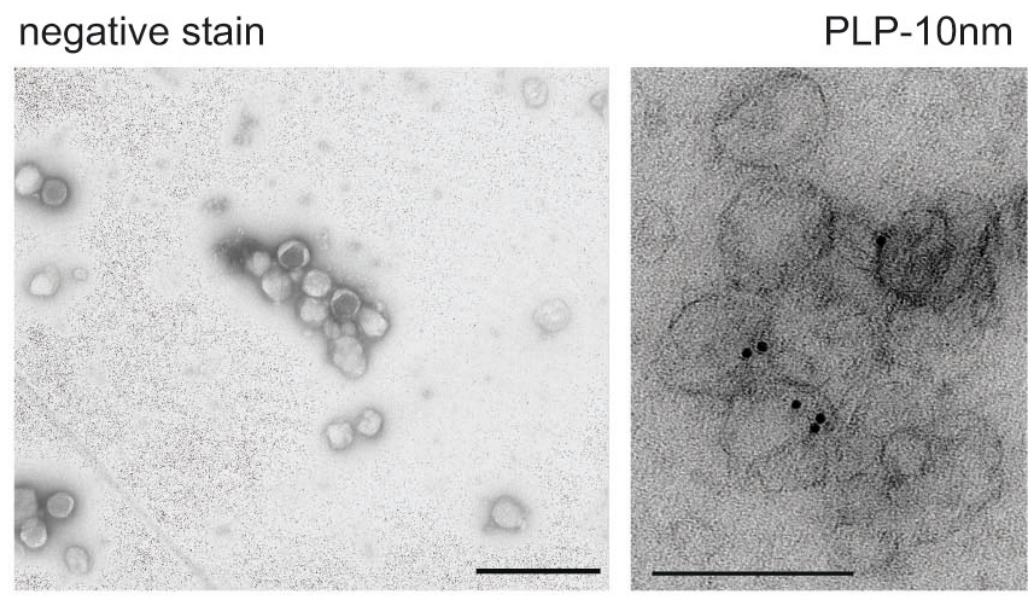

Figure 3.2: Ultrastructure of the exosomes secreted by Olin-neu cells. Cells were transiently transfected with PLP-myc; $100000 \mathrm{~g}$ pellets were prepared and negatively stained with $1 \%$ uranyl acetate and immunolabeled with antibodies against PLP (right). Scale bar, $200 \mathrm{~nm}$. 


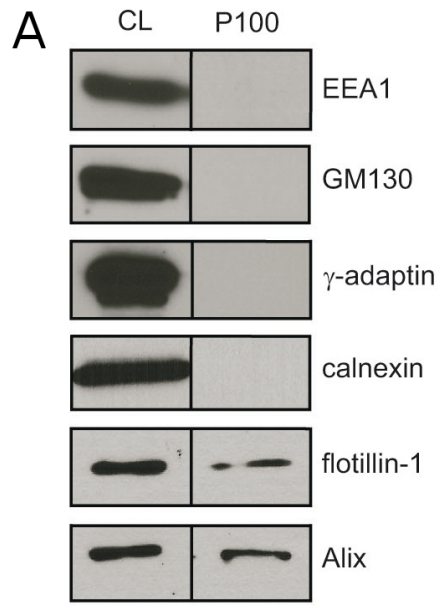

B

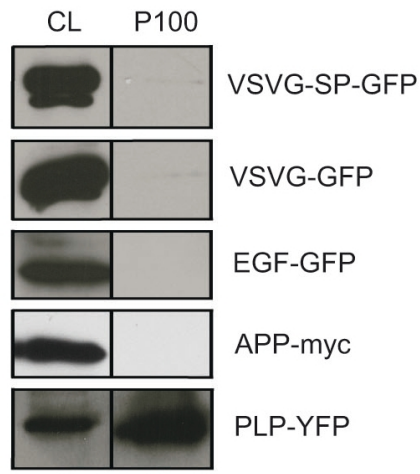

Figure 3.3: Endogenous and exogenous proteins in exosomes. Exosomes were isolated by sequential centrifugation steps as described in Material and Methods. (A) Cells were transiently transfected and cell lysates and $100000 \times \mathrm{g}$ pellets were analyzed for a variety of exogenously expressed integral membrane proteins. The EGF receptor (EGFR), the vesicular stomatitis virus glycoprotein (VSV-G) (both an apical (VSVG-GFP) and a basolateral (VSVG-SP-GFP) version) and the amyloid precursor protein (APP) were not detected in the $100000 \mathrm{~g}$ pellet, from which PLP was readily recovered. (B) The cell lysates (CL) and $100000 \mathrm{~g}$ pellets (P100) were analyzed by Western blotting for the presence of calnexin, $\gamma$-adaptin, GM130 and EEA1, membrane markers of the ER, TGN, cis-Golgi matrix and early endosomes, respectively. These proteins were not detected in the $100000 \mathrm{~g}$ pellet, which did contain the two exosomal proteins, Alix and flotillin.

A

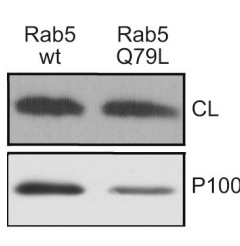

B

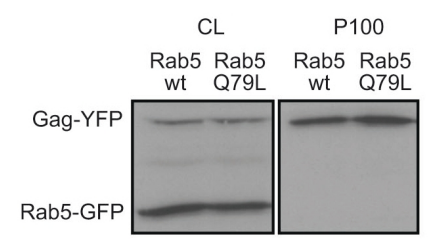

C

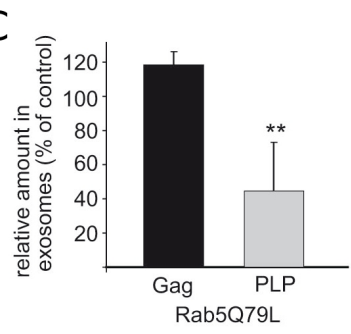

Figure 3.4: Rab5 ${ }^{\mathrm{Q79L}}$ impaired PLP release with exosomes. Cells were cotransfected with either GFP-Rab5 ${ }^{\text {Q79L }}$ or wild-type GFP-Rab5 (wt) and PLP-myc (A) or Gag-YFP (B); the amount of PLP and Gag in the cell lysates and 100,000g pellets was determined and quantified (C). Results are expressed as the mean \pm SD of five experiments $\left({ }^{* *} p<0.01\right.$; one-sample $t$ test against $100 \%$ ). 

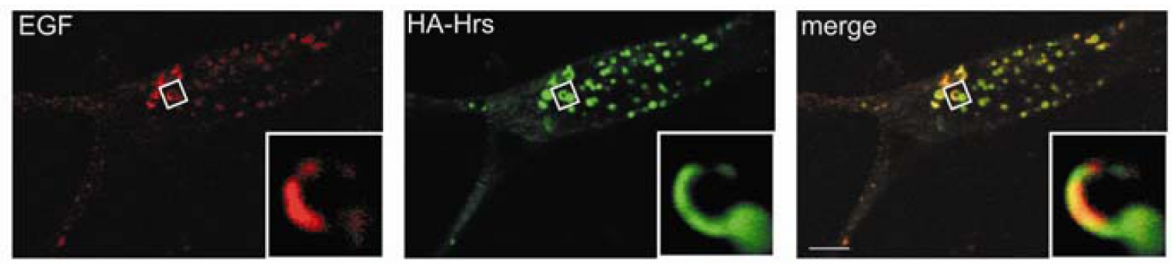

Figure 3.5: Colocalization of EGF and HA-Hrs on endosomes. Oli-neu cells were cotransfected with EGFR and HA-Hrs, cells were then incubated for 15 min with Rhodamine-labelled EGF and processed and analyzed for immunofluorescence microscopy. Insets show the endosomes. Scale bar, $10 \mu \mathrm{m}$.

spection by confocal immunofluorescence analysis (Raiborg et al., 2002). Previous studies have identified clathrin-coated microdomains on early endosomes that contain the ubiquitin-binding protein Hrs (hepatocyte-growth-factor regulated tyrosine kinase substrate), which sorts ubiquitanted proteins into these domains to mediate degradative protein sorting (Raiborg et al., 2006). The EGFR is a prototypic example of a receptor that requires Hrs-mediated sorting. To analyze whether PLP localizes to these microdomains, we analyzed the localization of PLP in Rab5 ${ }^{\text {Q79L }}$ enlarged endosomes. There was little colocalization of PLP with either Hrs or EGFR (visualized by a 15 min incubation with Rhodamine-conjugated EGF), whereas consistent with previous studies Hrs colocalized to some extent with EGFR (Figure 3.5). To characterize the nature of the PLP enriched domain, we analyzed the degree of colocalization with flotillin, a membrane scaffolding protein of non-caveolar lipid-raft microdomains. Confocal microscopy analysis revealed a strong colocalization of PLP with flotillin (Figure 3.6). Likewise, we also found that GPIGFP colocalized with PLP in the enlarged endosomes again suggesting that segregation of PLP from EGFR involves lipid raft-based microdomain (Figure 3.6).

\subsubsection{ESCRT-independent formation of exosomes}

Having shown that EGFR and PLP are found on distinct domains in endosomes, we next addressed the question whether they use distinct pathways of inward budding in endosomes. It is well established that sorting of EGFR into ILVs requires the sequential action of different components of the endosomal sorting complex required for transport (ESCRT) machinery (Hurley \& Emr, 2006; Williams \& Urbé, 2007). As an assay for inward budding, we determined the amount of EGF being transferred to the lumen of the enlarged endo- 

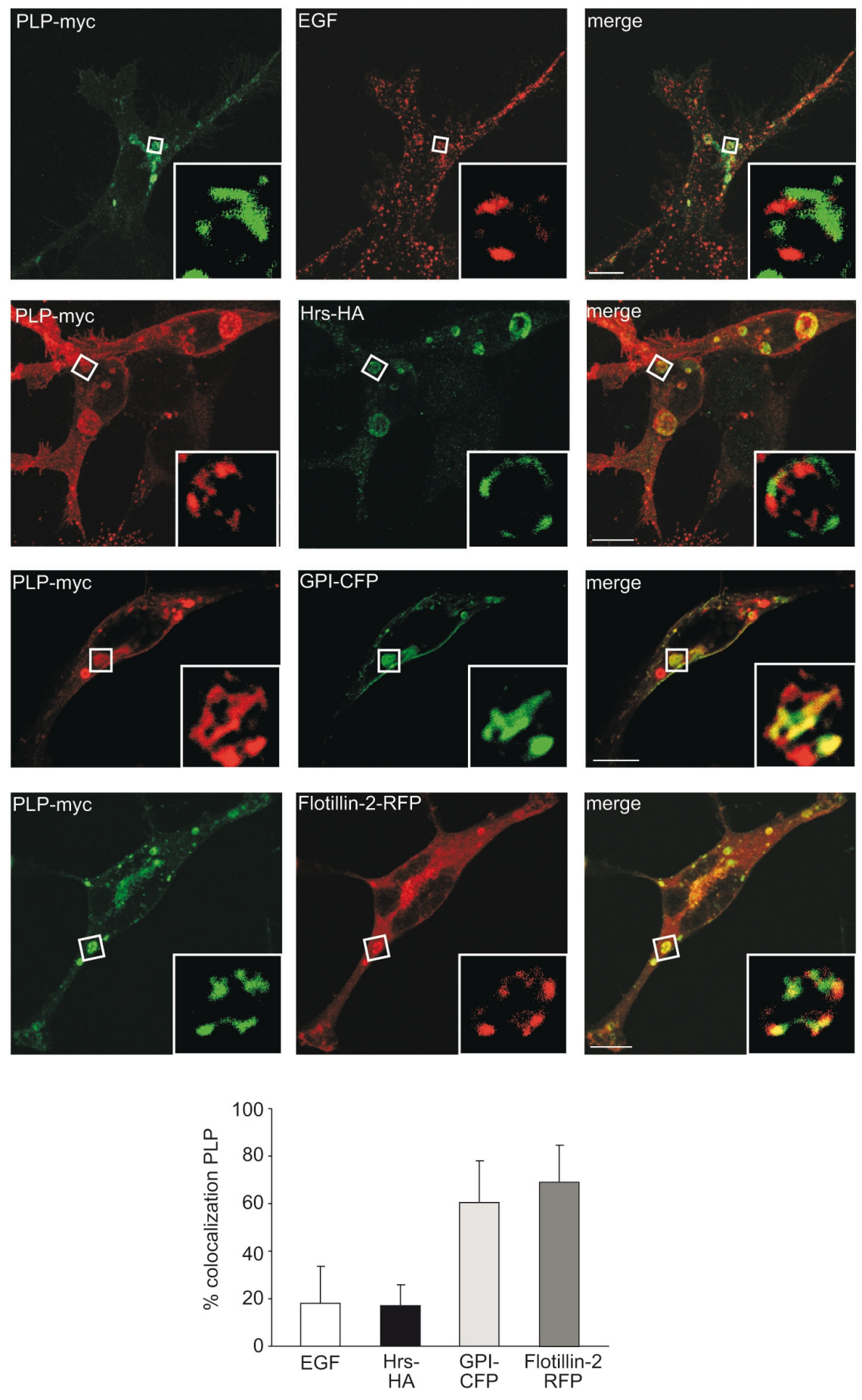

Figure 3.6: Endosomal subdomain structure. Oli-neu cells were cotransfected with Rab5 ${ }^{\mathrm{Q} 79 \mathrm{~L}}$ and PLP-myc, EGFR, hemagglutinin (HA) epitope-tagged Hrs (HA-Hrs), GPI-CFP, or flotillin-2 fused with red fluorescent protein (flotillin-2-RFP) as indicated. Cells transfected with EGFR were incubated for $15 \mathrm{~min}$ with rhodamine-labeled EGF. Cells were then processed and analyzed for immunofluorescence microscopy. The white box indicates the (inset) region reimaged in higher resolution and contrast to resolve the subdomain structure. Quantification of colocalization of the different proteins on endosomemembranes is shown ( $\mathrm{n}=\sim 25$ endosomes). Scale bar, $10 \mu \mathrm{m}$. 
somes after EGF stimulation. To interfere with the function of the ESCRT machinery, we used either RNAi or dominant-negative mutants against Hrs, Tsg101, Alix or Vps4, respectively. An efficient knock-down of protein was obtained by performing two rounds of siRNA delivery using nucleofection (Figure $3.7 \mathrm{~A}$ ). We found that depletion of either Hrs or Tsg101 significantly reduced the intraluminal transport of EGFR into enlarged endosomes, whereas Alix depletion only had a minor effect (Figure 3.7 B). The reduction of EGF in the endosomal lumen correlated with a defect in EGF degradation (Figure 3.8). These results are consistent with previous data validating our inward budding assay (Razi \& Futter, 2006; Bache et al., 2003; Lloyd et al., 2002; Babst et al., 2000; Bishop \& Woodman, 2000; Yoshimori et al., 2000). We also analyzed the effect of the Tsg101 knock-down on intra-endosomal transport of VSV-G to test the assay for ligand-independent transport and found that depletion of Tsg101 reduced the amount of VSV-G within the lumen of the endosomes (Figure 3.9).

To analyze the involvement of the ESCRT-machinery in transport of PLP, similar experiments were performed for PLP. We found that neither knock-down of Hrs, Tsg101 nor Alix had any influence on the inward budding of PLP (Figure 3.7). In addition, the functional inhibition of the ESCRT-machinery did not change the co-localization of PLP with Lamp-1 (Figure 3.10). Together, these results suggest an ESCRT-independent pathway for intra-endosomal transport of PLP. To obtain further support for this conclusion, we determined the amount of PLP being released in association with exosomes after interfering with the function of the ESCRT-machinery. Knock-down of Tsg101 and Alix or expression of a dominant-negative Vps4 did not impair the secretion of PLP with exosomes (Figure 3.11). The assay was validated by showing that the secretion of GFP-tagged Gag protein of the Moloney murine leukemia virus with virus-like particles was strongly reduced by the expression of dominant-negative Vps4 (Figure 3.11; Garrus et al. (2001)). In addition the overexpression of Tsg101, which inhibits HIV-1 budding (Goila-Gaur et al., 2003), did not affect exosome release of PLP (Figure 3.12). Collectively our data shows that PLP is transferred into the lumen of endosomes and released with exosomes in an ESRCT-independent fashion and raises the question of the molecular mechanisms of this alternative pathway. 

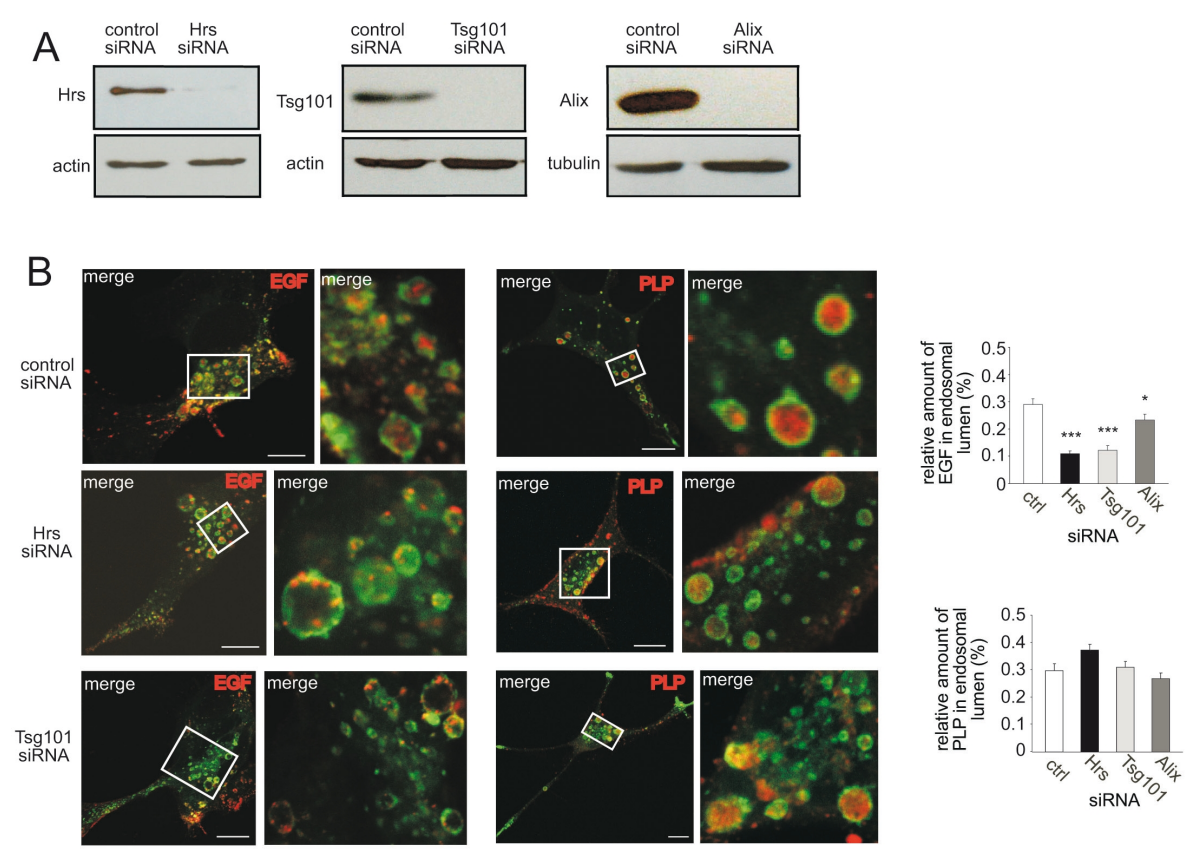

Figure 3.7: ESCRT-independent intrluminal transport of PLP. (A) A control RNA duplex or siRNA against Hrs, Tsg101 or Alix were delivered by nucleofection into Oli-neu cells. The efficiency of the knock-down was demonstrated by Western blotting (upper panel). Actin is shown as a loading control (lower panel). (B) RNAi treated cells were cotransfected with GFP-Rab5 ${ }^{\mathrm{Q79L}}$ and PLP-myc or EGFR, EGFR transfected cells were incubated for 15 min with Rhodaminelabelled EGF before fixation. Cells were then processed and analyzed for immunofluorescence microscopy. The amount of PLP or EGF in the lumen of the enlarged endosomes was quantified (fluorescence intensity in the lumen/limiting membrane). Values represent the mean $\pm \mathrm{SE}(\mathrm{n}>$ 70 endosomes; ${ }^{*} p<0.05 ;{ }^{* * *} p<0.001 ; t$ test). Scale bar, $10 \mu \mathrm{m}$. 


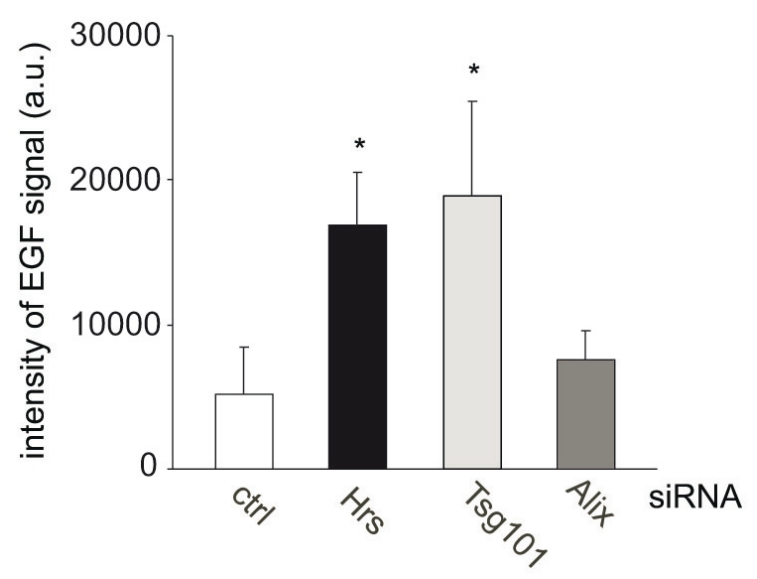

Figure 3.8: EGF degradation after treatment with siRNA against Hrs, Tsg101 and Alix. A control RNA duplex or siRNA against Hrs, Tsg101 or Alix was delivered by nucleofection into Olineu cells. Cells were transfected with EGFR-EGFP, incubated with Rhodamine-labelled EGF for $15 \mathrm{~min}$, washed, and incubated for $4 \mathrm{hr}$ in conditioned culture medium to allow EGF degradation. For quantification, images of randomly selected transfected cells were recorded and fluorescence intensities were quantified. Values represent the mean \pm SE ( $n>70$ cells; ${ }^{*} p<0.05 ; t$ test).
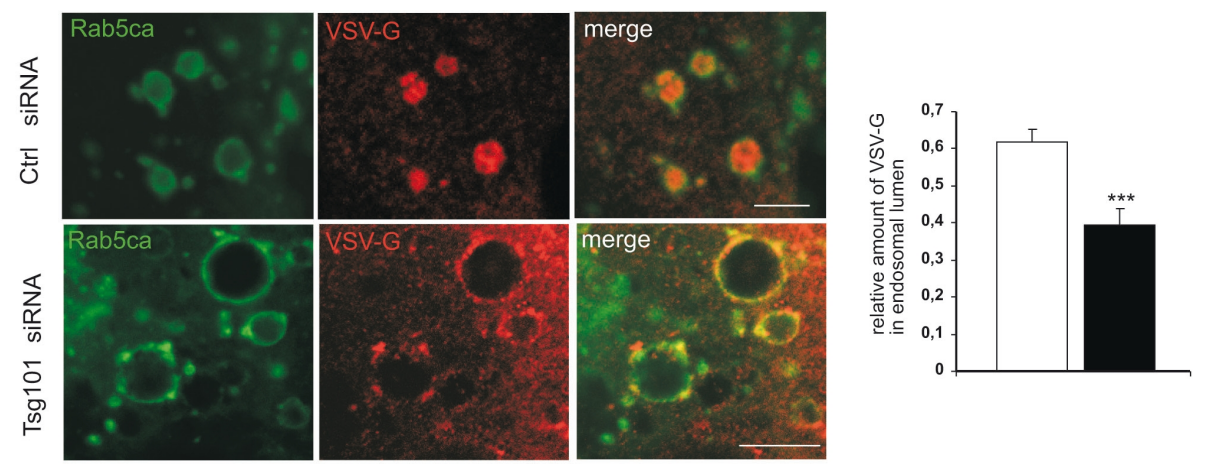

Figure 3.9: Tsg101-dependent transport of VSV-G. A control RNA duplex or siRNA against Tsg101 was delivered by nucleofection into Oli-neu cells. RNAi treated cells were cotransfected with GFP-Rab5 ${ }^{\mathrm{Q} 79 \mathrm{~L}}$ and VSV-G-myc. Cells were then processed and analyzed for immunofluorescence microscopy. The amount of VSV-G in the endosomal lumen was quantified (fluorescence intensity in the lumen/limiting membrane). Values represent the mean $\pm S E(n>40$ endosomes, ${ }^{* * *} p<0.0001 ; t$ test). Scale bar, $5 \mu \mathrm{m}$. 

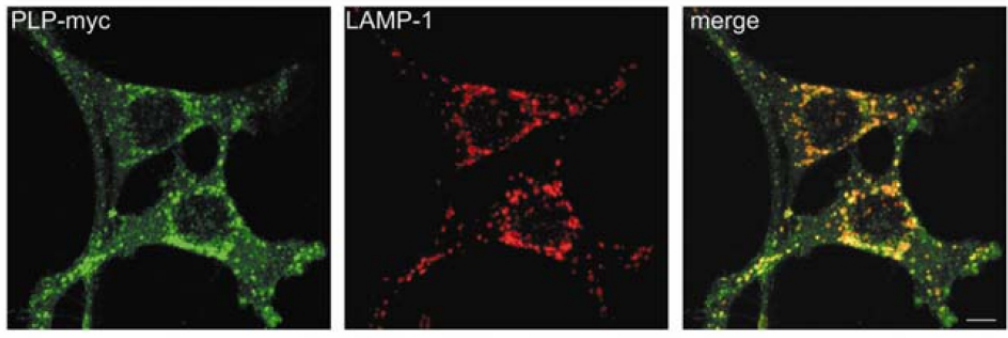

control
siRNA
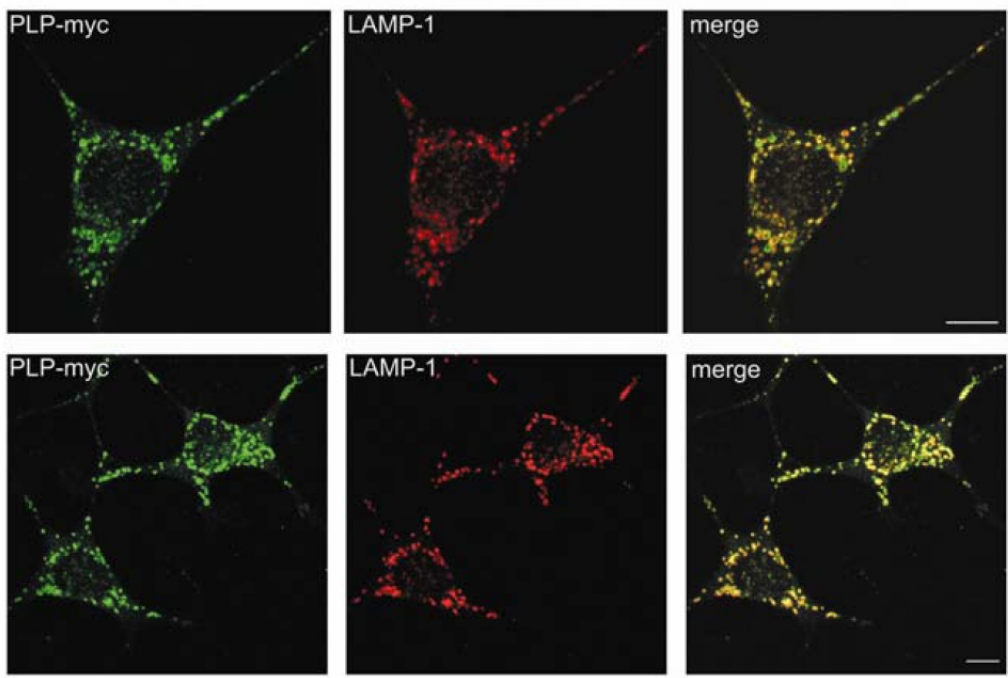

Hrs
siRNA
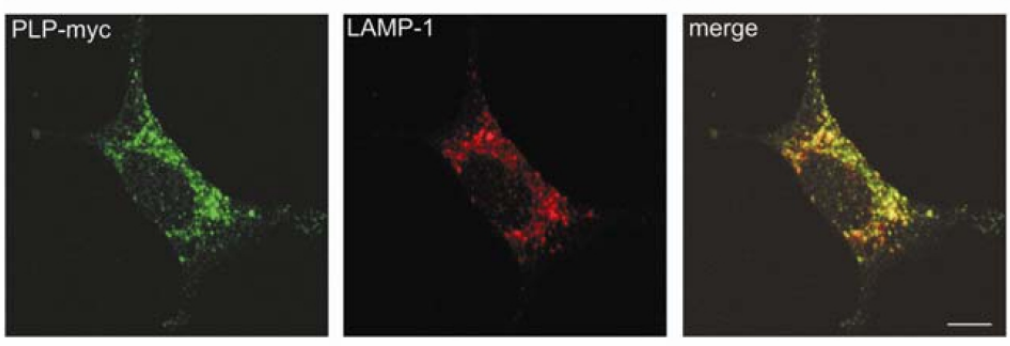

Tsg101
siRNA

Alix
siRNA

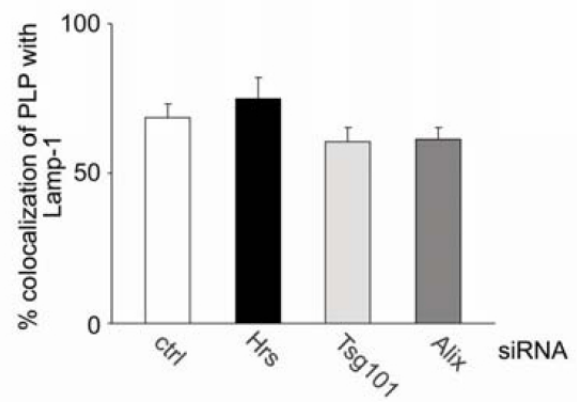

Figure 3.10: Colocalization of PLP and Lamp-1 after treatment with siRNA against Hrs, Tsg101 and Alix. A control RNA duplex or siRNA against Hrs, Tsg101 or Alix was delivered by nucleofection into Oli-neu cells. Cells were transfected with PLP-myc, processed and analyzed for immunofluorescence microscopy. Colocalization of PLP and Lamp-1 was determined. Values represent the mean \pm SE ( $n>30$ cells). Scale bar, $5 \mu$ m. 
A

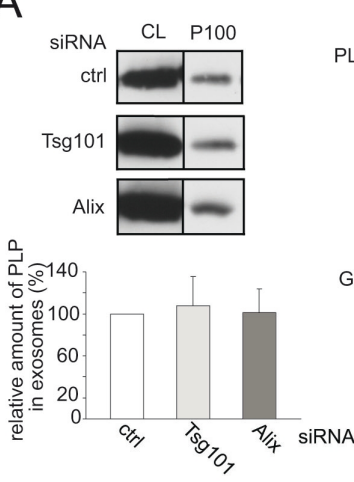

B
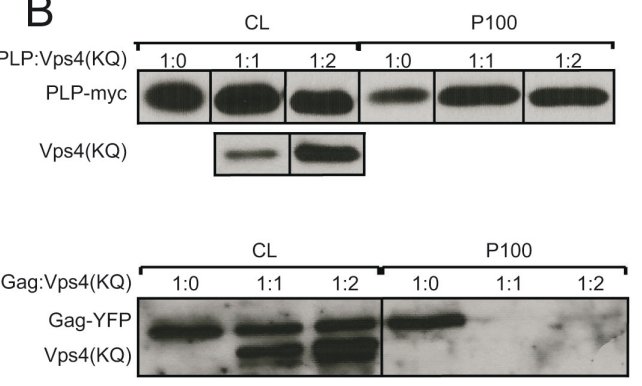
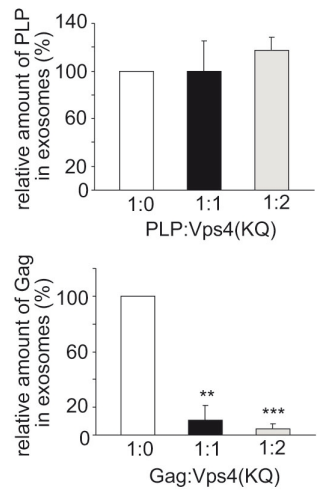

Figure 3.11: ESCRT-independent exosome release of PLP. (A) RNAi treated cells were transfected with PLP and amount of PLP was determined in the cell lysate $(C L)$ and in the $100000 \mathrm{~g}$ pellet (P100) of the sequential centrifugation steps of the culture medium. Results are expressed as the mean $\pm S D$ of five experiments. (B) Cells were cotransfected with PLP-myc or Gag-YFP and different ratios of ATPase binding defective Vps4 (KQ). The amount of Gag-YFP and PLP-myc was determined in the cell lysate (CL) and in the $100000 \mathrm{~g}$ pellet (P100). Results are expressed as the mean \pm SD of three experiments $\left({ }^{* *} p<0.01\right.$; ${ }^{* * *} p<0.001$; one sample $t$ test against $100 \%)$.
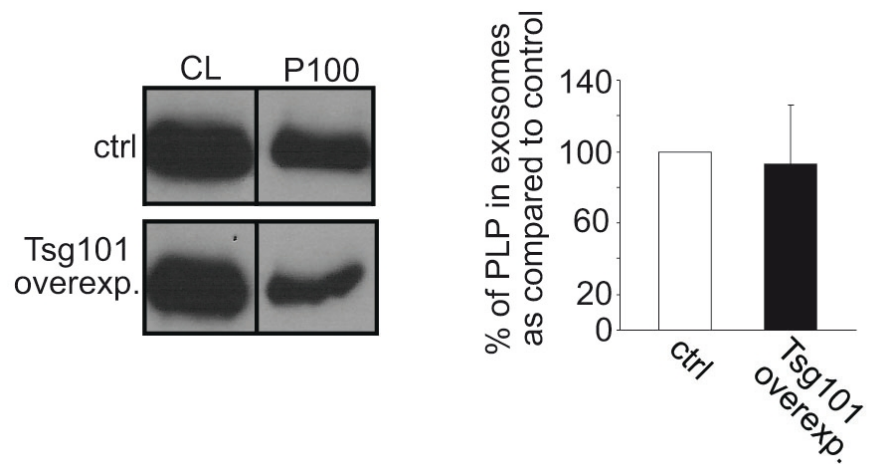

Figure 3.12: Tsg101 overexpression does not affect exosome release of PLP. Cells were transfected with PLP-myc or cotransfected with PLP-myc and Tsg101-HA. The amount of PLPmyc was determined in the cell lysate (CL) and in the $100000 \mathrm{~g}$ pellet (P100). Results are expressed as the mean $\pm S D$ of three experiments. 
A

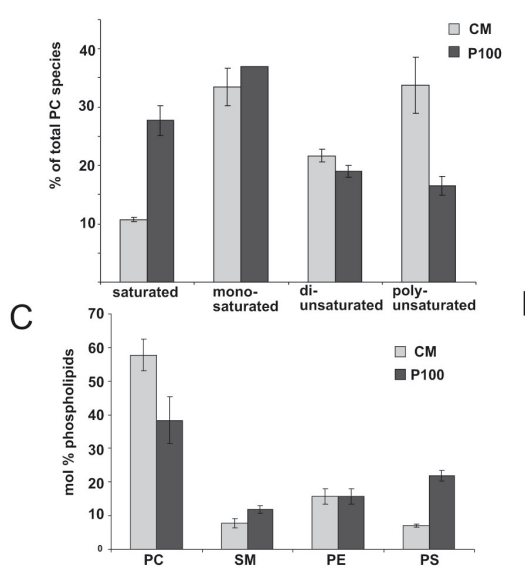

B

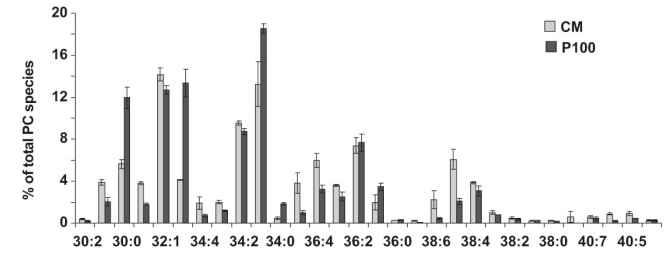

D

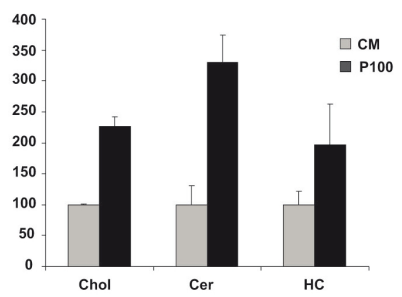

Figure 3.13: Lipid analysis of exosomes. Analysis of $(A, B)$ phosphatidylcholine $(P C)$ composition, (C) phospholipid classes, and (D) ratios of individual lipid classes from total cellular membranes (CM) and the exosome fractions (P100). The data are from three independent experiments; values are means $\pm \mathrm{SD}$. Chol, cholesterol; $\mathrm{HC}$, hexosylceramide; Cer, ceramide; PE, phosphatidylethanolamine; and PS, phosphatidylserine.

\subsubsection{A role of ceramide in the formation of exosomes}

To get insight ESCRT-independent budding machinery, we used nano-electrospray ionization tandem mass spectrometry to identify the molecular composition of exosomes. Key insights came from the lipid analysis. We found that the lipid composition was remarkably similar to the lipid composition that has been proposed for lipid rafts (de Gassart et al., 2003; Wubbolts et al., 2003). We found that exosomes were not only enriched in cholesterol, but also contained higher amounts of sphingolipids (sphingomyelin and glucocylceramide) and lower amounts of phophatidylcholine (PC) as compared to total cellular membrane (Figure 3.13). In addition, quantitative analysis of lipid subclasses revealed a striking increase in saturated PC, at the expense of polyunsaturated species in the exososmal membrane fraction.

Most importantly, we found a marked enrichment of ceramide in exosomes. In cellular membranes ceramide is formed upon the hydrolytic removal of the phosphocholine moiety of sphinogmyelin by sphingomyelinases (Clarke \& Hannun, 2006). Previous studies have shown that exogenous sphingomyelinase treatment or application of $\mathrm{C}_{6}$-ceramide can induce the formation of vesicles (Holopainen et al., 2000; Zha et al., 1998; Li et al., 1999). 

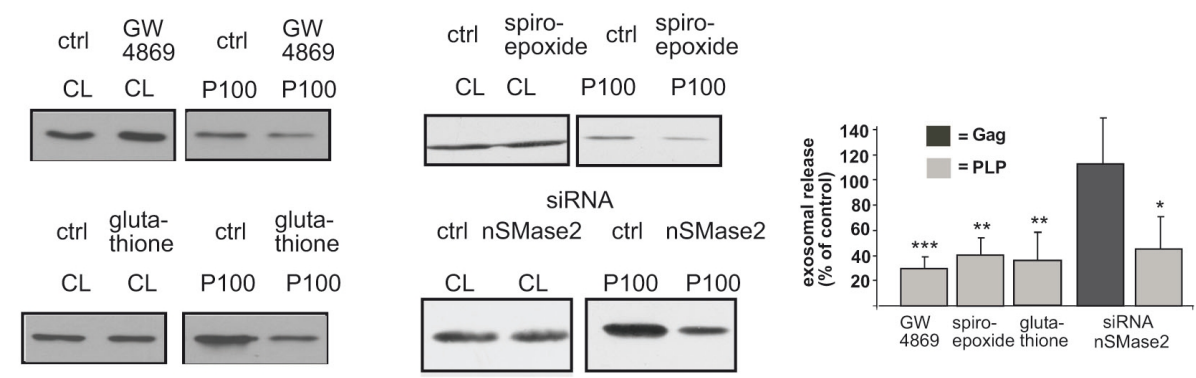

Figure 3.14: Exosomal release of PLP was reduced with inhibition of neutral sphingomyelinases. Oli-neu cells stably expressing PLP-EGFP were treated with $5 \mathrm{mM}$ GW4869, $5 \mathrm{mM}$ spiroepoxide, or $5 \mathrm{mM}$ glutathione or with the respective vehicle. nSMase2 small interfering RNA (siRNA) was delivered by nucleofection into the cells. The amount of PLP-EGFP and GagYFP was determined in the cell lysates (CL) and in the exosome fractions (P100). Results are expressed as means \pm SD of three to six experiments $\left({ }^{*} p<0.05 ;{ }^{* *} p<0.01 ;{ }^{* * *} p<0.001\right.$; one-sample $t$ test against $100 \%$ ).

To analyze the role of ceramide in exosome biogenesis, we treated Oli-neu cells stably expressing PLP-EGFP with the neutral sphingomyelinase inhibitor, GW4869. We observed a dramatic reduction of exosome release after treatment with GW4869. The effect was also observed after treatment with the two structurally unrelated neutral sphinogmyelinase inhibitors, spiroepoxide and glutathione (Figure 3.14). Furthermore, depletion of neutral sphingomyelinase 2 (nSMase2) with RNAi reduced the release of PLP with exosomes. Next, we studied the effect of neutral sphingomyelinase inhibition in intra-endosomal transport of PLP. After treatment with GW4869 a significant reduction of the amount of PLP within the endosomal lumen was observed (Figure 3.15). This was not due to an unspecific derangement of the endosomal system, as the amount of intra-endosomal VSVG remained unchanged. Furthermore, a reduction of PLP within the endosomal lumen was also observed after depletion of nSMase2 using RNAi (Figure 3.15).

To further explore the role of ceramide in the formation of intravesicular membrane, we performed experiments using giant unilamellar vesicles (GUV). We used a mixture of PC, SM and cholesterol to generate liposomes with two different lipid phases. DiD$\mathrm{C}_{18}$ and Bodipy-cholesterol were used to mark the liquid-disordered and liquid-ordered lipid phases, respectively. We exogenously added sphingomyelinase to these liposomes to analyze whether intra-vesicular membranes were formed from one of these lipid phases. 

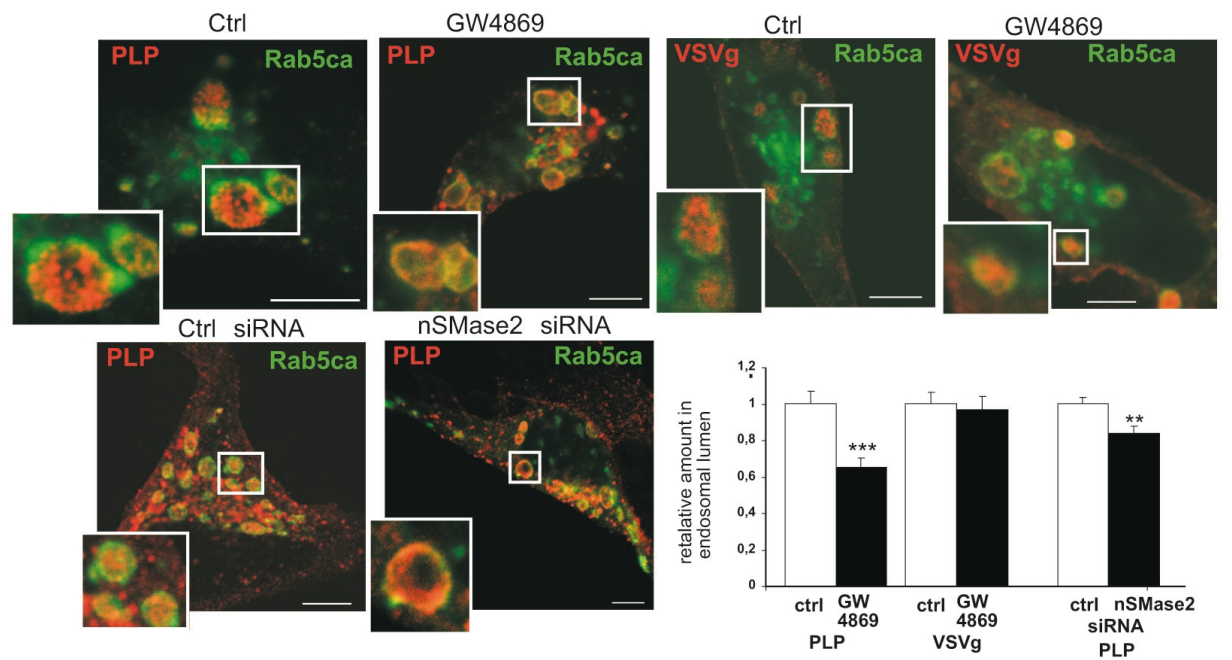

Figure 3.15: Neutral sphingomyelinase dependent intraluminal transport of PLP. Cells were treated as indicated and cotransfected with GFP-Rab5 ${ }^{\text {Q79L }}$ and PLP-myc or VSV-G and then processed and analyzed by immunofluorescence microscopy. The amount of PLP or VSV-G in the endosomal lumen was quantified (fluorescence intensity in the lumen versus the limiting membrane; normalized to 1 for the controls). Values represent means $\pm S E$ ( $n>40$ endosomes; ${ }^{* *} p<0.01,{ }^{* * *} p<0.001 ; t$ test). Scale bar, $5 \mu \mathrm{m}$. 


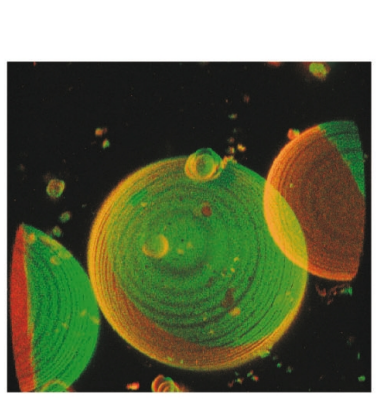

before addition

after addition

of sphingomyelinase

of sphingomyelinase
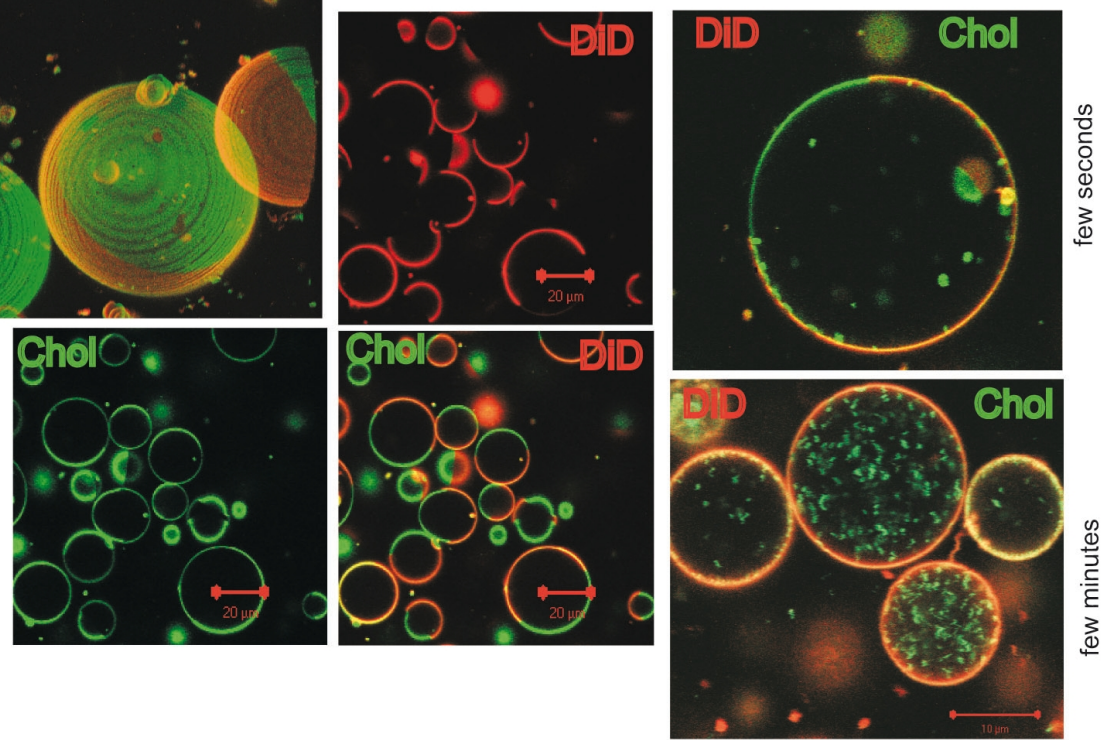

Figure 3.16: Ceramide formation triggers intraluminal budding of vesicles on GUVs. GUVs were prepared with a mixture of DOPC, SM, and cholesterol. DiD-C18 (red) and Bodipycholesterol (green) were used to mark the two lipid phases. GUVs were incubated with SMase from Staphylococcus aureus. Confocal pictures are shown before and at two different time points after the addition of SMase, as indicated.

Shortly after the addition of the sphingomyelinase, small vesicles started to bud from the liquid-ordered lipid phase and to accumulate in the lumen of the liposomes (Figure 3.16). The intravesicular membrane was predominantly labelled by bodipy-cholesterol and contained only small amounts of DiD- $\mathrm{C}_{18}$ (Figure 3.16). Similar results were observed when GM1 was included in the GUVs and fluorescently-labeled cholera-toxin was used to mark the liquid-ordered lipid phase (data not shown). 


\title{
3.2 Regulation of exosome secretion by Rab35 and its GTPase- activating proteins TBC1DA-C in oligodendrocytes
}

Most of the results in Section 3.2 have been published in:

\author{
Regulation of exosome secretion by Rab35 and its GTPase-activating proteins \\ TBC1D10A-C. \\ Chieh Hsu, Yuichi Morohashi, Shin-ichiro Yoshimura, Natalia Manrique-Hoyos, \\ SangYong Jung, Marcel A. Lauterbach, Mostafa Bakhti, Mads Grønborg, \\ Wiebke Möbius, JeongSeop Rhee, Francis A. Barr and Mikael Simons. \\ J. Cell Biol.; 189:223-232 (2010)
}

\subsubsection{Proteome analysis of Rab GTPases in exosomes}

We started our screen for Rab GTPases in exosome secretion by performing a proteome analysis of purified exosomes using liquid chromatography coupled to tandem mass spectrometry (LC-MS/MS). A total of 301 proteins were identified, of which $\sim 1 / 3$ have been previously found in exosomes from other cell types (Table A.1), confirming the purity of the preparation. Among the identified proteins was a relatively large number of Rab GTPases - Rab1a, Rab1b, Rab2a, Rab5b, Rab5c, Rab6a, Rab7, Rab8b, Rab10, Rab11b, Rab35 - many of which have previously been implicated in endosomal membrane trafficking. To analyze the relative abundance of the Rab GTPases in exosomes, we expressed all of the identified Rabs as EGFP fusion proteins in Oli-neu cells and compared the levels to PLP. As compared to PLP-EGFP, Rab proteins were found at relatively low levels in exosomes, but among the most abundant was EGFP-Rab35 (Figure 3.17).

\subsubsection{A Rab GAP screen in exosome secretion}

Based on the conserved catalytic domain, the TBC (Tre, Bub2 and Cdc16) domain, 40 Rab GAPs have been identified in the human genome. Screens to identify the target Rab proteins of the different GAPs have been performed either using in vitro GTP hydrolysis assay or yeast-two hybrid screens. However, using the known GAPs and the 60 Rabs, the Rab-GAP pairs identified form yeast-two hybrid did not always show the GTP hydrolysis activation of the Rab (Itoh et al., 2006). The Rab GAP constructs can be expressed 

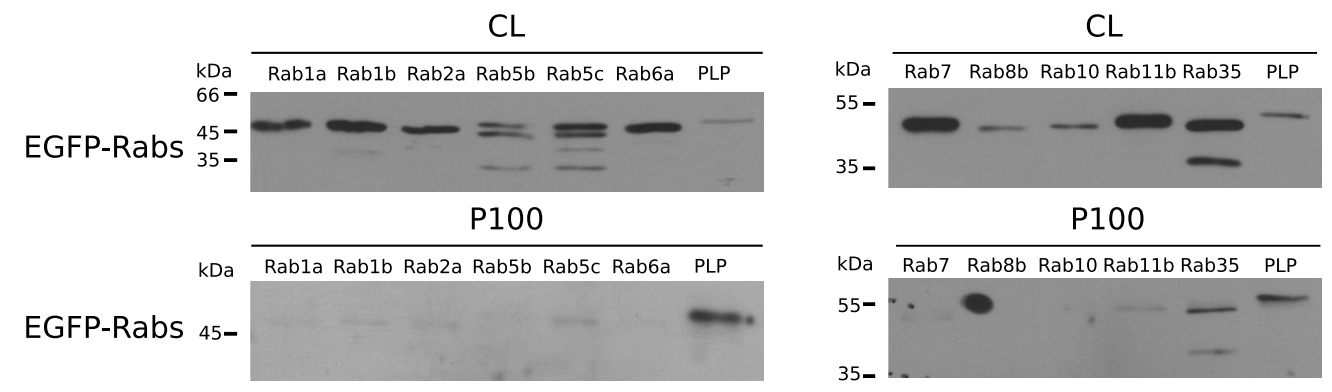

Figure 3.17: Western blot analysis of Rabs in exosomes. The Rabs identified by mass spectrometry were expressed as EGFP fusion proteins in Oli-neu cells. $16 \mathrm{hr}$ after transfection, the cells were switched to serum-free medium and the medium was collected after $\sim 4 \mathrm{hr}$ of further incubation before submitting it to sequential centrifugation steps. The amount of the EGFP-Rab proteins were determined in the cell lysates $(C L)$ and $100000 \mathrm{~g}$ pellets (P100) by Western blotting. PLP is used as reference. One representative experiment is shown.

exogenously in screening approaches. The overexpression of a Rab GAP accelerates the hydrolysis of its target Rab and shifts it to the inactive, GDP-bound state. In this fashion, Barr and colleague established a human Rab GAP library consisting 38 GFP tagged, mammalian expressing Rab GAP constructs. Using this library they have identified the interaction of RN-tre with Rab43, Rab-GAP5 with Rab5 and TBC1D20 with Rab1; and the function of the GAP was studied in various assays (Haas et al., 2005, 2007; Fuchs et al., 2007).

To define the requirement of Rab proteins in exosome biogenesis, we used this Rab GAP library to screen for the ability of each Rab GAP to reduce the secretion of PLP-EGFP in association with exosomes. Since Rab GAPs promote GTP hydrolysis of Rabs requiring a conserved catalytic domain, the TBC domain, this approach leads to the selective inactivation of the different Rab proteins (Fuchs et al., 2007; Yoshimura et al., 2007). We coexpressed PLP-EGFP with EGFP fusion proteins of all 38 predicted Rab GAPs, confirmed their expression by Western blotting with anti-GFP antibodies in the cell lysates and determined the amount of PLP-EGFP in the exosomal membrane fraction in three independent experiments. The positive candidates that reproducibly reduced the release of PLP-GFP with exosomes were re-evaluated by comparing the effects of the wild-type GAPs to that of the catalytically inactive point mutations. We found that TBC1D10B, RN-tre, TBC1D10A, TBC1D10C and TBC1D15 reduced PLP-EGFP recovery from the 

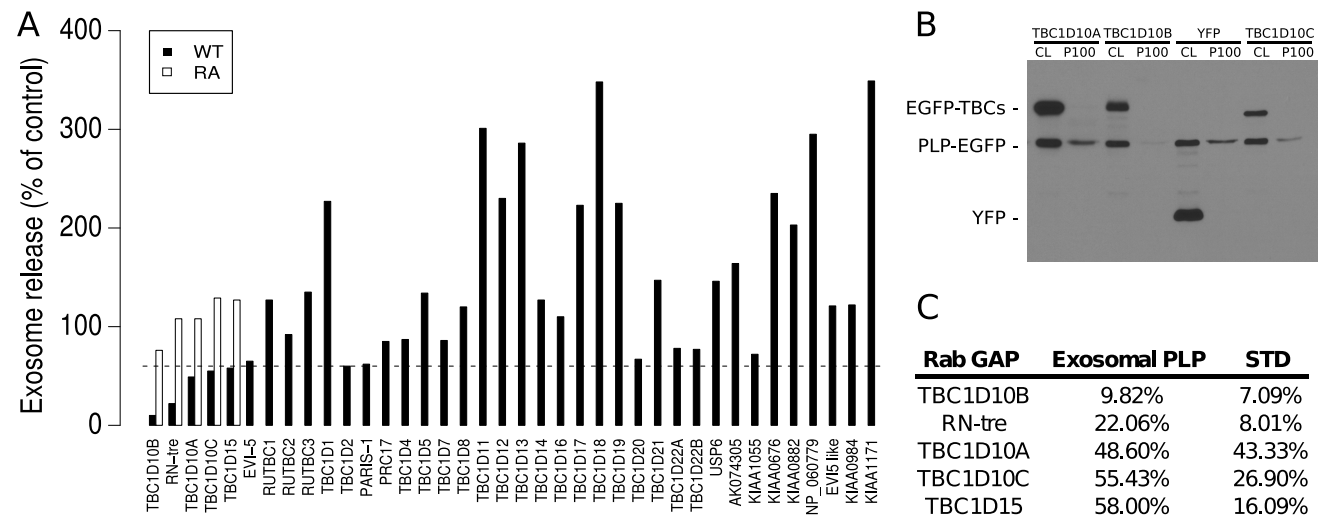

Figure 3.18: Screen of a Rab GAP library identifies the TBC1D10 family as regulators of exosome secretion. (A) PLP-EGFP was co-expressed with a library of 38 different EGFP tagged Rab GAPs in Oli-neu cells using a plasmid ratio of 2:1 (EGFP-TBC: PLP-EGFP). Cells transfected with both PLP-EFP and YFP were used as a reference. $16 \mathrm{~h}$ after transfections, the cells were switched to serum-free medium and the medium was collected after $\sim 4 \mathrm{hr}$ of further incubation before submitting it to sequential centrifugation steps. The resulting $100000 \mathrm{~g}$ pellets (exosome fraction) of each centrifugation step were analyzed by Western blotting for PLP-EGFP and the EGFP-TBCs (Rab GAPs) by anti-GFP antibodies. The average of three independent experiments is shown in the graph. Those Rab GAPs that reduced exosome release of PLP below $60 \%$ of the control, as indicated with the dotted line, were retested as catalytically inactive RA mutants (white bars). (B,C) Western blot of the cell lysates (CL) and $100000 \mathrm{~g}$ pellets (P100) is shown for one representative experiment with the TBC1D10 family and the table displays the average and the standard deviation (SD) of the five positives.

exosomal membrane fraction in a catalytically activity-dependent manner (Figure 3.18).

\subsubsection{Identification of Rab35 as the target Rab of TBC1D10A-C}

RN-tre has been reported to act on Rab5 (Lanzetti et al., 2000) and Rab43 (Haas et al., 2005), TBC1D15 on Rab7 (Zhang et al., 2005), TBC1D10A on Rab27a (Itoh \& Fukuda, 2006) and TBC1D10C on Rab35 (Patino-Lopez et al., 2008). We re-examined the effects of the TBC1D10 family towards specific Rab proteins by testing their ability to accelerate GTP hydrolysis in a biochemical assay with a wide set of Rabs (Figure 3.19). These data clearly show that all three members of the TBC1D10 family have strong and Rab35 specific GAP activity. TBC1D10B, which reduced exosome secretion most potently, showed 

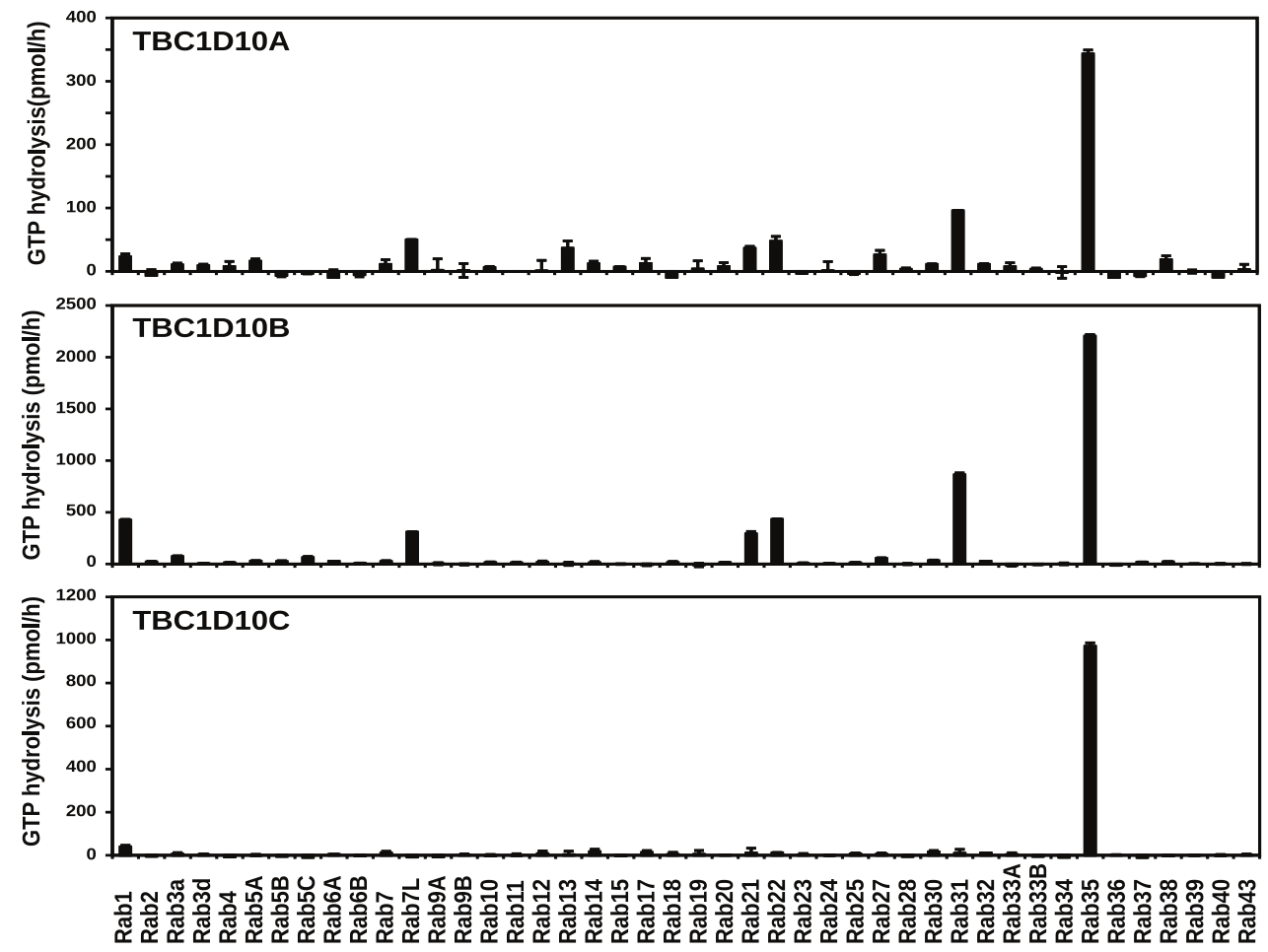

Figure 3.19: Identification of Rab35 as the target of TBC1D10A-C. Biochemical GAP assays were performed using recombinant hexahistidine-GST-tagged human Rab GTPases and hexahistidine-tagged TBC1D10 family proteins. Reactions were performed for 60 min at $37^{\circ} \mathrm{C}$, using 100 pmol GST-Rab and 10 pmol hexahistidine-tagged TBC1D10A, B, or C. GTP hydrolysis is plotted in $\mathrm{pmol} / \mathrm{h}$.

the strongest activity towards Rab35 (> $2000 \mathrm{pmol} / \mathrm{h}$ of hydrolyzed GTP, see Figure 3.19).

\subsubsection{A role for Rab35 in exosome secretion}

Since our results from the Rab GAP screen pointed to a possible role of Rab35 in the exosome pathway, we used the dominant-negative mutant of Rab35 (Rab35 ${ }^{\mathrm{N} 120 \mathrm{I}}$ ) to interfere with its function and found that it significantly decreased release of PLP with exosomes (Figure 3.20 A,D). We continued the analysis of the function of Rab35 by performing RNAi knock-down experiments. Knock-down of Rab35 by two rounds of siRNA nucleofection efficiently depleted Rab35 from the cell lysate and resulted in a significant reduction of PLP recovery from the exosomal membrane fraction (Figure 3.20 B-D) confirming the 

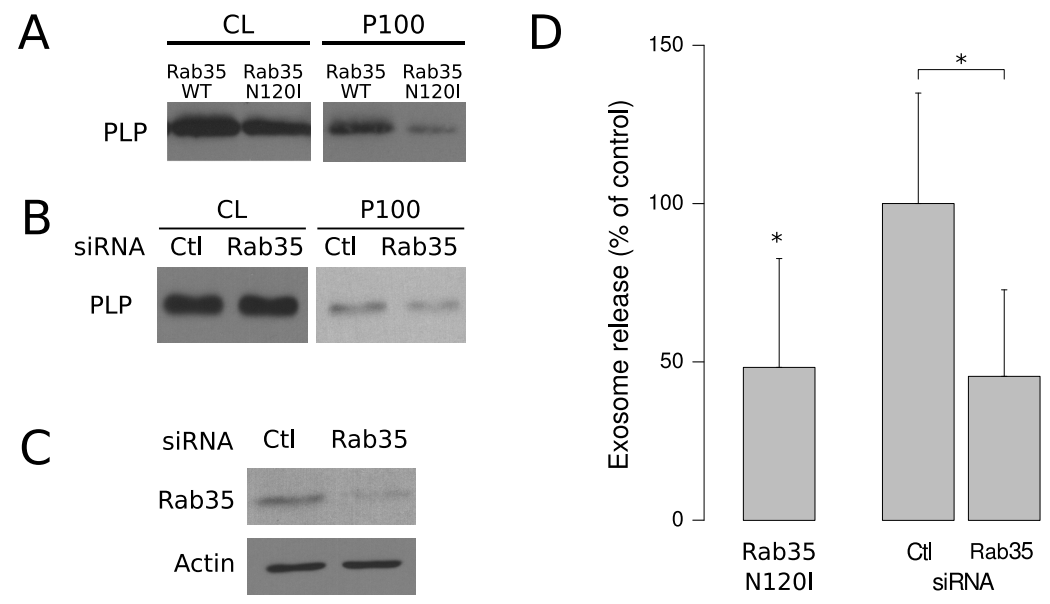

Figure 3.20: Inhibition of Rab35 function reduces exosome secretion of PLP. (A,D) Cells were transfected with PLP-myc together with either wild-type (wt) Rab35 or the dominant-negative mutant (Rab35 $\left.{ }^{\mathrm{N1201}}\right)$. The amounts of PLP in the cell lysates $(\mathrm{CL})$ and $100000 \times \mathrm{g}$ pellets (P100) were quantified. (B,C) After delivery of control (Ctl) or siRNA against Rab35 into cells by nucleofection, the cell lysates were analyzed by Western blotting with Rab35 antibodies to monitor knock-down efficiency. Actin was used as a loading control. The amount of PLP in the cell lysates (CL) and $100000 \mathrm{~g}$ pellets (P100) were determined after Rab35 RNAi. (D) Results are expressed as the mean $+\mathrm{SD}$ of four experiments $\left({ }^{*} p<0.05\right.$; one sample $t$-test; and Welch's two sample $t$ test).

findings with the dominant-negative mutant. Relatively little is known about the function of Rab35 except of its role in receptor recycling, cytokinesis and actin reorganization (Patino-Lopez et al., 2008; Kouranti et al., 2006; Sato et al., 2008; Walseng et al., 2008; Zhang et al., 2009).

\subsubsection{Localization of Rab35}

To analyze where Rab35 may act in oligodendroglial cells, we analyzed the localization of EGFP-tagged Rab35 by confocal microscopy. Wild-type Rab35, the GTP-locked Rab35 ${ }^{\mathrm{Q} 67 \mathrm{~A}}$, and its GAP, TBC1D10B, were detected at the plasma membrane, whereas the nucleotide free Rab35 $5^{\mathrm{N} 120 \mathrm{I}}$ and the GDP-locked Rab35 ${ }^{\mathrm{S} 22 \mathrm{~N}}$ were mainly found within the cytosol and in vesicles that contained PLP and Lamp-1 identifying them as late endosomes/lysosomes (Figures 3.21 and 3.22). We observed an expansion of the cell surface area following expression of either wild-type Rab35 or constitutively active GTP-locked 
Rab35 ${ }^{\mathrm{Q} 67 \mathrm{~A}}$ ((Figure 3.21).

To obtain further evidence for the plasma membrane localization of Rab35, we analyzed the expression of Rab35 in myelin, a plasma membrane extension of oligodendrocytes that is formed during the development of the central nervous system by the spiral wrapping of glial membrane around the axons (Sherman \& Brophy, 2005; Simons \& Trotter, 2007; Barres, 2008).

Indeed, we detected Rab35 at relatively high levels in the lysates of purified myelin by Western-blotting (Figure 3.23 A). Purified myelin did not contain GFAP or synaptophysin proteins specific to astrocytes and neurons confirming the purity of isolated myelin (Figure $3.23 \mathrm{~B}$ ). While the majority of myelin consists of tightly stacked membrane bilayers with no space for MVBs, a fraction of myelin is composed of more loosely organized membrane that contains larger cytoplasmic channels (e.g. paranodal loops, abaxonal space) (Salzer, 2003). We performed electron microscopy to analyze whether these structures contained MVBs. MVBs were frequently found in non-compacted regions of myelin (Figure 3.23 C, Trapp et al. (1989)) and immunoelectron microscopy revealed that MVBs contained PLP (Figure $3.23 \mathrm{C}$ ). Since the Rab35 antibody did not work reliably in immunohistochemistry, we purified non-compacted myelin from crude myelin by sucrose gradient centrifugations to determine the localization of Rab35 in myelin. Rab35 was not found in the light fraction that is enriched in compact myelin, but was recovered from the heavier fraction, which also contained contactin, a protein that localizes to the paranodal junction (Figure 3.23 A, Rios et al. (2000)).

\subsubsection{Function of Rab35}

To define the steps at which Rab35 might act, we compared the effects of the dominantnegative Rab7 and Rab35 mutants. We found that only the expression of Rab7 ${ }^{\mathrm{T} 22 \mathrm{~N}}$, but not Rab35 ${ }^{\mathrm{N} 120 \mathrm{I}}$ or Rab35 ${ }^{\mathrm{S} 22 \mathrm{~N}}$ resulted in an enlargement of PLP containing Lamp-1 positive organelles and increased the colocalization of PLP and Lamp-1 (Figures 3.24 and 3.21 ). In addition, we only observed a reduction of EGF degradation after Rab7 ${ }^{\mathrm{T} 22 \mathrm{~N}}$, but not after Rab35 ${ }^{\mathrm{N} 120 \mathrm{I}}$ expression (Figure 3.25 ) suggesting that lysosomal degradation is not regulated by Rab35. 

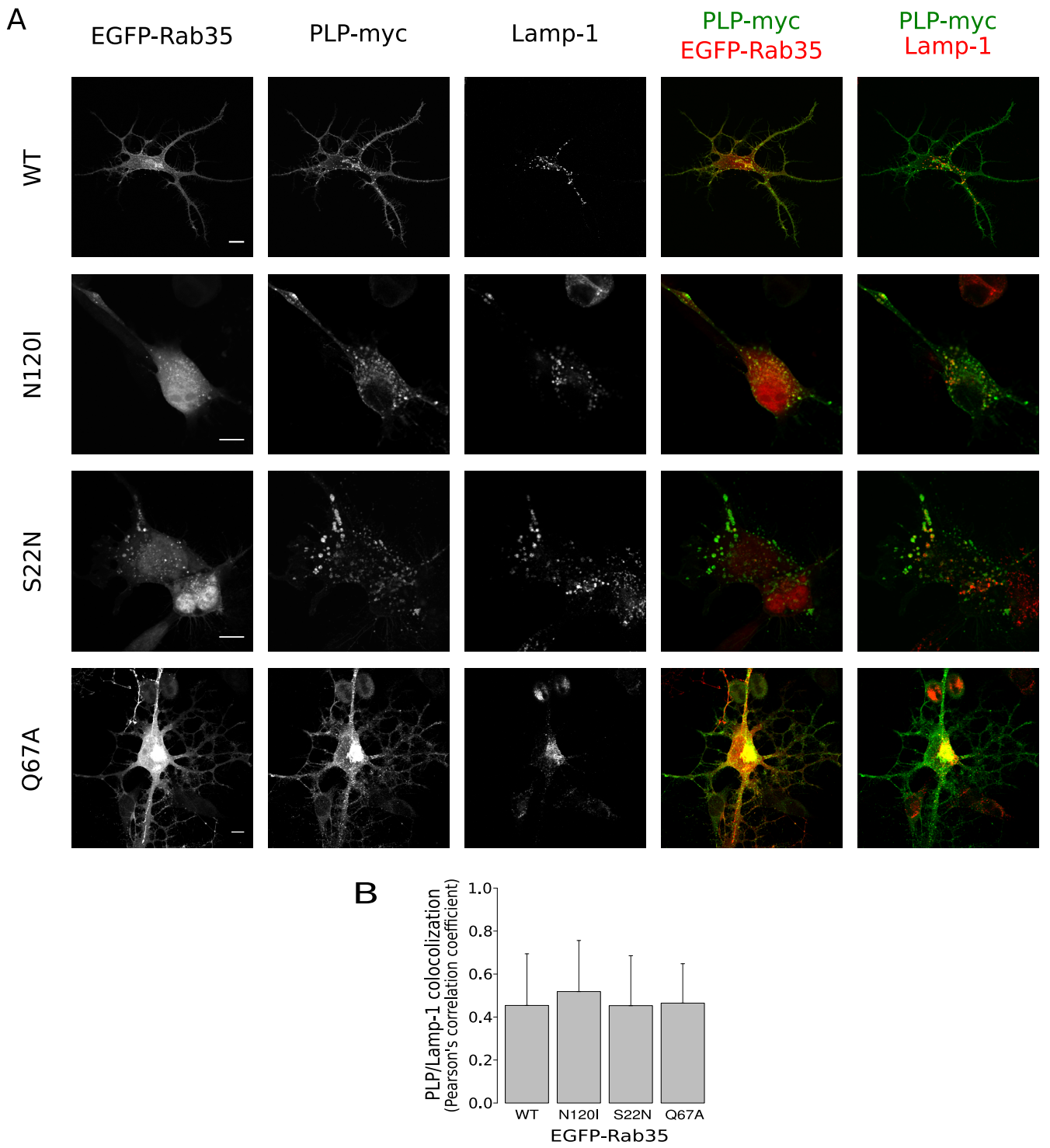

Figure 3.21: Subcellular localization of Rab35. (A) Oli-neu cells were transfected with PLP-myc together with either EGFP-Rab35 ${ }^{\mathrm{S} 22 \mathrm{~N}}$, EGFP-Rab35 ${ }^{\mathrm{N} 1201}$, EGFP-Rab35 ${ }^{\mathrm{Q} 67 \mathrm{~A}}$ or wildtype EGFP-Rab35 (wt) and analyzed by confocal microscopy. Wild-type and the GTP-locked Rab35 ${ }^{\mathrm{Q} 67 \mathrm{~A}}$ were detected at the plasma membrane, whereas the GDP-locked Rab35 ${ }^{\mathrm{S} 22 \mathrm{~N}}$ and the nucleotide-empty EGFP-Rab35 ${ }^{\mathrm{N} 1201}$ were mainly found within the cytosol and in vesicles that contained PLP and Lamp-1. Scale bar, $10 \mu \mathrm{m}$. (B) Quantification of colocalization of PLP with Lamp-1 within a $7 \times 7 \mu \mathrm{m}$ intracellular regions after co-expression with the different proteins Rab35 mutants is shown $(n=\sim 27-38)$. The values represent the mean + SD. 


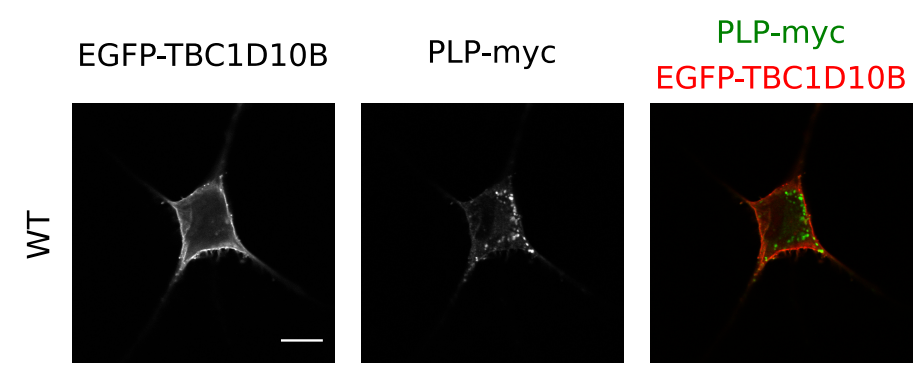

Figure 3.22: TBC1D10B localizes to the plasma membrane. Oli-neu cells were co-transfected with EGFP-TBC1D10B and PLP-myc and analyzed by confocal microscopy. Scale bar, $10 \mu \mathrm{m}$.
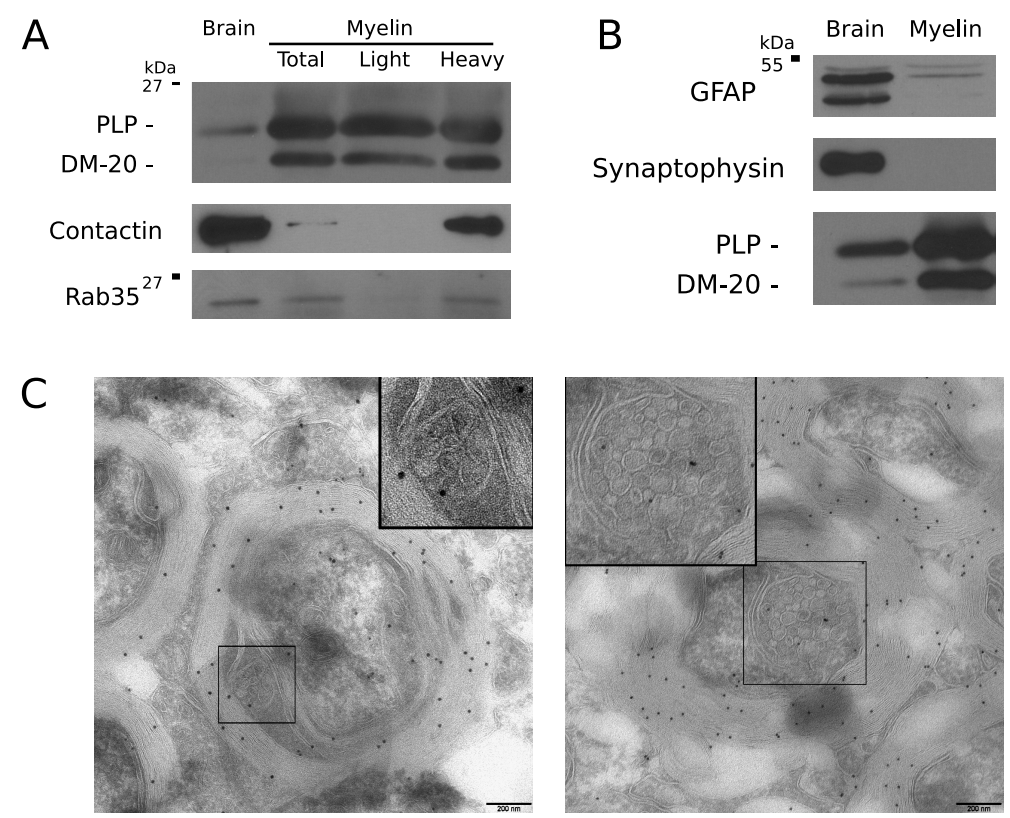

Figure 3.23: Rab35 and multivesicular structures in myelin. (A) Myelin (total) and myelin subfractions (light and heavy) were purified from the brain homogenates (brain) of adult mice and the amounts of Rab35, PLP/DM20 and contactin were determined by Western blotting. Rab35 was detected in purified myelin (total) and in the subfraction of higher density (heavy). (B) Purified myelin did not contain relevant levels of GFAP or synaptophysin proteins confirming the purity of isolated myelin. (C) Immunoelectron microscopy analysis of myelin in the spinal cord of adult mice with PLP 21 antibodies. The left image shows a MVB within a cytoplasmic channel of compact myelin and the right image shows a MVB in the abaxonal space. Scale bar, $200 \mathrm{~nm}$. 


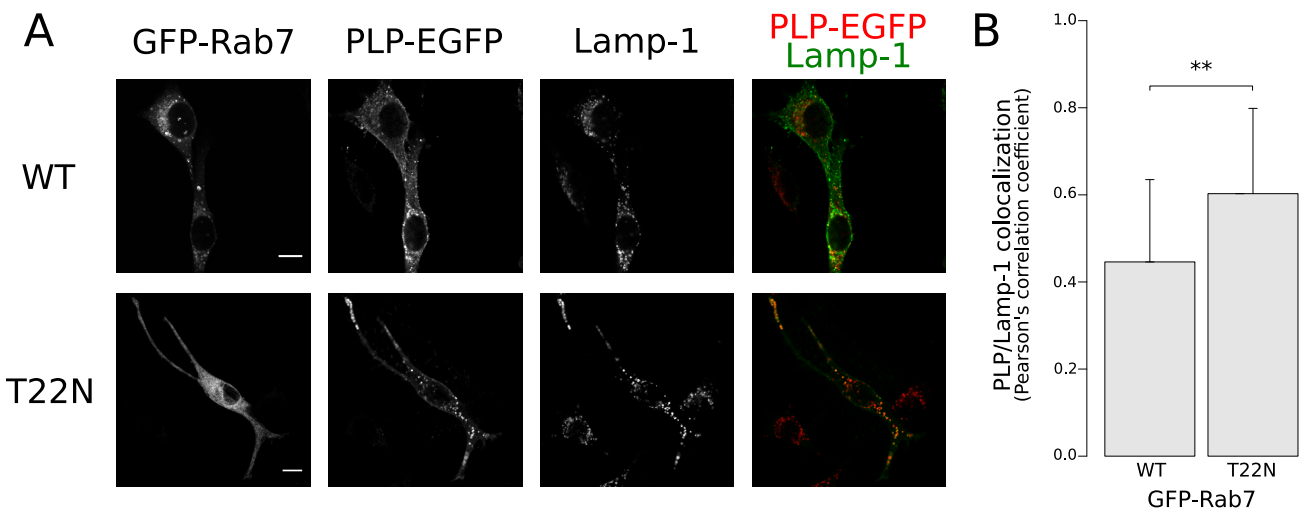

Figure 3.24: $\operatorname{Rab7}^{\mathrm{T22N}}$ changed the colocalization of PLP and Lamp-1. (A) Oli-neu cells were co-transfected with EGFP-Rab7 ${ }^{T 22 N}$ or wild-type GFP-Rab7 (wt) and PLP-myc and analyzed by confocal microscopy. Scale bar, $10 \mu \mathrm{m}$. (B) Quantification of colocalization of PLP and Lamp-1 is shown ( $n=25$ and 22). The values represent the mean + SD. ${ }^{* *}, 0.001<p<0.01$, Welch's two sample $t$-test.

Figure 3.25: Inhibition of Rab7, but not Rab35 inhibits EGF degradation. Cells were transfected with EGFP-Rab7 ${ }^{\text {T22N }}$ or EGFP-Rab35 ${ }^{\mathrm{N} 1201}$ or the respective wildtype constructs together with EGFR-EGFP, incubated with Rhodamine-labelled EGF for $15 \mathrm{~min}$, washed, and incubated for $4 \mathrm{hr}$ in conditioned culture medium to allow EGF degradation. For quantification, images of randomly selected transfected cells were recorded and 23 fluorescence intensities were quantified. Values represent the mean + SD $\left(n=54-63\right.$ cells; ${ }^{* * *} p ; 0.001$; Welch's two sample $t$ test).

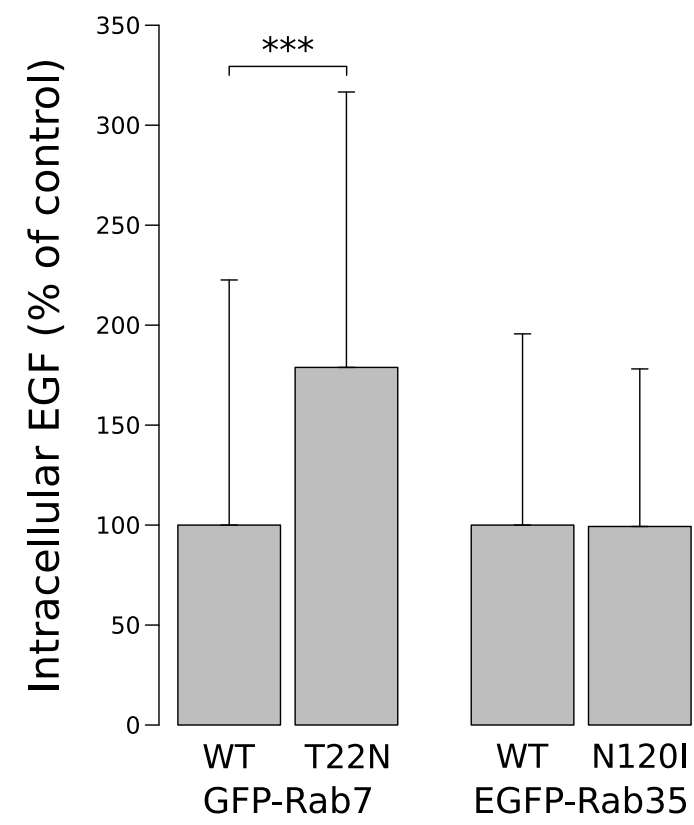




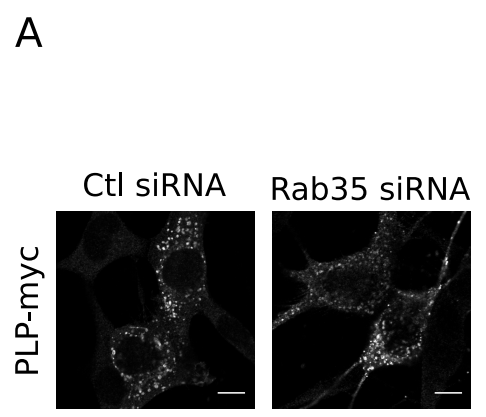

B
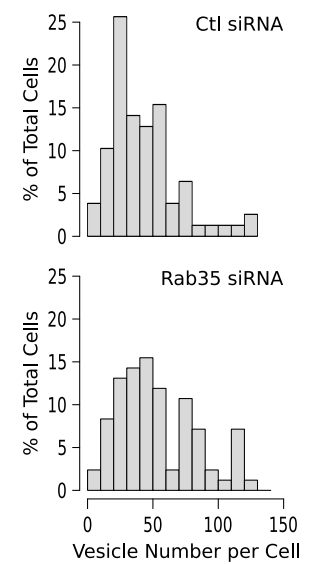
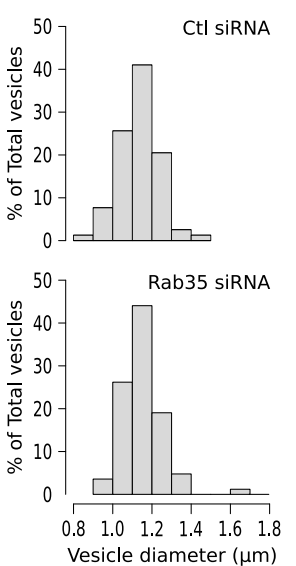

Figure 3.26: Knock-down of Rab35 expression accumulated PLP containing endosomes. (A) Control (Ctl) or siRNA against Rab35 were delivered into cells by nucleofection and the cells were imaged by immunofluorescence microscopy to detect PLP-myc. Scale bar, $10 \mu \mathrm{m}$. (B) Vesicle number and size are displayed in a histogram. Note that vesicle size is unaffected, whereas the number of vesicles increased after Rab35 knock-down ( $n=\sim 80$ cells from 3 independent experiments).

In order to determine how Rab35 functions, we performed RNAi experiments to deplete Rab35 and analyzed the number and size of the PLP-containing intracellular vesicles. While vesicle size was unaffected, the total number of vesicles increased by $\sim 35 \%$ after Rab35 knock-down (Figure 3.26). These vesicles were identified as late endosomes/lysosomes based on their colocalization with Lamp-1.

The intracellular accumulation of PLP in Lamp-1 positive vesicles and the reduction in exosome secretion after Rab35 depletion suggests that a pathway from the endosomal system to the plasma membrane is regulated by Rab35.

To analyze the possibility that Rab35 controls vesicular movement, time-lapse experiments were performed using LysoTracker as a probe to identify PLP/Lamp-1 containing endosomes (Trajkovic et al., 2006). The effects of the constitutive active and inactive mutants of Rab35 were compared in their ability to influence the mobility of endosomes. We used a recently developed algorithm to indentify and track individual vesicles automatically in a video sequence (Westphal et al., 2008). Data from 47 movies from 3 independent experiments were pooled to provide a quantitative assessment of vesicle motility. Surprisingly, we find that active GTP-locked Rab35 ${ }^{\mathrm{Q} 67 \mathrm{~A}}$ slightly reduces the motility of LysoTracker 

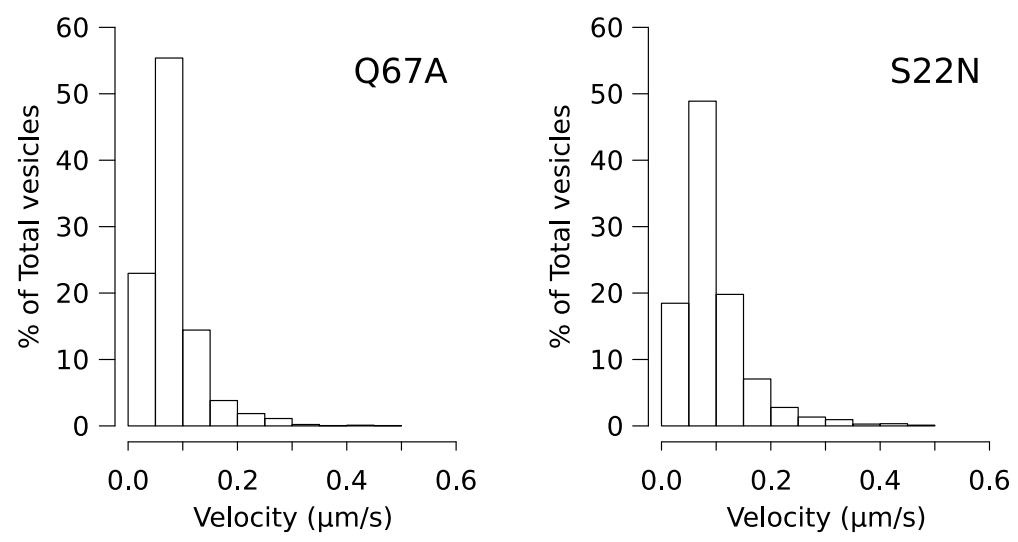

Figure 3.27: Rab35 ${ }^{\mathrm{Q67A}}$ reduced the mobility of endosomes. To analyze vesicular movement, PLP-myc was cotransfected with wild-type or mutant EGFP tagged Rab35, cells were stained with LysoTracker Red DND-99 and time-lapse images were acquired at 1 frame every $2 \mathrm{sec}$ at $37^{\circ} \mathrm{C}$. The motility of LysoTracker labelled vesicles was slightly reduced when active GTP-locked Rab35 ${ }^{\mathrm{Q} 7 \mathrm{~A}}$ was expressed ( $\mathrm{n}=\sim 1800$ vesicles from 3 independent experiments).

labelled vesicles (by $14.6 \% \pm 3.8 \%$ as compared to Rab35 ${ }^{\mathrm{S} 22 \mathrm{~N}} ; \mathrm{n}=\sim 1800$ vesicles; $p<$ 0.001, Welch's two sample $t$ test; Figure 3.27). One possible explanation is that Rab35 Q67A promotes vesicle docking/tethering. To test this possibility, cells were imaged with total internal reflection fluorescence microscopy (TIRF) to observe the vesicles just beneath the plasma membrane. We, indeed, find that that Rab35 ${ }^{\mathrm{Q} 67 \mathrm{~A}}$ increased the number of LysoTracker labelled vesicles within the evanescent excitation field $(\sim 120 \mathrm{~nm})$ by around $50 \%$ as compared to GDP-locked Rab35 ${ }^{\mathrm{S} 22 \mathrm{~N}}$ (Figure $3.28 \mathrm{~B}$ ). To determine whether these vesicles were docked/tethered to the plasma membrane we performed time-lapse TIRF experiments. To estimate vesicle motility the temporal colocalization was determined by calculating the Pearson's correlation coefficient of pairs of images separated by time (Huang et al., 2007). In such an analysis, the degree of temporal colocalization is inversely related to vesicle motility. Expression of Rab35 ${ }^{\mathrm{Q} 67 \mathrm{~A}}$ increased vesicle immobilization as compared to the inactive form of Rab35 (Figure $3.28 \mathrm{C}$ ).

\subsubsection{GTPase-defective Arf6 ${ }^{\mathrm{Q} 6 \mathrm{~L}}$ reduces exosome secretion}

Previously, Rab35 has been shown to localize to phosphatidylinositol 4,5-bisphosphate (PIP2) enriched intercellular bridges during cytokinesis (Kouranti et al., 2006). Importantly, PIP2 accumulation in the cleavage furrow was prevented by the expression of 

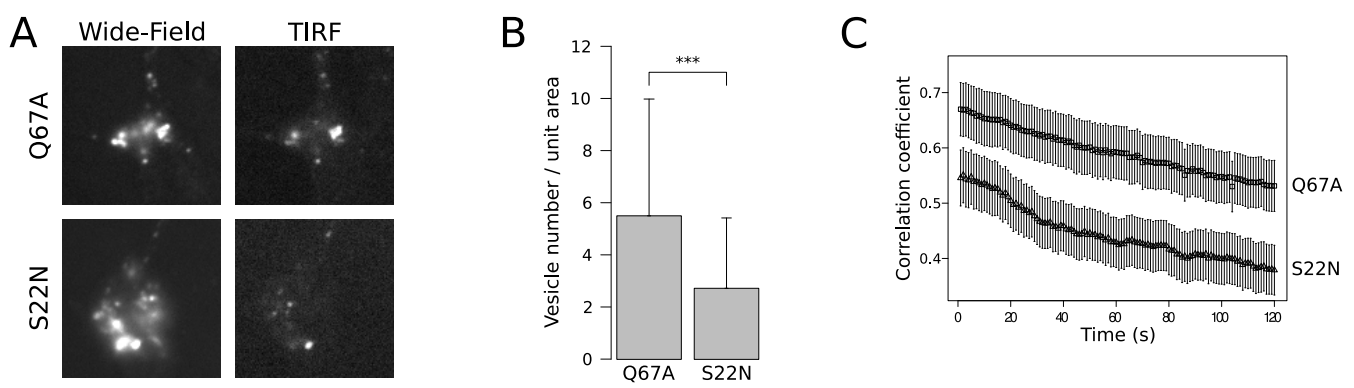

Figure 3.28: Rab35 regulated endosome attachment to the plasma membrane. (A,B) The average number of LysoTracker Green DND-26 labelled vesicles in a field of $12.7 \mu \mathrm{m} \times 12.7 \mu \mathrm{m}$ (unit area) was determined in the evanescent excitation field. Expression of the GTP-locked Rab35 ${ }^{\mathrm{Q67A}}$ increased the number of vesicles in the TIRF evanescence field as compared to the GDPlocked Rab35 ${ }^{\mathrm{S} 22 \mathrm{~N}}$ ( $\mathrm{n}=70$ cells from 3 independent experiments; ${ }^{* \star *} p<0.001$; Welch's two sample $t$ test; mean $+\mathrm{SD}$ ). Scale bar, $5 \mu \mathrm{m}$. (C) The mobility of LysoTracker Green DND-26 labelled vesicles was determined by time-lapse TIRF microscopy and analyzed by calculating the temporal correlation coefficient between pairs of images separated by time. The degree of temporal colocalization is inversely related to vesicle motility $(n=\sim 35$ videos from 3 independent experiments; mean \pm SEM).

dominant negative Rab35 (Kouranti et al., 2006). Since PIP2 is an important cofactor for exocytosis, we also analyzed whether redistributing PIP2 from the plasma membrane affected exosome secretion. To this end, the constitutively active GTPase-defective Arf6 ${ }^{\text {Q67L }}$ mutant was expressed in Oli-neu cells, which led to the formation of large intracellular PIP2-enriched vacuoles as previously described in other cell types (data not shown) (Brown et al., 2001). Interestingly, these vacuoles also contained Rab35 (Figure 3.29. When GTPase-deficient Arf6 ${ }^{\mathrm{Q} 67 \mathrm{~L}}$ was co-expressed with PLP, less PLP was released in association with exosomes (Figure 3.29). These data are reminiscent of $\mathrm{Ca}^{2+}$-dependent release of dense-core vesicles in neurons, which is blocked by $\operatorname{Arf}^{6 \mathrm{Q} 67 \mathrm{~L}}$ (Aikawa \& Martin, 2003). At this time, the exact role of Rab35 in this process remains unclear, but one possible interpretation is that Rab35 acts together with Arf6 in the regulation of PIP2 levels for exocytosis events at the plasma membrane. Another possibility is that Rab35 recruits docking or tethering factors at the plasma membrane and requires PIP2 for its targeting or function. 

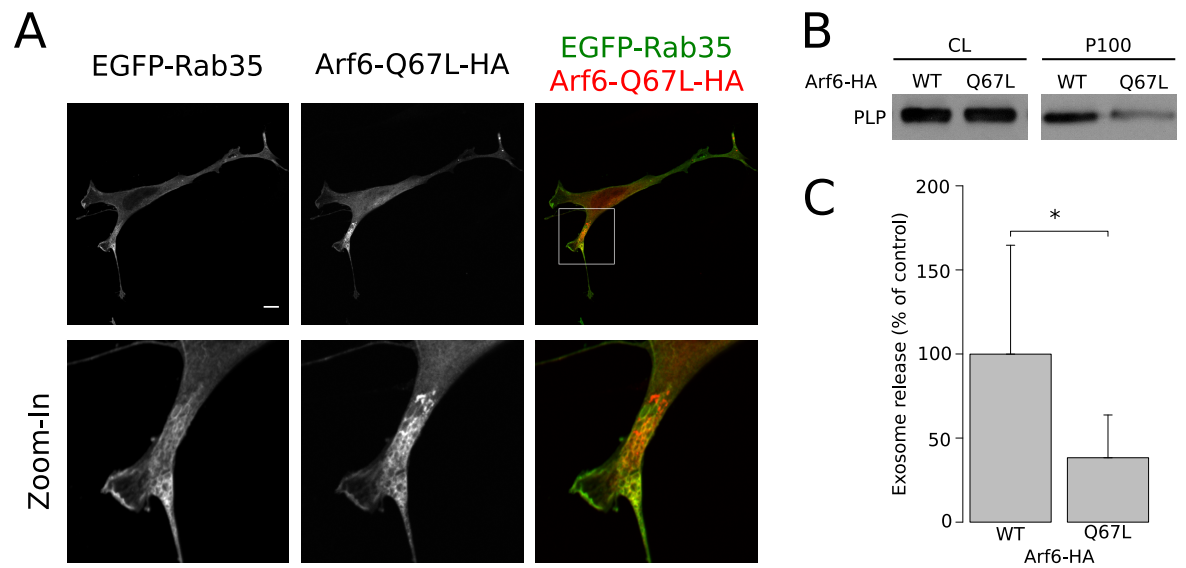

Figure 3.29: Effect of Arf6 ${ }^{067 L}$ on PLP localization and exosome release. (A) Cells were cotransfected with EGFP-Rab35 and Arf6 ${ }^{\mathrm{Q} 67 \mathrm{~L}}$-HA processed and analyzed by confocal microscopy. The zoom-in shows the localization of EGFP-Rab35 to vacuoles formed by Arf6 ${ }^{\text {Q67L-HA. (B) Cells }}$ were transfected with PLP-myc and with either wild-type (WT) Arf6-HA or the constitutively active GTPase-defective Arf6 ${ }^{\mathrm{QQ67L}}$-HA. The amount of PLP in the cell lysates $(\mathrm{CL})$ and $100000 \mathrm{~g}$ pellets (P100) were determined and quantified. (C) Results are expressed as the mean + SD of four independent experiments $\left({ }^{*} p<0.05\right.$, Welch's two sample $t$ test). 


\section{Chapter 4}

\section{Discussion}

\subsection{Mechansims of exosome biogenesis}

\subsubsection{ESCRT-independent release of exsomes}

Using an oligodendroglial cell line that contains large quantities of multivesicular endosomes, we have addressed the question of how exosomes are formed.

Exosomes are thought to originate from the release of ILV as a consequence of fusion of MVBs with plasma membrane. Some cells shed larger membrane structures from plasma membrane that can be purified from cell cultural medium, such as protasomes like granules from prostate cell (Nilsson et al., 1999) and ectosomes from neutrophils (Hess et al., 1999). To address whether vesicles released from Oli-neu cells derive from the endosomal system, we expressed the the Rab5 ${ }^{\mathrm{Q} 79 \mathrm{~L}}$ mutant to trap the transport of PLP within the endosomal system. This treatment led to the expected enlargement of the endosomes Rab5 ${ }^{\mathrm{Q} 79 \mathrm{~L}}$. By electrom microscopy we find that the enlarged endosomes are multivesicular and accumulates PLP in ILVs. In addition, we did an extensive biochemical and morphological analysis of the exosomes released by oliogodendroglial cells. The exosomes had the expected size, cup-shaped morphology and density (Figure 3.2). Furthermore, several exogenously expressed membrane proteins, such as VSVG, EGFR and APP, were found only in little amount, if at all, in our exosomal preparations (Figure 3.3), showing that the contamination from the other membrane sources was minimal. Our proteome analysis of the exosomes revealed that they contain a large number of proteins that have previously been described in exosomes (Table A.1). Based on these findings, we are confident that our biochemical purification protocol allows the isolation of relatively pure population of 
exosomes without major contaminations.

Using this model system, we addressed the question of how these exosomes are generated.

In general, the formation of ILVs involves the lateral segregation of the cargo with in the limiting membrane of an endosome, followed by the generation of an inwardly budding vesicle and the fission of the vesicle into the interior of the endosomes. While the ESCRT proteins are required for the targeting of membrane for lysosomal degradation, the function of the ESCRT machinery in the formation of ILVs that are further secreted as exosomes is less clear. Proteomic analyses of purified exosomes from various cell types showed an enrichment of some ESCRT components and ubiquitinated proteins (Simpson et al., 2008). These observations lead to the hypothesis that ESCRT function could be required for sorting of exosomal cargo.

However, we show here that the release of PLP in association with exosomes occurs in ESCRT-independent manner. Neither the expression of a dominant-negative version of VPS4 or the knockdown of Alix or TSG101 interfered with the release of PLP with exosomes (Figure 3.11).

As a control, we studied the release of virus-like particles (VLPs) formation by expressing the Gag protein from the retrovirus, murine leukemia virus (MuLV). Gag induced VLP formation has similar physical properties as exosomes, such as the size (with diameter of 100-120 nm, Gheysen et al. (1989)) and density (1.14-1.19 g/ $\mathrm{cm}^{3}$, Karacostas et al. (1989); Notka et al. (1999)) and are usually collected from the medium with centrifugation at $90000 \mathrm{~g}$ through a $20 \%(0.58 \mathrm{M})$ sucrose cushion. Indeed, blocking the function of the ESCRT machinery led to the inhibition of VLP formation, but not the secretion of PLP with exosomes (Figures 3.11 and 3.12). These results established that the release of PLP occurs in an ESCRT-independent manner. They are consistent with the findings that release of several membrane proteins requires high-order oligomerization, but not the function of ESCRT (Fang et al., 2007). There is more evidence the clustering of exosomal cargo is an important sorting determinant. For example, antibody-induced crosslinking of the transferrin receptor in reticulocytes or the MHC-II in lymphocytes enhances their release with exosomes (Vidal et al., 1997; Muntasell et al., 2007). The oligomerization of exosomal components may lead to recruitment and stabilization of exosomal membrane 
domains followed by its budding and fission at the domain boundaries.

\subsubsection{Ceramide in exosome biogenesis}

\section{How are these domains formed?}

To study possible domain formation in endosomes, we used GTP-locked Rab5 ${ }^{\mathrm{Q} 79 \mathrm{~L}}$ to enhance the early endosomal fusion and to generate enlarged endosomes (Stenmark et al., 1994). Because of the size of Rab5 ${ }^{\text {Q79L }}$ enlarged endosomes, the suborganelle localization can be analyzed with fluorescent confocal microscopy. We find that PLP is trapped in these enlarged endosomes and indeed concentrates in domains that clearly separate from domains that contain the EGFR. PLP also separates from Hrs, an ESCRT component (Figure 3.6). Instead, we observed that PLP colocalizes with a GPI-anchored protein and Flotillin-2, which are known to localize to lipid-rafts (Figure 3.6, Brown \& London (2000); Salzer \& Prohaska (2001)).

These results led to the hypothesis that specific lipids might be required to form an exosomes. To test this we purified exosomes and determined their lipid composition. We find that exosomes are enriched in the lipid ceramide (Figure 3.13). Inhibition of ceramide generation, using different pharmacological inhibitors of neutral sphingomyelinases or direct depletion of neutral sphingomyelinase 2 reduces exosome secretion (Figures 3.14). How can these results be explained?

The local generation of ceramide may promote vesicle formation by modifying the physical properties of the membrane. Ceramide has many physical properties that may be important to generate a vesicle. Ceramides are cone-shaped with small polar head groups. The cone-shape structure induces negative curvature in the membrane, thereby promoting membrane budding and possibly membrane fission.

When mixed into model membranes or even in cellular membrane, ceramides induce lateral phase separation and domain formation (Cremesti et al., 2002). It is therefore possible that the enzymatic generation of ceramide in the one leaflet of the membrane might be sufficient to induce the budding a membrane domain. This might be due to a process that is referred to as domain-induced budding (Baumgart et al., 2003). Domain-induced 
budding takes place when a domain grows and becomes unstable at a certain size. The budding occurs by competition between the bending energy of the domain and the line tension of the domain edges.

Consequently, the function of the ESCRT-machinery to deform the membrane does not seem to be required in this pathway. There are some examples of proteins that may use such a pathway such as the melanosomal protein (Pmel17) that requires a lumenal domain-dependent and an ESCRT-independent pathway (Theos et al., 2006).

Furthermore, proteins, such as tetraspanins, may partition into ceramide-induced domains. Tetraspanins have the capacity to form oligomers and so called tetraspanin webs by interacting with other tetraspanins (Boucheix \& Rubinstein, 2001). These protein-protein interaction might also be required for domain formation in a biological membrane.

\section{How is ceramide formed in a cell?}

Sphingomyelinases (SMases) are the enzymes which hydrolyze sphingomyelin into ceramide and phosphocholine (EC 3.1.4.12). According to their optimal $\mathrm{pH}$ for activity, they are classified into alkaline, neutral and acid SMases. In mammals, the alkaline SMase is found in the digestive system (Duan, 2006), whereas the two isoforms of acid SMase, the lysosomal acid SMase and the secreted acid SMase, function in the degradation and signaling of sphingomyelin both within the cell and in the extracellular space (Schuchman et al., 1991; Utermöhlen et al., 2008; Pavoine \& Pecker, 2009). The SMases that trigger ceramide formation in the intraluminal pathway of an endosome are is most likely the neutral SMases (nSMases). The inhibition of inward budding of PLP signal into the lumen of the Rab5 ${ }^{\mathrm{Q79L}}$ enlarged endosomes and the inhibition of exosome secretiona after the treatment with nSMase inhibitors point to the involvement of nSMases in this process. (Figures 3.14 and 3.15).

Three nSMases have been identified. NSMase1 has no in vivo SMase activity (Zumbansen \& Stoffel, 2002); nSMase2 is found mainly in the brain (Hofmann et al., 2000); nSMase3 is predominantly expressed in heart and skeletal muscle (Krut et al., 2006). NSMase2 might be the nSMase which hydrolyse the sphingomyelin on the cytosolic leaflet of the endosomal membrane and mediate the transport of PLP into ILVs and exosomes. Indeed 
RNAi against nSMase2 reduced the release of PLP with exosomes (Figures 3.14 and 3.15).

NSMase2 is a integral membrane protein with two hydrophobic membrane domains and multiple palmitoylation sites which regulate its subcellular localization (Tani \& Hannun, 2007a,b). Tumor necrosis factor-alpha $(\mathrm{TNF}-\alpha)$ or other stress factors activate its enzyme activity as well as its expression. They also change its localization within the cell (Clarke et al., 2007; Ito et al., 2009). Deficiency of nSMase2 causes developmental retardation in bones and limbs (Stoffel et al., 2005; Aubin et al., 2005; Stoffel et al., 2007), despite its predominant expression in the CNS. The finding that nSMase2 plays a role in exosomes biogenesis in our system suggests a linkage between exosome release, stress response and development. However, the molecular and subcellular details of how nSMase2 regulates exosome biogenesis need further investigation.

One important question is also how the limiting membrane of an endosome is organized. In the case of the plasma membrane, the cytoplasmic leaflet contains less sphingomyelin as compared to the extracellular leaflet (Kiessling et al., 2009). It is likely that the same is true for the endosomal membrane. However, the small amounts of sphingomyelin in the inner leaflet together with the properties of ceramide to concentrate in domains, might be sufficient to promote vesicle budding into the lumen of an endosome. How sphingomyelin and ceramide are distributed in a membrane is another important question that need to be investigated in the future.

\subsubsection{Sorting of cargo into MVBs}

After endocytosis, proteins and lipids that are destined for lysosomal degradation are first incorporated into intraluminal vesicles of MVBs and are then delivered to lysosomes for degradation. How proteins and lipids are sorted to these subsets of ILVs and MVBs directed either for lysosomal degradation or for secretion as exosomes is unknown. To follow the sorting of an lysosomal and exosomal cargo, we studied the fate of the EGFR and PLP, respectively.

Using the GTP-locked of Rab5 ${ }^{\mathrm{Q} 79 \mathrm{~L}}$, we found that EGFR and PLP are both trapped in these enlarged endosomes and are separated into different domains. However, an important question is how PLP and EGFR behave in the absence of Rab5 ${ }^{\text {Q79L }}$. Are they sorted 
into distinct subpopulation of MVBs after entering early endosomes or do they separate at a later stage. The endosomal system contains a large number of different endosomal organelles, but so far it has not been possible to separate the endosomal populations that carry cargo to lysosomes from those that secrete their content as exosomes. Rab proteins show a characteristic subcellular distribution. They do indeed represent an important determinant of organelle identity and even characterize different domains one endosomes. For example, Rab5, Rab4, and Rab11 form distinct domains on early endosomes (Sönnichsen et al., 2000). We therefore performed a Rab screen in order to identify mechanisms in the intracellular transport of exosomal cargo, but also to define the secretory MVEs by Rab proteins.

The screen resulted in the identification of Rab35 as one essential Rab in the exosome pathway. Rab35 affected the release of exosomes in a GTP dependent manner (Figure 3.20). Rab35 has previously been identified as a regulator in a rapid recycling pathway in mammalian cells (Kouranti et al., 2006) and plays a role in a recycling route together with Rab11 (Sato et al., 2008). Our results are consistent with an earlier study, which proposed an involvement of the endosomal recycling pathway in exosome biogenesis. In this study Rab11 was shown to affect exosome release in K562 cells (Sönnichsen et al., 2000; Savina et al., 2002, 2005).

However, we did not succeed in our initial aim to identify a RabGTPase that marks a specific MVB subpopulatioNafor exosome release. We found that the majority of the GTP-bound form of Rab35 was present at the plasma membrane and only small amount of the GDP-bound form of Rab35 colocalized with PLP in endosomes (Figure 3.21). More work will be required to find suitable markers that allow a separation of MVB subpopulations. MVBs are dynamic structure that constantly changes their shape due to fusion and budding with vesicular carriers or other endosomal populations, but also as a consequence of endosomal maturation.

Back fusion of ILVs with the MVB limiting membrane is another way to create different MVB population. It has been proposed that a MVBs may contain different population of ILVs, of which some have the ability to back-fuse back the limiting membrane of the MVB. The back-fusion of ILVs is a similar type of process as the fusion of pathogens in particular viruses with the endosomal membrane (Gruenberg \& van der Goot, 2006; Fal- 
guières et al., 2009). Different back-fusion rate can change the molecular composition of the limiting membrane of a MVB. Possibly both the spatial separation of recycling endosomes and the regulation of balance between formation and back fusion of ILVs contribute to the diversity of MVBs.

To fully understand the how different cargoes are transported from early endosomes to different MVBs, further studies have to address the composition and organization of endosomal membrane, as well as characterization of different population of ILVs and MVBs.

\subsubsection{Existence of different exosome populations}

A few years after exosomes were discovered, it was suggested that different types of exosomes exist. For example, $50 \%$ of the exosomes secreted by reticulocytes lack the transferrin receptor (Johnstone et al., 1989). Also in our work, we have indirect evidence for different exosome populations secreted by Oli-neu cells.

We found Alix in Oli-neu exosomes. Alix is an ESCRT-associated protein, which binds to ESCRT-I and ESCRT-II. This can not be taken as a proof, but it may suggest that Alix enter the ILVs through a ESCRT-dependent manner. The exosomal secretion of other ESCRT components were not evaluated by Western blotting, however, with mass spectrometry two other ESCRT related proteins were identified (Table A.1): Tsg101 (ESCRT-I) and Nedd4 (Rsp5 in yeast), a E3 ubiquitin ligase, which mediates ubiquitylation of some protein cargoes targeted to MVBs (Katzmann et al., 2002). Together, this results provide indirect evidence for an involvement of the ESCRT machinery in a subpopulations of exosomes.

Alix is also known to play a role in the LBPA-dependent formation of ILVs in acidic endosomes. This process does not require ESCRT components. It is therefore also possible that Alix is involved in the generation of another type of ILVs - such that contain high amounts of LBPA. The LBPA containing ILVs are distinct from the ILVs that generated by the ESCRT machinery and that contain phosphatidylinositol-3-phosphate (PtdIns(3)P) (Gruenberg \& van der Goot, 2006). 
Due to the small size of ILVs being closely packed within MVBs it is technically challenging to identity subpopulations by immunoelectron microscopy. In addition, biochemical approaches to purify ILVs are even more demanding. One feasible possibility is to purify exosomes and use immunoelectron microscopy or FACS sorting of purified exosomes to distinguish the different populations. Such studies will be extremely important in order to characterize and classify the secreted vesicles in more detail.

Quantitative chemical assays on exosome composition together with the inhibition of different exosome/ILV biogenesis pathways may also give insight to this question.

\subsection{Rab35 and endosomal trafficking}

In order to identify the RabGTAases that are involved in exosome secretion pathway, we used two different approaches. A proteome analysis of Rab proteins in exosomes and a Rab GAP screen of exosome secretion. Using mass spectrometry we identified 11 different Rabs in the $100000 \mathrm{~g}$ pellet of Oli-neu cells: Rab1A, 1B, 2, 5B, 5C, 6A, 7, 8B, 10, 11B, and 35. Except of Rab8B, all the other Rab have previously been found in exosomes derived from other cell types (Table A.1). We expressed all of the identified Rab exogenously in Oli-neu cells and observed that Rab35 was the one that was secreted to the greatest extent. However, compared to PLP, all Rab proteins were secreted in a much smaller amount (Figure 3.17). How a Rab protein is released with exosomes is not clear and does not tell much about its functional involvement in the process. It is possible thtat a Rab protein is positioned in a membrane domain where intraluminal budding occurs, and is subsequently transported with the domain into the ILVs in a non specific fashion.

To functionally characterize the Rab proteins in exosomes secretion, we interfered with their function by screening a Rab GTPase-activating protein (GAP) library. These Rab GAPs were coexpressed with PLP in Oli-neu cells and the release of PLP into the extracellular medium was analyzed by biochemical methods. Candidate positives from this first round of screening were retested comparing the effects of the wild-type GAP to that of catalytically inactive point mutants. 
Using this method we identified a number of GAPs that increased exosome secretion. However, the increase in PLP recovery in the exosome pellet can also result from induction cell death by a particular Rab-GAPs leading to contamination of cell fragment in the pellet. Therefore, we were only interested in the Rab-GAPs having an inhibitory effect on exosome release and they were chosen for further study.. The 5 Rab-GAPs which reduced the PLP release with exosome to less than $60 \%$ were TBC1D10B, RN-tre, TBC1D10A, TBC1D10C and TBC1D15. Their inactive mutants did not have the inhibitory effect, showing the specificity of these Rab-GAPs in regulating exosome biogenesis (Figure 3.18).

It is not known, which Rab proteins are targeted by the different identified Rab-GAPs. Studies on RN-tre have given contradictory results. RN-tre was initially identified as a RabGAP for Rab5 (Lanzetti et al., 2000; Martinu et al., 2002). However, this has recently been challenged by providing evidence that $\mathrm{RN}$-tre targets Rab43 in a screen aimed at identifying Rabs involved in Shiga toxin transport from endosomes to the Golgi (Haas et al., 2007; Fuchs et al., 2007). TBC1D15 has been reported to target Rab7 GAP and therefore to regulate the function of late endosomes (Zhang et al., 2005).

TBC1D10A-C were also identified as regulators of Shiga toxin trafficking to the Golgi. The target of TBC1D10B was not identified with certainty in this study (Fuchs et al., 2007). One study showed that TBC1D10A (EPI64) is a Rab27a GAP (Itoh \& Fukuda, 2006) and TBC10D10C (EPI64C) was suggested to be a Rab35 GAP (Patino-Lopez et al., 2008). In this work, we reexamined this question and found that all members of the TBC1D10AC family increase the GTPase activity of Rab35. TBC1D10B was the strongest Rab35 GAP. The activity of towards Rab27a was small, if present at all (Figure 3.19). These results gave first evidence for a role of Rab35 in exosome release. Knocking down Rab35 with siRNA confirmed these results. More evidence came from experiments using mutant forms of Rab35, which showed that Rab35 regulates exosome secretion in a GTP dependent manner (Figure 3.20).

The molecular function of Rab35 is not fully understood. It has been suggested to be a regulator in the recycling endocytic pathway (Kouranti et al., 2006; Sato et al., 2008) and was found in peptide-MHC class II containing endosomes (Walseng et al., 2008). In our study, we observed as expected a GTP-dependent subcellular localization of Rab35 (Figure 3.21). The GTP-locked Rab35 ${ }^{\mathrm{Q} 67 \mathrm{~A}}$ was mainly found at the plasma membrane, where 
also TBC1D10B was located (Figures 3.21 and 3.22). The nucleotide empty Rab35 ${ }^{\text {N120I }}$ and the GDP-locked Rab35 ${ }^{\mathrm{S} 22 \mathrm{~N}}$ were mainly cytosolic, but small amounts were also present at endosomes that contained PLP and/or Lamp1. The subcellular localization of Rab35 was similar to previous studies, but the endosomal localization of Rab 35 was not as prominent in our cells as compared to previous reports (Kouranti et al., 2006; PatinoLopez et al., 2008). Theses studies also reported a change of endosome morphology after knockdown of Rab35 or expression of the dominant-negative form of Rab35. We only observed an enlargement of PLP containing endosomes after overexpression of Rab35 ${ }^{\mathrm{S} 22 \mathrm{~N}}$ or Rab35 ${ }^{\mathrm{N} 120 \mathrm{I}}$ after long expression times ( $48 \mathrm{hr}$, data not shown), but not after RNAi treatment of Rab35. Moreover, unlike Rab7, which plays important role in late endosomal transport, the expression of negative forms of Rab35 did not affect the co-localization of PLP and Lamp-1 or the degradation of EGF (Figures 3.21, 3.24 and 3.25). Together these pointed to function of Rab35 in process that is not related to protein degradation in lysosomes.

\subsection{MVB to plasma membrane trafficking}

There were a number of experimental findings that pointed to a role of Rab35 in the transport of MVBs to the plasma membrane. We found that knock-down of Rab35 with siRNA increased the number of PLP positive endosomes (Figure 3.26). This could be due to a trafficking jam of PLP containing endosomes to the plasma membrane, but possibly also to other cellular destinations. We examined the motility of these endosomes using live-imaging to get insights into a possible defect in plasma membrane targeting. We expressed the negative forms of Rab35 (Rab35 ${ }^{\mathrm{S} 22 \mathrm{~N}}$ and Rab35 ${ }^{\mathrm{N} 120 \mathrm{I}}$ ) and expected to find a reduced motility of LysoTracker lableled PLP-containing endosomes. However, we obtained the opposite result; it was the GTP-locked Rab35 ${ }^{\mathrm{Q} 67 \mathrm{~L}}$ which slowed the velocity of these endosomes (Figure 3.27). When TIRF experiments were performed to visualize the docked pool of endosomes at the plasma membrane, we observed that the GTP-locked Rab35 ${ }^{\mathrm{Q} 67 \mathrm{~L}}$ increased the fraction of endosomes directly beneath the plasma membrane. These endosomes were stationary and can therefore be considered as docked or tethered to the plasma membrane. Taking all these results together, it seems likely that Rab35 promotes the tethering of endosomes to the plasma membrane. This interpretation explains why the GTP-locked Rab35 ${ }^{\mathrm{Q} 67 \mathrm{~L}}$ slows down the movement of endosomal vesicles. 
It also explains why the pool of vesicle present at the plasma membrane is high when the GTP-locked Rab35 ${ }^{\mathrm{Q} 67 \mathrm{~L}}$ is expressed in a cell.

Recently other Rab proteins, Rab27a and Rab27b, were identified as regulators of the exosome secretion pathway in Hela cells (Ostrowski et al., 2009). In this paper, Rab27a and Rab27b were found to function in MVB docking at the plasma membrane. The size of MVBs increased by Rab27a silencing, whereas MVBs were redistributed towards the perinuclear region upon Rab27b silencing. Two Rab27 effectors were identified to mediate these effects, Slp4 (also known as SYTL4, synaptotagmin-like 4) and Slac2b (also known as EXPH5, exophilin 5).

Rab27a has previously been implicated in regulating melanosome movement by providing a connection to myosin Va through the adaptor protein, melanophilin. These Rab27a positive vesicles are transported to the cell periphery with myosin Va (Wu et al., 2002) and the distribution of melanosomes is controlled by synpaptotagmin-like protein 2 (SLP2/SYTL2) (Kuroda \& Fukuda, 2004).

How Rab35 regulates the tethering/docking of endosomes is not known. Our effector screen using a GST pull-down assay followed by mass spectrometry has so far not yielded any conclusive results. Recently, Rab35 was identified as a regulator of the actin cytoskeletion and this occurred by the effector protein, fascin (Zhang et al., 2009). In the mass spectrometry analysis of the same study, Munc 13-4 was also indentified as a putative Rab35 effector. Due to the well known function of Munc 13-4 in vesicle tethering/docking, we performed coimmunoprecipitation experiments to obtain evidence for a possible interaction of Munc 13-4 with Rab35. However, these experiments provided negative results suggesting that Munc 13-4 is not a Rab35 effector.

As depicted in Figure 1.4, to fuse with the target membrane, a vesicle approaching the target membrane, has to be recruited by tethers to facilitate the assembly of SNARE complexes to allow efficient fusion. It will also be important to identify the responsible SNARE proteins required for exosome secretion. 


\subsection{Function of exosomes}

As introduced in Section 1.1.2, there a number of possible function for exosomes. The function of exosomes secreted by oligodendrocytes has not been studied. Because oligodendrocytes are the myelinating cells of the CNS, it is possible that regulate the signaling between neuron and glia or myelination. Another perhaps more likely function of exosomes from oligodendrocytes, is waste disposal. Oligodendrocytes produce tremendous amounts of membrane and it is possible that release of excess membrane by exosomes is an efficient was of removing obsolete proteins or lipids. The exosomes within the extracellular space are possibly taken up by cells, which are specialized for clearance, such as microglia or macrophages.

The lipid composition of exosomes may not only be a key to understand their biogenesis, but also point to their possible function. It has been shown that the $\mathrm{T}$ cell derived exosomes contain phosphatidylserine (PS) in the outer membrane leaflet and can induce the pro-inflammatory reaction of monocytes through PS receptor (Zakharova et al., 2007). Endocytosis of carcinoma exosomes into nature killer cells is also PS dependent (Keller et al., 2009). The exposed PS of apoptotic cells and cell debris is a signal for phagocytic cells for removal of these fragments. It is possible that exosomes secreted by oligodendrocytes are taken up in a similar fashion by microglia, the macrophage-like cells in the CNS having the ability to remove apoptotic neurons (Witting et al., 2000). This possible phagotic clearance of exosomes might be a way for oligodendrocytes to remove excess proteins or lipids, in a similar as in reticulocytes.

The finding that MVBs are present in the cytoplasmic channels within the abaxonal space of myelin, suggests that endosomal trafficking still occurs in the non-compact region of myelin. Together with the fact that Rab35, which regulates exosome secretion, was isolated from non-compact myelin, these data suggest that exosomes secretion may occur at these sites. If exosomes are produced by oligodendrocytes within the abaxonal space of myelin, they may be shuttle vectors for cargoes or signal molecules to axons. One interesting study showed that Schwann cells can transfer functional ribosomes to axons, supporting the survival of desomatized (cell body removed) axons (Court et al., 2008). Intra-cellular organelle transport also occurs in other cell types, such as the transfer of melanosomes, a lysosome related organelle, from melanocytes to keratinocytes (Seiberg, 
2001). Furthermore, exosomes that contain mRNA and microRNA (Valadi et al., 2007) are transported from one cell to another.

Because exosomes are present in body fluids, it is possible that exosomes from oligodendrocytes can be transfered to cerebrospinal fluid or even to the peripheral blood circulations. This might be relevant in respect to the possible role of exosomes in transferring antigens to antigen presenting cells in immune competent organs (Section 1.1.2).

If this indeed occurs, the myelin antigens present in exosomes might stimulate the immune system and lead to autoimmune diseases such as multiple sclerosis. Immune response caused by myelin antigens, including PLP, can cause experimental autoimmune encephalomyelitis (EAE), which is an animal model for multiple sclerosis, an inflammatory demyelinating disease (Gold et al., 2000). Therefore, it will be interesting to study whether PLP and/or other myelin antigens transported by exosomes can induce immune response and whether the leakage of oligodendroglial exosomes into the peripheral circulation can be one cause of multiple sclerosis. 


\section{Chapter 5}

\section{Summary and conclusion}

Using an oligodendroglial cell line that contains large quantities of multivesicular endosomes, we have addressed the question of how exosomes are formed. Exosomes are vesicles of endocytic origin that are released into the extracellular environment after fusion of multivesicular endosomes with the plasma membrane.

We found that the exosomal cargo segregates together into distinct microdomains on the endosomal membrane. The transfer of these microdomains into the lumen of the endosome did not depend on the function of the ESCRT-machinery, but required ceramide. The lipid analysis of exosomes revealed an enrichment in ceramide and the formation of exosomes was sensitive to the inhibition of neutral sphingomyelinases, but insensitive to the functional inhibition of components of the ESCRT-complex. Using giant unilamellar liposomes we show that the addition of sphingomyelinase was sufficient to induce the inward-budding of lipid rafts into a liposome. Taken together, our results establish a novel inward-budding pathway into a vesicle, by the generation of ceramide from sphingomyelin. We propose that these microdomains act as collecting devices for the lateral segregation of cargo in the limiting membrane of endosomes and that the formation of ceramide from sphingomyelin within these microdomains triggers the budding process into the multivesicular endosome. Ceramide might be a driving force to generate a vesicle as it has a cone-shaped structure, which might induce negative spontaneous curvature by creating an area difference between the membrane bilayer.

These results represent a novel mechanism of how a vesicle is formed from a membrane. 
In the second part of the thesis we studied the machinery involved in exosome biogenesis.

Since the Rab family guanosine triphosphatases (GTPases) proteins regulate intracellular membrane trafficking, we studied the role of Rab proteins in exosome release in oliogodendroglial cells.

To define the Rab proteins required in exosome biogenesis, a Rab GTPase-activating protein (GAP) library was screened for the ability of each Rab GAP to reduce exosome secretion.

This screen resulted in several major novel insights:

1. The TBC1D10 family of Rab GAPs regulate exosome secretion in a catalytic activitydependent manner.

2. Rab35 is the target of TBC1D10A-C

3. Inhibition of Rab35 function impairs exosome biogenesis.

4. Rab35 regulates exosome generation by controlling the targeting of endocytic vesicles to the plasma membrane.

Together, this work brings new insight into the molecular mechanisms of exosome biogenesis. 


\section{Appendix A}

\section{Oli-neu exosomal proteins}

Table A.1: Proteins in Oli-neu derived exosomes. Proteins from exosomes collected in the culture medium wild-type Oli-neu cells were analysized with chromatography coupled tandom mass spectrometry. A totoal amount of 301 proteins were found in the exosomes and were compared to a selection of previously reported exosomal proteins originated from other cells. 103 proteins were found in exosomes secreted by the following cells/tissue: a, adipocytes (Aoki et al., 2007); b, kidneys (Barile et al., 2005); c, hepatocytes (Conde-Vancells et al., 2008); s, dendritic cells (Segura et al., 2005); v, mast cells (Valadi et al., 2007).

\begin{tabular}{|c|c|c|c|}
\hline No. & GI number & Protein Name & $\begin{array}{c}\text { Found in } \\
\text { other cells }\end{array}$ \\
\hline 1 & gi $\mid 27754140$ & 1110059P08Rik protein & \\
\hline 2 & gi| 1526539 & $14-3-3$ zeta & $\mathrm{abcs} v$ \\
\hline 3 & gi|2160434 & 2,3 -cyclic-nucleotide 3 -phosphodiesterase I & \\
\hline 4 & gi| 2253401 & $23 \mathrm{kDa}$ synaptosomal associated protein & \\
\hline 5 & gi|112804 & 4F2 cell-surface antigen heavy chain (4F2hc) & $\mathrm{c} \mathrm{v}$ \\
\hline 6 & gi|6754900 & 5 nucleotidase, ecto & \\
\hline 7 & gi|6606074 & $60 \mathrm{kDa}$ ribonucleoprotein $\mathrm{SSA} / \mathrm{Ro}$ & \\
\hline 8 & gi| 899445 & $60 \mathrm{~S}$ ribosomal protein & \\
\hline 9 & gi| 2500367 & $60 S$ ribosomal protein L21 & $\mathrm{v}$ \\
\hline 10 & gi|6671569 & acidic ribosomal phosphoprotein $\mathrm{P} 0$ & $\mathrm{~b} \mathrm{v}$ \\
\hline 11 & gi| 29839749 & $\begin{array}{l}\text { ADAM } 10 \text { precursor (A disintegrin and metalloproteinase domain } \\
\text { 10) (Mammalian disintegrin-metalloprotease) (Kuzbanian protein ho- } \\
\text { molog) (CD156c antigen) }\end{array}$ & \\
\hline 12 & gi|4502201 & ADP-ribosylation factor 1 & $\mathrm{~b} \mathrm{c} \mathrm{v}$ \\
\hline 13 & gi|6671571 & ADP-ribosylation factor 2 & $\mathrm{~b}$ \\
\hline 14 & gi|6671539 & aldolase $1, \mathrm{~A}$ isoform & \\
\hline 15 & gi|786001 & aldose reductase & \\
\hline 16 & gi|4633515 & ALG-2 interacting protein AIP1 & $\mathrm{cs}$ \\
\hline 17 & gi| 27966961 & alpha-2-macroglobulin-P & $\mathrm{a}$ \\
\hline 18 & gi $\mid 431416$ & alpha-7 integrin & \\
\hline 19 & gi|387090 & alpha-cardiac actin & $\mathrm{c} \mathrm{v}$ \\
\hline 20 & gi $\mid 202210$ & alpha-tubulin isotype M-alpha-2 & \\
\hline 21 & gi|49942 & AM2 receptor & \\
\hline 22 & gi|17225630 & AN2/NG2 proteoglycan & \\
\hline 23 & gi|6996913 & annexin A2 & $\mathrm{abcs}$ \\
\hline 24 & gi|6753060 & annexin A5 & b c s v \\
\hline 25 & gi $\mid 1870400$ & anti-DNA immunoglobulin light chain IgG & \\
\hline
\end{tabular}




\begin{tabular}{|c|c|c|c|}
\hline 26 & gi|2745924 & anti-PC Ig kappa chain & \\
\hline 27 & gi|70912321 & Anxa1 & \\
\hline 28 & gi|16923986 & aplysia ras-related homolog A2 & \\
\hline 29 & gi $\mid 6680710$ & aquaporin 1 & $\mathrm{~b}$ \\
\hline 30 & gi $\mid 871422$ & aspartate aminotransferase & $\mathrm{s}$ \\
\hline 31 & gi|148677499 & $\begin{array}{l}\text { ATP synthase, H+ transporting, mitochondrial F1 complex, alpha } \\
\text { subunit, isoform 1, isoform CRA_c }\end{array}$ & \\
\hline 32 & gi|148677501 & $\begin{array}{l}\text { ATP synthase, } \mathrm{H}+\text { transporting, mitochondrial F1 complex, alpha } \\
\text { subunit, isoform 1, isoform CRA_e }\end{array}$ & \\
\hline 33 & gi|6752944 & ATP-binding cassette, sub-family G, member 2 & \\
\hline 34 & gi|16307541 & Atp1a1 protein & b c \\
\hline 35 & gi|23272966 & Atp5b protein & $\mathrm{b}$ \\
\hline 36 & gi|6978545 & ATPase, $\mathrm{Na}+/ \mathrm{K}+$ transporting, alpha 2 polypeptide & $\mathrm{b}$ \\
\hline 37 & gi $\mid 2808470$ & basigin & \\
\hline 38 & gi $\mid 45598372$ & brain abundant, membrane attached signal protein 1 & \\
\hline 39 & gi|90342 & calcium-binding protein (clone pMP41) - mouse (fragment) & \\
\hline 40 & gi|6671672 & capping protein (actin filament) muscle Z-line, alpha 2 & $\mathrm{v}$ \\
\hline 41 & gi|14331139 & carboxylesterase ML1 & \\
\hline 42 & gi|6671684 & catenin (cadherin associated protein), beta $1,88 \mathrm{kDa}$ & $\mathrm{bs}$ \\
\hline 43 & gi|19526794 & CD 81 antigen & \\
\hline 44 & gi|53674 & CD44 & \\
\hline 45 & gi|6754382 & CD47 antigen & \\
\hline 46 & gi 6680888 & Cd63 antigen & $\mathrm{av}$ \\
\hline 47 & gi|6680894 & CD9 antigen & as \\
\hline 48 & gi|4757952 & cell division cycle 42 isoform 1 & \\
\hline 49 & gi|38372905 & cell line NK14 derived transforming oncogene & \\
\hline 50 & gi| 2735712 & cell surface molecule OX-2 & \\
\hline 51 & gi|576133 & $\begin{array}{l}\text { Chain A, 1.8 Angstroms Molecular Structure Of Mouse Liver Class Pi } \\
\text { Glutathione S-Transferase Complexed With S-(P-Nitrobenzyl) Glu- } \\
\text { tathione And Other Inhibitors }\end{array}$ & \\
\hline 52 & gi|5542285 & $\begin{array}{l}\text { Chain A, Crystal Structure Of Macrophage Migration Inhibitory Fac- } \\
\text { tor Complexed With (E)-2-Fluoro-P-Hydroxycinnamate }\end{array}$ & \\
\hline 53 & gi|62738726 & Chain A, VcpP97 COMPLEXED WITH ADP & \\
\hline 54 & gi|6671702 & chaperonin subunit 5 (epsilon) & \\
\hline 55 & gi|146231960 & chondroitin sulfate proteoglycan 4 & \\
\hline 56 & gi|6680924 & cofilin 1, non-muscle & $\mathrm{c} \mathrm{s} \mathrm{v}$ \\
\hline 57 & gi|6671746 & cofilin 2 , muscle & \\
\hline 58 & gi| 2493781 & Collagen alpha-1(XI) chain precursor & \\
\hline 59 & gi|148706297 & complement component 3, isoform CRA_d & a s \\
\hline 60 & gi|14193682 & copine 1 protein & $\mathrm{s}$ \\
\hline 61 & gi|25141335 & copine III & $\mathrm{v}$ \\
\hline 62 & gi|21630253 & copine VIII isoform 1 & \\
\hline 63 & gi|19483894 & Cpne2 protein & \\
\hline 64 & gi|10946574 & creatine kinase, brain & \\
\hline 65 & gi|6753530 & crystallin, alpha B & $\mathrm{b}$ \\
\hline 66 & gi|9790219 & destrin & \\
\hline 67 & gi|6681137 & diazepam binding inhibitor isoform 2 & \\
\hline 68 & gi $\mid 6680297$ & DnaJ (Hsp40) homolog, subfamily A, member 1 & \\
\hline 69 & gi|9789937 & DnaJ (Hsp40) homolog, subfamily A, member 2 & \\
\hline 70 & gi|22256949 & $\begin{array}{l}\text { Dolichyl-diphosphooligosaccharide-protein glycosyltransferase } 48 \\
\mathrm{kDa} \text { subunit precursor (Oligosaccharyl transferase } 48 \mathrm{kDa} \text { subunit) } \\
\text { (DDOST } 48 \mathrm{kDa} \text { subunit) }\end{array}$ & \\
\hline 71 & gi|14017413 & dudulin 2 & \\
\hline
\end{tabular}




\begin{tabular}{|c|c|c|c|}
\hline 72 & gi|1155351 & dystroglycan & \\
\hline 73 & gi|46879190 & EGF-like repeats and discoidin I-like domains 3 isoform b & \\
\hline 74 & gi|10181214 & EH-domain containing 3 & \\
\hline 75 & gi|31981592 & EH-domain containing 4 & $\mathrm{v}$ \\
\hline 76 & gi|556301 & elongation factor $\mathrm{Tu}$ & \\
\hline 77 & gi|309215 & EndoA cytokeratin (5 end put.); putative & \\
\hline 78 & gi|1196645 & $\begin{array}{l}\text { env gene ORF starts at base } 65 \text {; first start codon is found at } 218 \text {; } \\
\text { putative }\end{array}$ & \\
\hline 79 & gi|387397 & epidermal keratin subunit I & \\
\hline 80 & gi| 293686 & epidermal keratin subunit II & \\
\hline 81 & gi|9506571 & eukaryotic translation initiation factor 2 , subunit 1 alpha & \\
\hline 82 & gi|110349752 & FERMRhoGEF (Arhgef) and pleckstrin domain protein 1 & \\
\hline 83 & gi|46849812 & fibronectin 1 & $\mathrm{ab}$ \\
\hline 84 & gi|6679809 & flotillin 1 & $\mathrm{~s}$ \\
\hline 85 & gi|29126933 & Fmnl2 protein & \\
\hline 86 & gi $\mid 4262551$ & G-protein XLalphas & \\
\hline 87 & gi|86651881 & gag polyprotein pr65 & \\
\hline 88 & gi|1498648 & Gag-Pol polyprotein & \\
\hline 89 & gi|86651882 & gag-pro-pol polyprotein & \\
\hline 90 & gi|86651866 & gag-pro-pol polyprotein & \\
\hline 91 & gi|809561 & gamma-actin & $\mathrm{b} \mathrm{s} \mathrm{v}$ \\
\hline 92 & gi|32171368 & Gap junction epsilon-1 protein (Connexin-29) (Cx29) & \\
\hline 93 & gi|76253900 & GDP dissociation inhibitor 2 [Bos taurus] & \\
\hline 94 & gi|6680067 & glucose phosphate isomerase 1 & $\mathrm{v}$ \\
\hline 95 & gi|6679937 & glyceraldehyde-3-phosphate dehydrogenase & $\mathrm{as} \mathrm{v}$ \\
\hline 96 & gi| 27805887 & $\begin{array}{l}\text { guanine nucleotide binding protein ( } \mathrm{G} \text { protein), alpha inhibiting ac- } \\
\text { tivity polypeptide } 1 \text { [Bos taurus] }\end{array}$ & \\
\hline 97 & gi|13384618 & guanine nucleotide binding protein ( $\mathrm{G}$ protein), gamma 12 & \\
\hline 98 & gi|33563256 & guanine nucleotide binding protein, alpha inhibiting 3 & $\mathrm{~b} \mathrm{~s}$ \\
\hline 99 & gi|6680043 & guanine nucleotide binding protein, alpha transducing 2 & \\
\hline 100 & Gi|1730229 & $\begin{array}{l}\text { Guanine nucleotide-binding protein } \mathrm{G}(\mathrm{i}) \text {, alpha-2 subunit (Adenylate } \\
\text { cyclase-inhibiting } \mathrm{G} \text { alpha protein) }\end{array}$ & $\mathrm{c} \mathrm{v}$ \\
\hline 101 & gi|120979 & Guanine nucleotide-binding protein $\mathrm{G}(\mathrm{o})$ subunit alpha 2 & \\
\hline 102 & gi|6680045 & guanine nucleotide-binding protein, beta- 1 subunit & $\mathrm{b} \mathrm{v}$ \\
\hline 103 & Gi|29244126 & H2A histone family, member $\mathrm{J}$ & $\mathrm{c}$ \\
\hline 104 & gi|7106331 & H2A histone family, member $\mathrm{X}$ & $\mathrm{v}$ \\
\hline 105 & gi|41152517 & H2A histone family, member Y & $\mathrm{c}$ \\
\hline 106 & gi $\mid 4504255$ & H2A histone family, member $\mathrm{Z}$ & $\mathrm{b} \mathrm{v}$ \\
\hline 107 & gi|30061401 & H3 histone, family 2 isoform 2 & \\
\hline 108 & gi|4504301 & H4 histone family, member A & $\mathrm{c} \mathrm{v}$ \\
\hline 109 & gi|2495342 & Heat shock 70 kDa protein 4 (Heat shock 70-related protein APG-2) & \\
\hline 110 & gi|6754254 & heat shock protein 1 , alpha & \\
\hline 111 & Gi|31981722 & heat shock protein 5 & $\mathrm{~b}$ \\
\hline 112 & gi|309319 & heat shock protein 70 cognate & $\mathrm{b} \mathrm{c}$ \\
\hline 113 & gi|1706186 & $\begin{array}{l}\text { High affinity cationic amino acid transporter } 1 \text { (CAT-1) (CAT1) (Sys- } \\
\text { tem Y+ basic amino acid transporter) (Ecotropic retroviral leukemia } \\
\text { receptor) (ERR) (Ecotropic retrovirus receptor) }\end{array}$ & \\
\hline 114 & gi|33468857 & histidine triad nucleotide binding protein 1 & \\
\hline 115 & gi|10800130 & histone 1, H2ad & $\mathrm{c} \mathrm{v}$ \\
\hline 116 & gi| 28316750 & histone 1, H2ba & $\mathrm{c}$ \\
\hline 117 & gi|30089704 & histone $1, \mathrm{H} 2 \mathrm{bk}$ & $\mathrm{c}$ \\
\hline 118 & gi|1170155 & Histone H1.3 (H1 VAR.4) (H1d) & $\mathrm{abv}$ \\
\hline 119 & gi|554126 & IgK chain & \\
\hline
\end{tabular}




\begin{tabular}{|c|c|c|c|}
\hline 120 & gi| 55346 & immunoglobulin light chain & \\
\hline 121 & gi|15593237 & immunoglobulin superfamily receptor PGRL & $\mathrm{s}$ \\
\hline 122 & gi|31982236 & integrin alpha 6 & \\
\hline 123 & gi $\mid 47717127$ & integrin alpha 8 & \\
\hline 124 & gi|46402185 & integrin alpha FG-GAP repeat containing 3 & \\
\hline 125 & gi|123781093 & $\begin{array}{l}\text { Integrin alpha-1 precursor (Laminin and collagen receptor) (VLA-1) } \\
\text { (CD49a antigen) }\end{array}$ & \\
\hline 126 & gi|1170592 & $\begin{array}{l}\text { Integrin alpha- } \mathrm{V} \text { precursor (Vitronectin receptor subunit alpha) } \\
\text { (CD51 antigen) [Contains: Integrin alpha-V heavy chain; Integrin } \\
\text { alpha-V light chain] }\end{array}$ & \\
\hline 127 & gi|7949057 & integrin beta 3 & \\
\hline 128 & gi|21489935 & keratin 14 & \\
\hline 129 & gi|741022 & keratin 15 & $\mathrm{v}$ \\
\hline 130 & gi|38503465 & keratin $17 \mathrm{n}$ & \\
\hline 131 & gi|111308159 & Keratin 2 & \\
\hline 132 & gi|54607171 & keratin $6 \mathrm{~A}$ & $\mathrm{v}$ \\
\hline 133 & gi|22164776 & keratin $6 \mathrm{~L}$ & \\
\hline 134 & gi|14861854 & keratin 7 & \\
\hline 135 & gi|47059013 & keratin 73 & \\
\hline 136 & gi|51092293 & keratin 77 & \\
\hline 137 & gi|293682 & keratin D & \\
\hline 138 & gi|145207282 & LOC72520 protein & \\
\hline 139 & gi|547841 & Low-density lipoprotein receptor precursor (LDL receptor) & \\
\hline 140 & gi|4741831 & LR8 & \\
\hline 141 & gi|126381 & $\begin{array}{l}\text { Lysosome-associated membrane glycoprotein } 2 \text { precursor (LAMP-2) } \\
\text { (Lysosomal membrane glycoprotein type B) (LGP-B) (CD107b anti- } \\
\text { gen) }\end{array}$ & \\
\hline 142 & gi|695638 & M-TAXREB107 & \\
\hline 143 & gi|13879460 & Major vault protein & $\mathrm{ac}$ \\
\hline 144 & gi|6754706 & MARCKS-like 1 & \\
\hline 145 & gi|495128 & $\mathrm{mCBP}$ & \\
\hline 146 & gi|148695947 & mCG1040062 & \\
\hline 147 & gi|148668656 & mCG113987 & \\
\hline 148 & gi|148676955 & mCG118787 & \\
\hline 149 & gi|148664912 & mCG126569 & \\
\hline 150 & gi|148665111 & mCG126588, isoform CRA_a & \\
\hline 151 & gi|148707761 & mCG131000, isoform CRA_a & \\
\hline 152 & gi|88682939 & Melanoma antigen & \\
\hline 153 & gi|12746444 & melanoma cell adhesion molecule & \\
\hline 154 & gi|2143496 & membrane glycoprotein M6 - mouse & \\
\hline 155 & gi|4586464 & milk fat globule glycoprotein MFG-E8 S & a c \\
\hline 156 & gi|113865977 & milk fat globule-EGF factor 8 protein isoform 2 & $\mathrm{c}$ \\
\hline 157 & gi|6970476 & Misshapen/NIKs-related kinase MINK-1 & \\
\hline 158 & gi|145279237 & mitogen-activated protein kinase kinase kinase kinase 4 & \\
\hline 159 & gi|33438248 & mKIAA0034 protein & \\
\hline 160 & gi|50510423 & mKIAA0248 protein & \\
\hline 161 & gi|37359868 & mKIAA0253 protein & \\
\hline 162 & gi|50510473 & mKIAA0400 protein & \\
\hline 163 & gi|50510847 & mKIAA1207 protein & \\
\hline 164 & gi|28972760 & mKIAA1466 protein & \\
\hline 165 & gi|37360610 & mKIAA2014 protein & \\
\hline 166 & gi|70778915 & moesin & $\mathrm{c} \mathrm{v}$ \\
\hline 167 & gi|6678920 & Moloney leukemia virus 10 & \\
\hline
\end{tabular}




\begin{tabular}{|c|c|c|c|}
\hline 168 & gi|3328089 & monoclonal anti-DNA IgM kappa-chain variable region & \\
\hline 169 & gi|6688786 & mouse fat 1 cadherin & \\
\hline 170 & gi|148271067 & myeloid-associated differentiation marker & \\
\hline 171 & gi|148699227 & myeloid-associated differentiation marker, isoform CRA_b & \\
\hline 172 & gi|6678986 & myosin IC isoform b & \\
\hline 173 & gi|1924961 & myosin-I beta & \\
\hline 174 & gi|6678768 & myristoylated alanine rich protein kinase $\mathrm{C}$ substrate & \\
\hline 175 & gi|7242138 & $\mathrm{Na}+/ \mathrm{K}+-$ ATPase beta 2 subunit & \\
\hline 176 & gi|6680744 & $\mathrm{Na}+/ \mathrm{K}+-$ ATPase beta 3 subunit & \\
\hline 177 & gi|51895883 & Nedd4l protein & \\
\hline 178 & gi|148693781 & neural cell adhesion molecule 1, isoform CRA_b & \\
\hline 179 & gi|6679134 & neuropilin 1 & $\mathrm{~s}$ \\
\hline 180 & gi|148694008 & neuroplastin, isoform CRA_a & \\
\hline 181 & gi|3024586 & $\begin{array}{l}\text { Neutral amino acid transporter A (SATT) (Solute carrier fam- } \\
\text { ily } 1 \text { member 4) (Alanine/serine/cysteine/ threonine transporter) } \\
\text { (ASCT1) }\end{array}$ & \\
\hline 182 & gi|13529464 & Nucleolin & $\mathrm{v}$ \\
\hline 183 & gi|6679078 & nucleoside-diphosphate kinase 2 & \\
\hline 184 & gi|7657417 & odd Oz/ten-m homolog 3 & \\
\hline 185 & gi|200179 & P2B/LAMP-1 & $\mathrm{c}$ \\
\hline 186 & gi $\mid 6225814$ & $\begin{array}{l}\text { P2X purinoceptor } 7 \text { (ATP receptor) (P2X7) (Purinergic receptor) } \\
\text { (P2Z receptor) }\end{array}$ & \\
\hline 187 & gi|6679439 & peptidylprolyl isomerase $\mathrm{A}$ & $\mathrm{s} \mathrm{v}$ \\
\hline 188 & gi|122891114 & perlecan (heparan sulfate proteoglycan 2) & $\mathrm{a}$ \\
\hline 189 & gi|6754976 & peroxiredoxin 1 & $\mathrm{cs}$ \\
\hline 190 & gi|9506705 & phospholemman precursor & \\
\hline 191 & gi|200397 & phospholipase C-alpha & \\
\hline 192 & gi|54292132 & phosphoserine aminotransferase 1 & \\
\hline 193 & gi $\mid 62234487$ & plasma membrane calcium ATPase 1 & \\
\hline 194 & gi| 54261793 & plasma membrane calcium ATPase 4 & \\
\hline 195 & gi|6754994 & poly $(\mathrm{rC})$ binding protein 1 & $\mathrm{~b}$ \\
\hline 196 & gi|129535 & $\begin{array}{l}\text { Polyadenylate-binding protein } 1 \text { (Poly(A)-binding protein 1) (PABP } \\
\text { 1) }\end{array}$ & \\
\hline 197 & gi|7305201 & $\begin{array}{l}\text { potassium voltage-gated channel, shaker-related, subfamily, member } \\
6\end{array}$ & \\
\hline 198 & gi| 50845420 & prostaglandin F2 receptor negative regulator precursor & \\
\hline 199 & gi|6755891 & protease, serine, 3 & \\
\hline 200 & gi|6755198 & proteasome (prosome, macropain) subunit, alpha type 6 & \\
\hline 201 & gi|6755202 & proteasome beta 3 subunit & $\mathrm{v}$ \\
\hline 202 & gi| 2118156 & proteasome endopeptidase complex (EC 3.4.25.1) delta chain - mouse & \\
\hline 203 & gi| 226005 & protein $40 \mathrm{kD}$ & \\
\hline 204 & gi| 58037267 & protein disulfide isomerase-associated 6 & \\
\hline 205 & gi|7106381 & protein kinase $\mathrm{C}$ and casein kinase substrate in neurons 2 & \\
\hline 206 & gi|6679557 & protein tyrosine phosphatase, receptor type, A & \\
\hline 207 & gi|200409 & proteolipid protein variant $\mathrm{Dm}-20$ & \\
\hline 208 & gi|2499903 & Puromycin-sensitive aminopeptidase (PSA) & \\
\hline 209 & gi|7710086 & RAB10, member RAS oncogene family & $\mathrm{b} \mathrm{s}$ \\
\hline 210 & gi|6679583 & RAB11B, member RAS oncogene family & b s \\
\hline 211 & gi|4758988 & RAB1A, member RAS oncogene family & $\mathrm{b} \mathrm{c}$ \\
\hline 212 & gi|21313162 & RAB1B, member RAS oncogene family & $\mathrm{c}$ \\
\hline 213 & gi|10946940 & RAB2, member RAS oncogene family & $\mathrm{bcs}$ \\
\hline 214 & gi|5803135 & RAB35, member RAS oncogene family & $\mathrm{c}$ \\
\hline 215 & gi| 4506371 & RAB5B, member RAS oncogene family & b s \\
\hline
\end{tabular}




\begin{tabular}{|c|c|c|c|}
\hline 216 & gi|20072723 & Rab5c protein & $\mathrm{b} \mathrm{v}$ \\
\hline 217 & gi|13195674 & RAB6A, member RAS oncogene family & $\mathrm{s}$ \\
\hline 218 & gi|1050551 & rab7 & $\mathrm{bcs}$ \\
\hline 219 & gi| 23463313 & RAB8B, member RAS oncogene family & \\
\hline 220 & gi|4506413 & RAP1A, member of RAS oncogene family & $\mathrm{b} \mathrm{v}$ \\
\hline 221 & gi|7661678 & RAP1B, member of RAS oncogene family & $\mathrm{b}$ \\
\hline 222 & gi|10119859 & Ras homolog & \\
\hline 223 & gi|4757764 & ras homolog gene family, member B & \\
\hline 224 & gi|6680728 & ras homolog gene family, member $\mathrm{C}$ & \\
\hline 225 & gi|148687100 & RAS-related C3 botulinum substrate 1, isoform CRA_b & $\mathrm{v}$ \\
\hline 226 & gi|13399308 & related RAS viral (r-ras) oncogene homolog 2 & \\
\hline 227 & gi| 27735176 & Retrovirus-related Env polyprotein from $\mathrm{Fv}-4$ locus & $\mathrm{v}$ \\
\hline 228 & gi|13435747 & Rho GDP dissociation inhibitor (GDI) alpha & \\
\hline 229 & gi|198578 & ribosomal protein & \\
\hline 230 & gi| 200770 & ribosomal protein & \\
\hline 231 & gi|13592009 & ribosomal protein L10a & \\
\hline 232 & gi|13385408 & ribosomal protein L11 & $\mathrm{b} \mathrm{c}$ \\
\hline 233 & gi|31981945 & ribosomal protein L13a & $\mathrm{b} \mathrm{v}$ \\
\hline 234 & gi|13385472 & ribosomal protein L14 & $\mathrm{v}$ \\
\hline 235 & gi| 47059006 & ribosomal protein L18a & a s v \\
\hline 236 & gi|4506609 & ribosomal protein L19 & \\
\hline 237 & gi|4506619 & ribosomal protein L24 & $\mathrm{v}$ \\
\hline 238 & gi|4506631 & ribosomal protein L30 & $\mathrm{b} \mathrm{v}$ \\
\hline 239 & gi $\mid 4506633$ & ribosomal protein L31 isoform 1 & $\mathrm{~b}$ \\
\hline 240 & gi|13385044 & ribosomal protein L35 & \\
\hline 241 & gi|16117794 & ribosomal protein L36 & \\
\hline 242 & gi|9055322 & ribosomal protein L36 & $\mathrm{v}$ \\
\hline 243 & gi|4506663 & ribosomal protein L8 & $\mathrm{b} \mathrm{v}$ \\
\hline 244 & gi|4506681 & ribosomal protein S11 & $\mathrm{b} \mathrm{v}$ \\
\hline 245 & gi|4506685 & ribosomal protein S13 & $\mathrm{b} \mathrm{v}$ \\
\hline 246 & gi|3097244 & ribosomal protein S14 & $\mathrm{v}$ \\
\hline 247 & gi|4506691 & ribosomal protein S16 & $\mathrm{b} \mathrm{v}$ \\
\hline 248 & gi|12963511 & ribosomal protein S19 & $\mathrm{v}$ \\
\hline 249 & gi|4506703 & ribosomal protein S24 isoform c & $\mathrm{v}$ \\
\hline 250 & gi|6981488 & ribosomal protein S26 & $\mathrm{b} \mathrm{v}$ \\
\hline 251 & gi $\mid 6755372$ & ribosomal protein S3 & $\mathrm{bc} \mathrm{v}$ \\
\hline 252 & gi|4506743 & ribosomal protein $\mathrm{S} 8$ & $\mathrm{~b} \mathrm{v}$ \\
\hline 253 & gi| 83745120 & ribosomal protein, large $\mathrm{P} 2$ & $\mathrm{~b}$ \\
\hline 254 & gi|9790083 & RuvB-like protein 1 & \\
\hline 255 & gi|6755382 & RuvB-like protein 2 & \\
\hline 256 & gi|6006811 & serpentine receptor & \\
\hline 257 & gi|5915682 & Serum albumin precursor & $\mathrm{c} \mathrm{v}$ \\
\hline 258 & gi|7305485 & SH3-domain GRB2-like 1 & \\
\hline 259 & gi|6755662 & signal recognition particle 9 & \\
\hline 260 & gi| 104294890 & signaling molecule ATTP & \\
\hline 261 & gi|19353540 & Slc6a8 protein & \\
\hline 262 & gi|19855078 & $\begin{array}{l}\text { Sodium/potassium-transporting ATPase subunit alpha-3 (Sodium } \\
\text { pump subunit alpha-3) }(\mathrm{Na}(+) / \mathrm{K}(+) \text { ATPase alpha-3 subunit) } \\
(\mathrm{Na}(+) / \mathrm{K}(+) \text { ATPase alpha(III) subunit) } \\
\end{array}$ & \\
\hline 263 & gi|6677995 & solute carrier family 16, member 1 & \\
\hline 264 & gi|17376564 & $\begin{array}{l}\text { Solute carrier family } 2 \text {, facilitated glucose transporter member } 1 \text { (Glu- } \\
\text { cose transporter type 1, erythrocyte/brain) (GLUT-1) (GT1) }\end{array}$ & $\mathrm{c}$ \\
\hline 265 & gi|6681233 & solute carrier family 26 (sulfate transporter), member 2 & \\
\hline
\end{tabular}




\begin{tabular}{|c|c|c|c|}
\hline 266 & gi|109730173 & Solute carrier family 44, member 1 & \\
\hline 267 & gi|31982764 & $\begin{array}{l}\text { solute carrier family } 7 \text { (cationic amino acid transporter, y+ system), } \\
\text { member } 5\end{array}$ & \\
\hline 268 & gi|6755566 & $\begin{array}{l}\text { solute carrier family } 9 \text { (sodium/hydrogen exchanger), isoform } 3 \text { regu- } \\
\text { lator } 1\end{array}$ & \\
\hline 269 & gi|556310 & spermatid-specific & \\
\hline 270 & gi|19527104 & sphingomyelin phosphodiesterase, acid-like 3B & \\
\hline 271 & gi|19526912 & suppression of tumorigenicity 13 & \\
\hline 272 & gi|3342560 & syntenin & $\mathrm{v}$ \\
\hline 273 & gi|6678059 & syntrophin, basic 2 & \\
\hline 274 & gi| 22122345 & tetraspanin 14 & $\mathrm{v}$ \\
\hline 275 & gi|5524671 & TF-1 cell apoptosis related protein- 15 & \\
\hline 276 & gi|1167510 & TI-225 & \\
\hline 277 & gi|135667 & Tissue factor precursor (TF) (Coagulation factor III) (CD142 antigen) & \\
\hline 278 & gi|11596855 & transferrin receptor & $\mathrm{s} \mathrm{v}$ \\
\hline 279 & gi|6678359 & transketolase & \\
\hline 280 & gi|40254290 & transmembrane protein $16 \mathrm{~F}$ & \\
\hline 281 & gi|63028017 & truncated polyprotein & \\
\hline 282 & gi|4507729 & tubulin, beta 2 & $\mathrm{a}$ \\
\hline 283 & gi|7106439 & tubulin, beta 5 & $\mathrm{bcs} v$ \\
\hline 284 & gi 6755863 & tumor rejection antigen gp96 & \\
\hline 285 & gi|11230780 & tumor susceptibility gene 101 protein & $\mathrm{s}$ \\
\hline 286 & gi|16303750 & tweety homolog 2 & \\
\hline 287 & gi|15929276 & Twinfilin, actin-binding protein, homolog 1 & \\
\hline 288 & gi|16303309 & type II keratin 5 & $\mathrm{v}$ \\
\hline 289 & gi|4159806 & type II keratin subunit protein & $\mathrm{b}$ \\
\hline 290 & gi 6756039 & $\begin{array}{l}\text { tyrosine } 3 \text {-monooxygenase/tryptophan } 5 \text {-monooxygenase activation } \\
\text { protein, theta polypeptide }\end{array}$ & \\
\hline 291 & gi|5803225 & $\begin{array}{l}\text { tyrosine } 3 / \text { tryptophan } 5 \text {-monooxygenase activation protein, epsilon } \\
\text { polypeptide }\end{array}$ & \\
\hline 292 & gi $\mid 21314832$ & UDP-glucose pyrophosphorylase 2 & \\
\hline 293 & gi|148700773 & $\begin{array}{l}\text { v-ral simian leukemia viral oncogene homolog A (ras related), isoform } \\
\text { CRA_a }\end{array}$ & \\
\hline 294 & gi|11612509 & v-ral simian leukemia viral oncogene homolog B (ras related) & \\
\hline 295 & gi|30794416 & vacuolar protein sorting 36 & \\
\hline 296 & gi $\mid 29244484$ & vacuolar protein sorting 37B & \\
\hline 297 & gi|31088908 & vacuolar protein sorting $37 \mathrm{C}$ & \\
\hline 298 & gi|29164511 & vang, van gogh-like 1 & \\
\hline 299 & gi|33859662 & vesicle amine transport protein 1 homolog ( $\mathrm{T}$ californica) & \\
\hline 300 & gi|6678553 & vesicle-associated membrane protein 3 & \\
\hline 301 & gi|55451 & Y box-binbing protein & \\
\hline
\end{tabular}




\section{Appendix B}

\section{Source codes}

\section{B.1 Calculation of correlation coefficient between two channels} ImageJ Macro

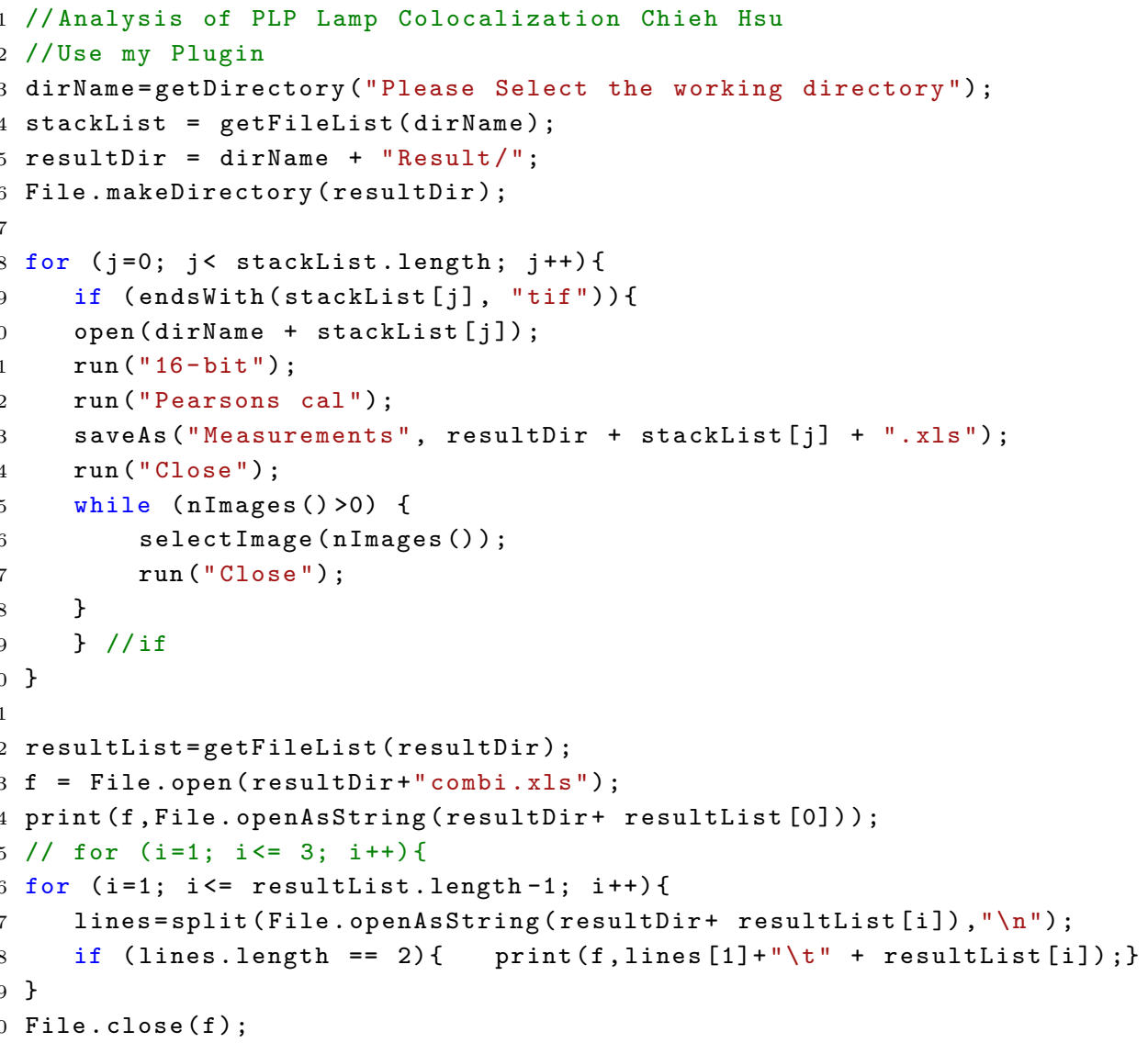




\section{ImageJ Plugin (Java)}

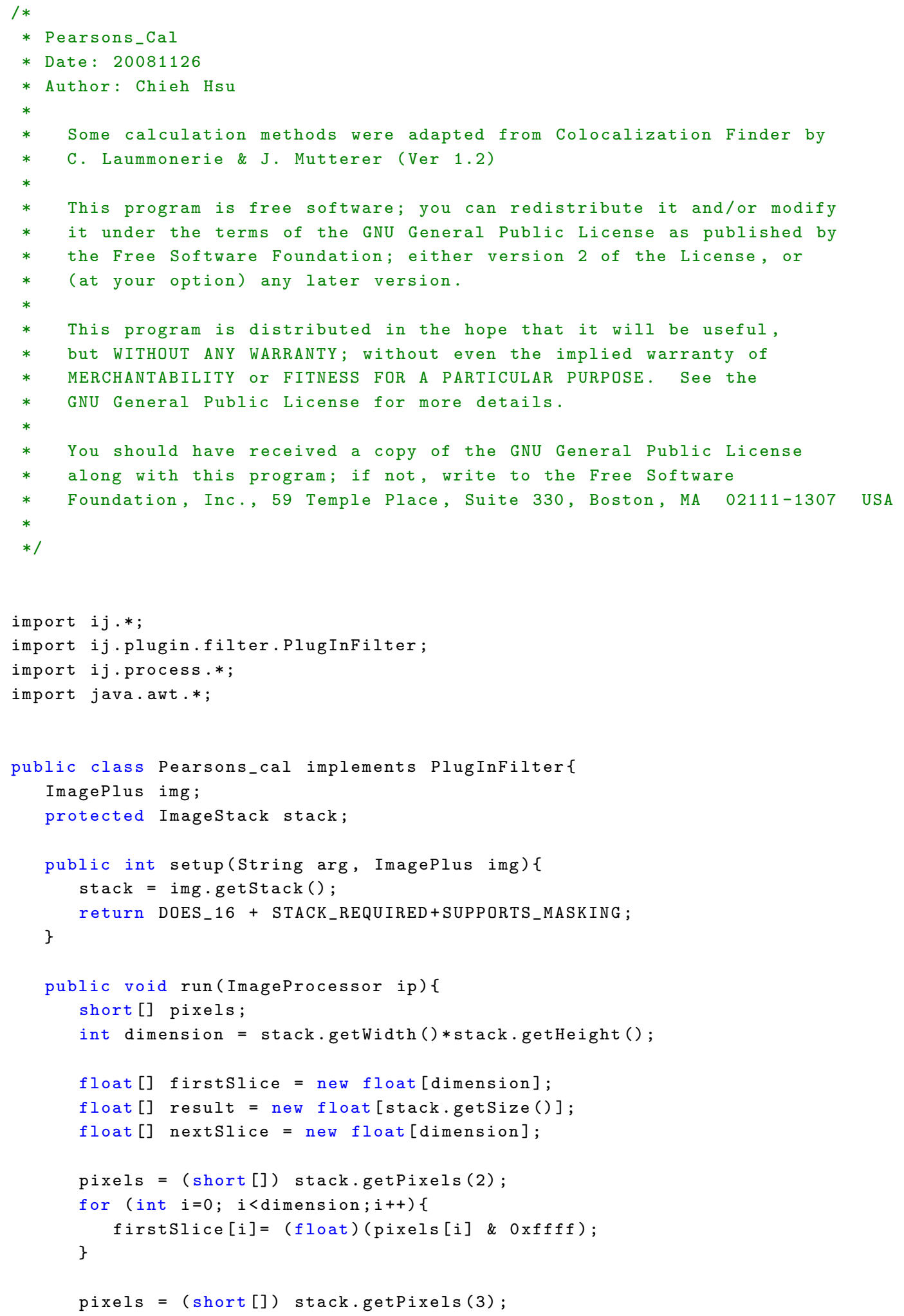




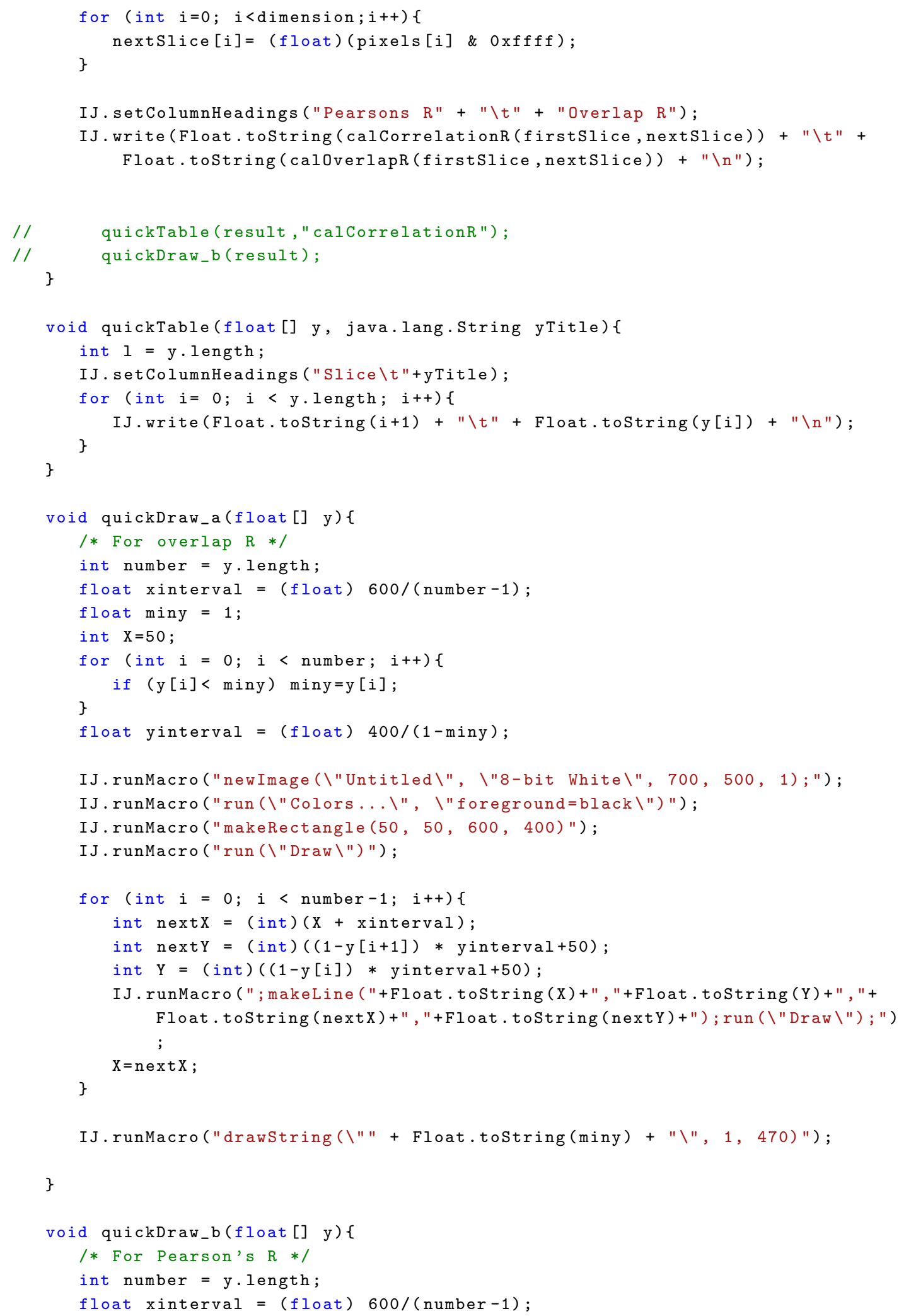




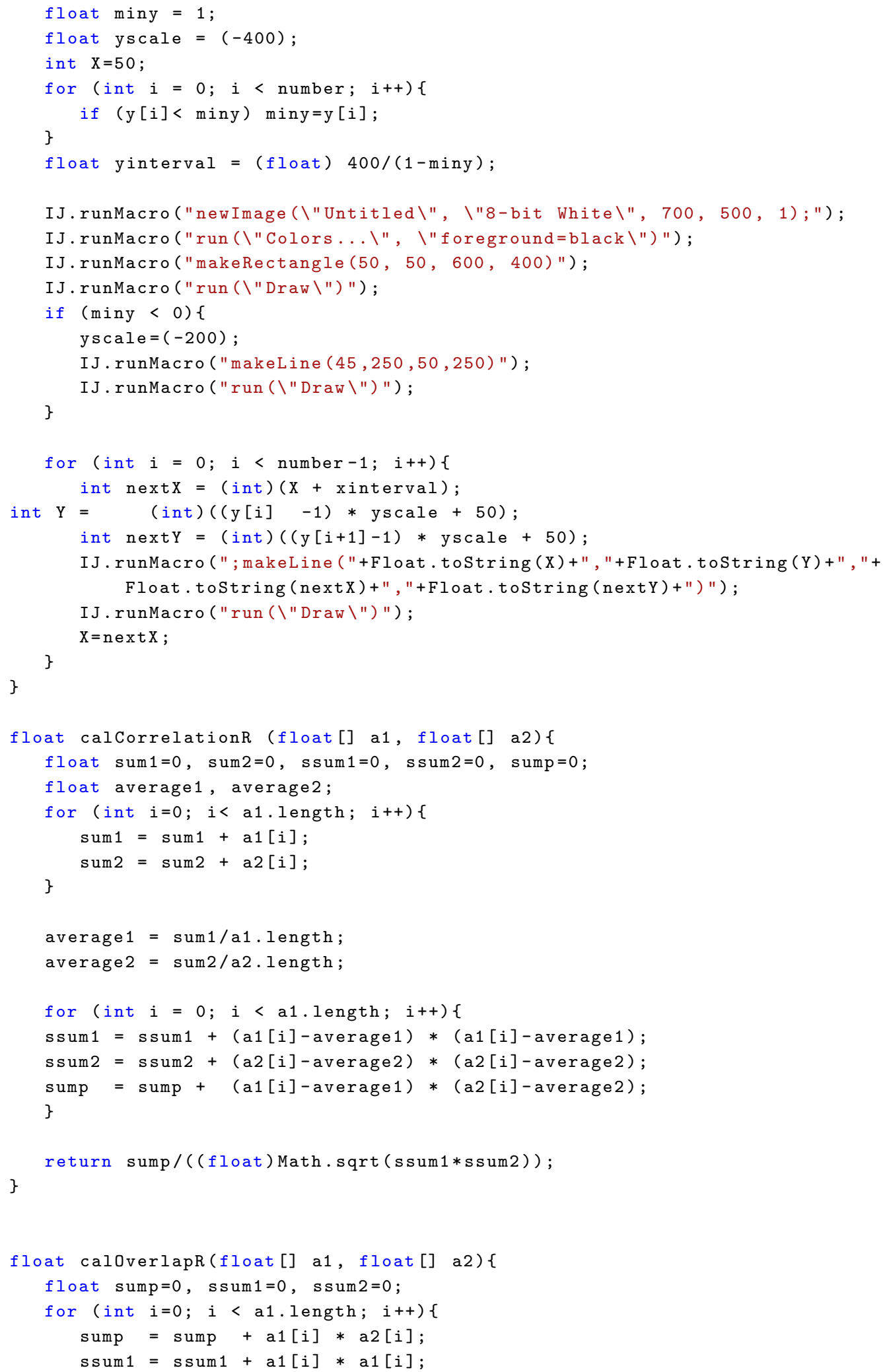




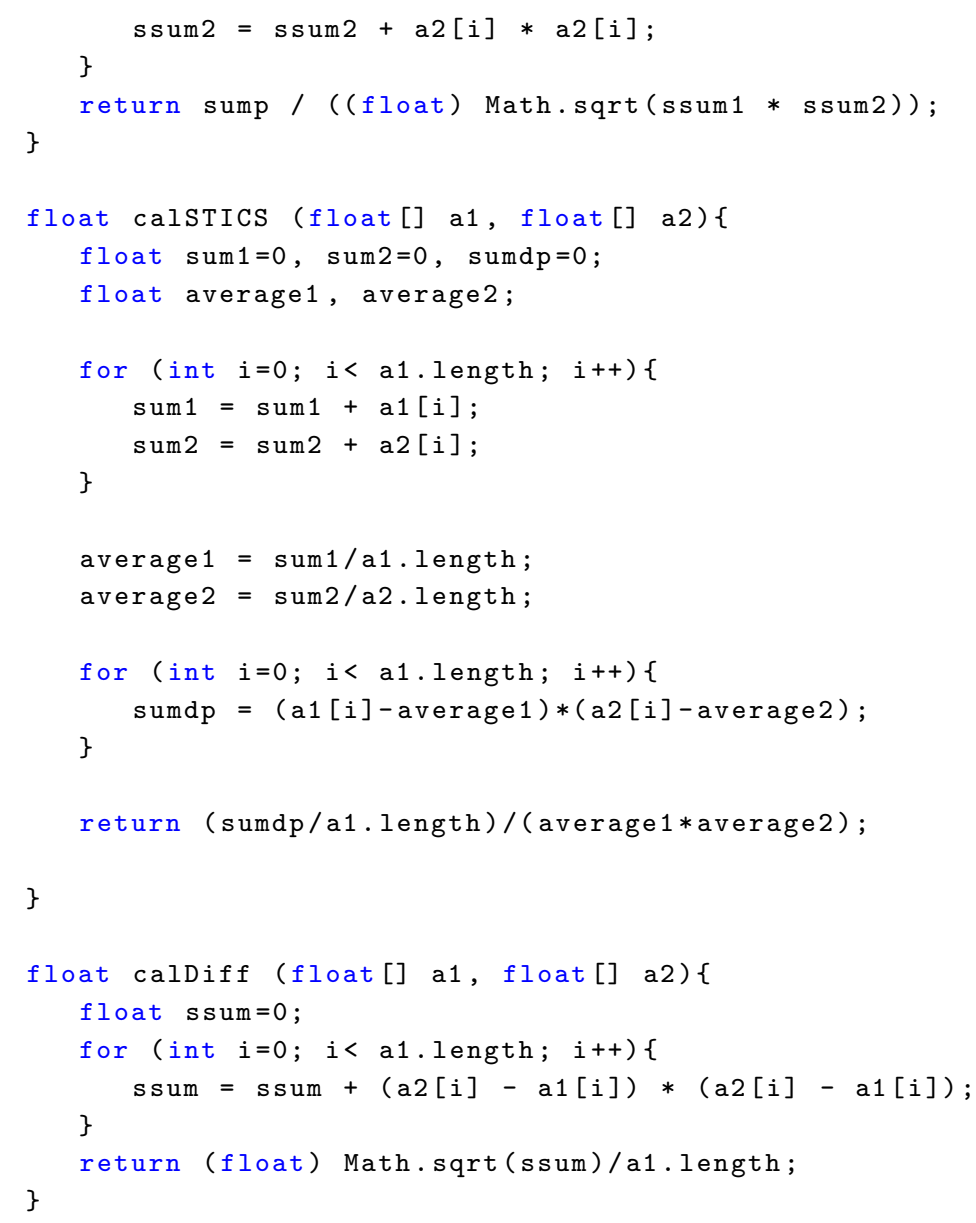




\section{B.2 Calculation of correlation coefficient within image series}

\section{ImageJ Macro}

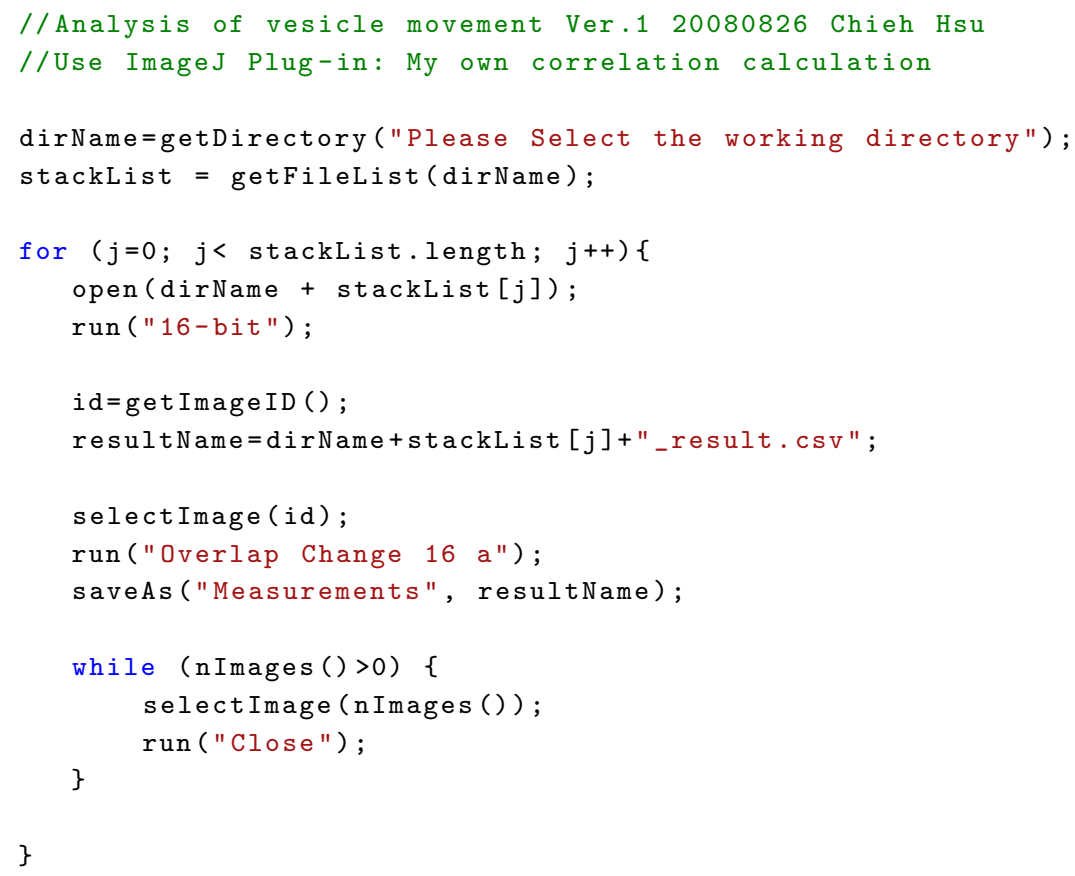

ImageJ Plugin (Java)

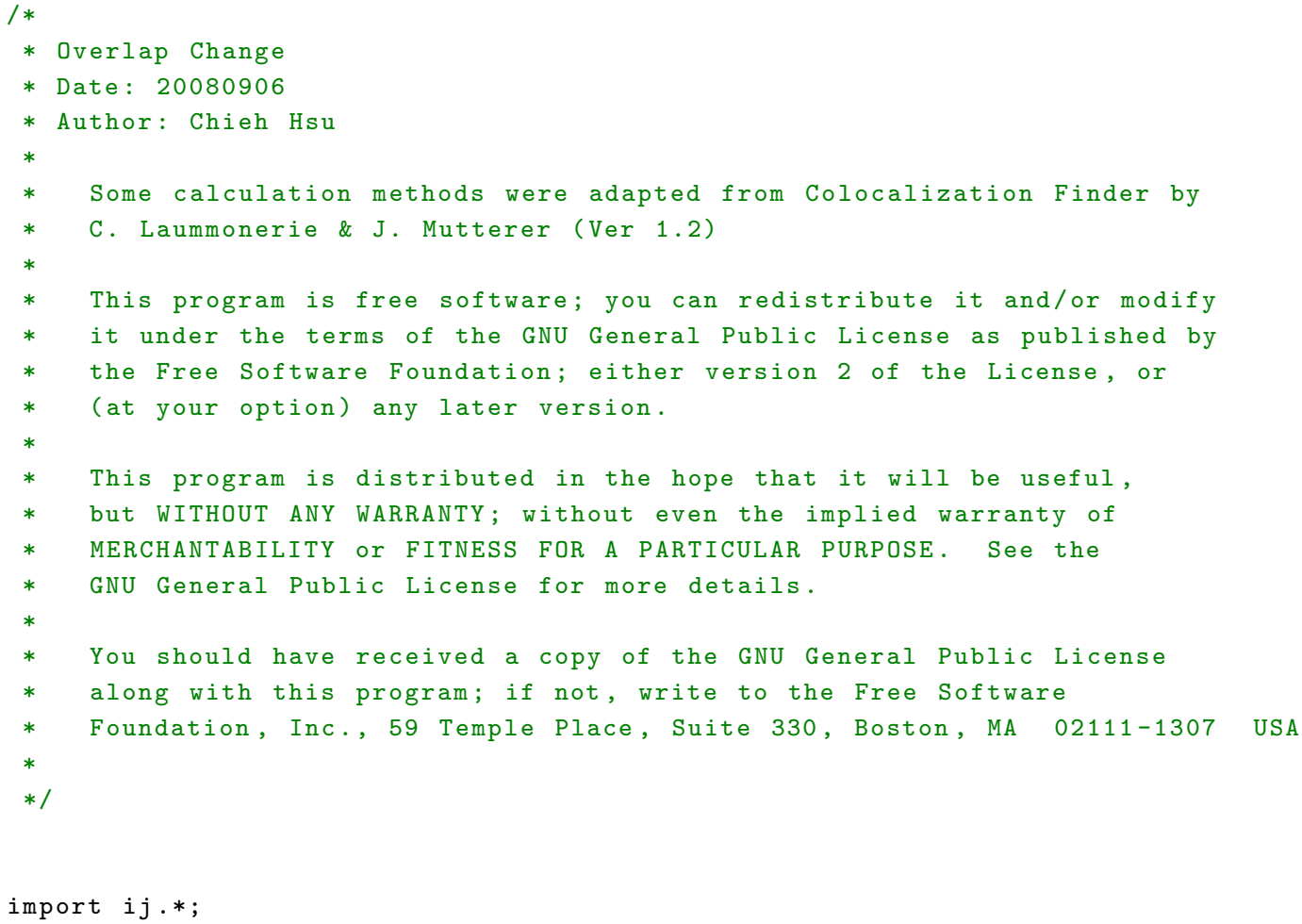




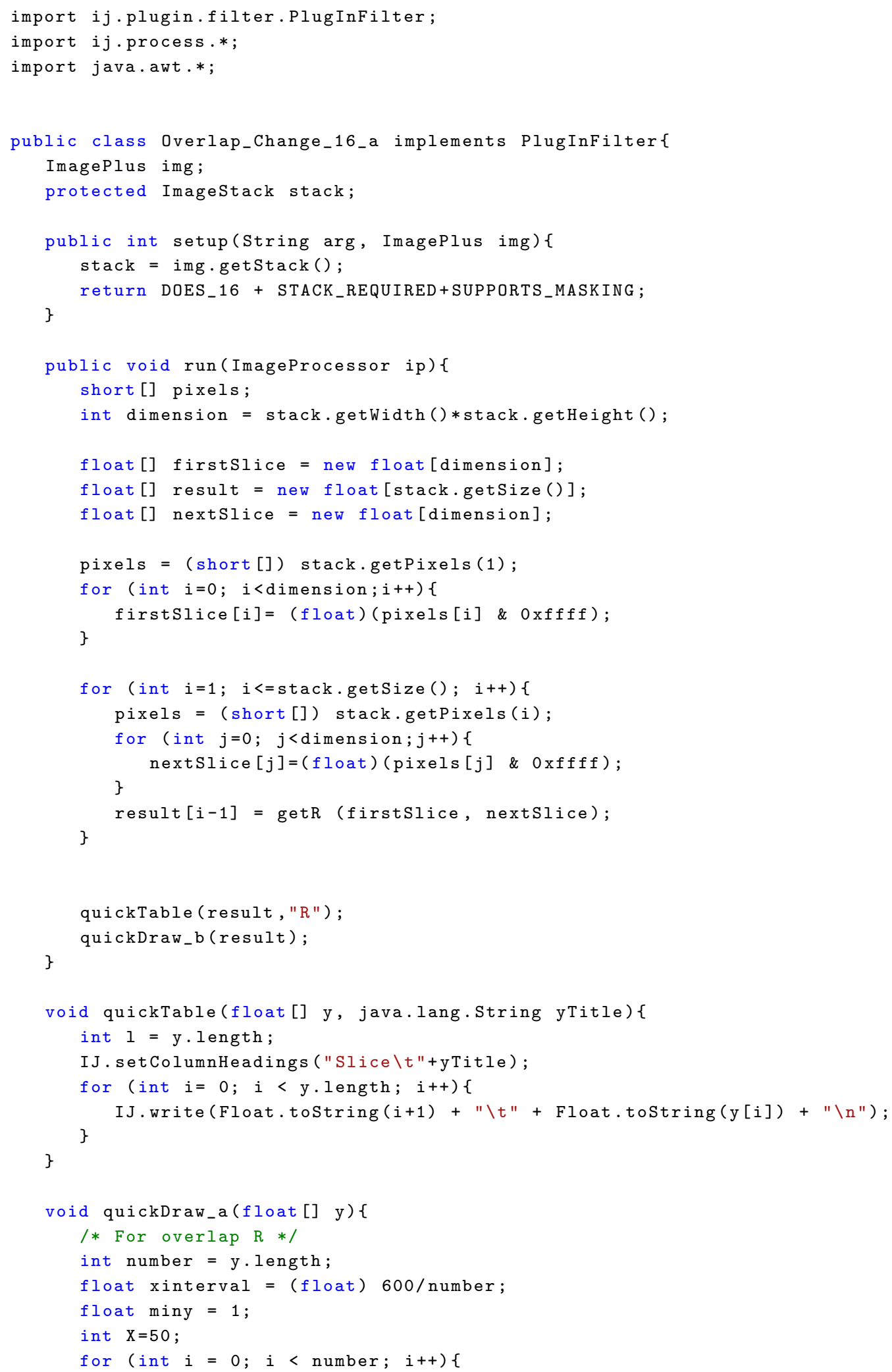




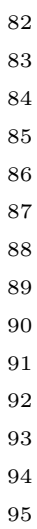

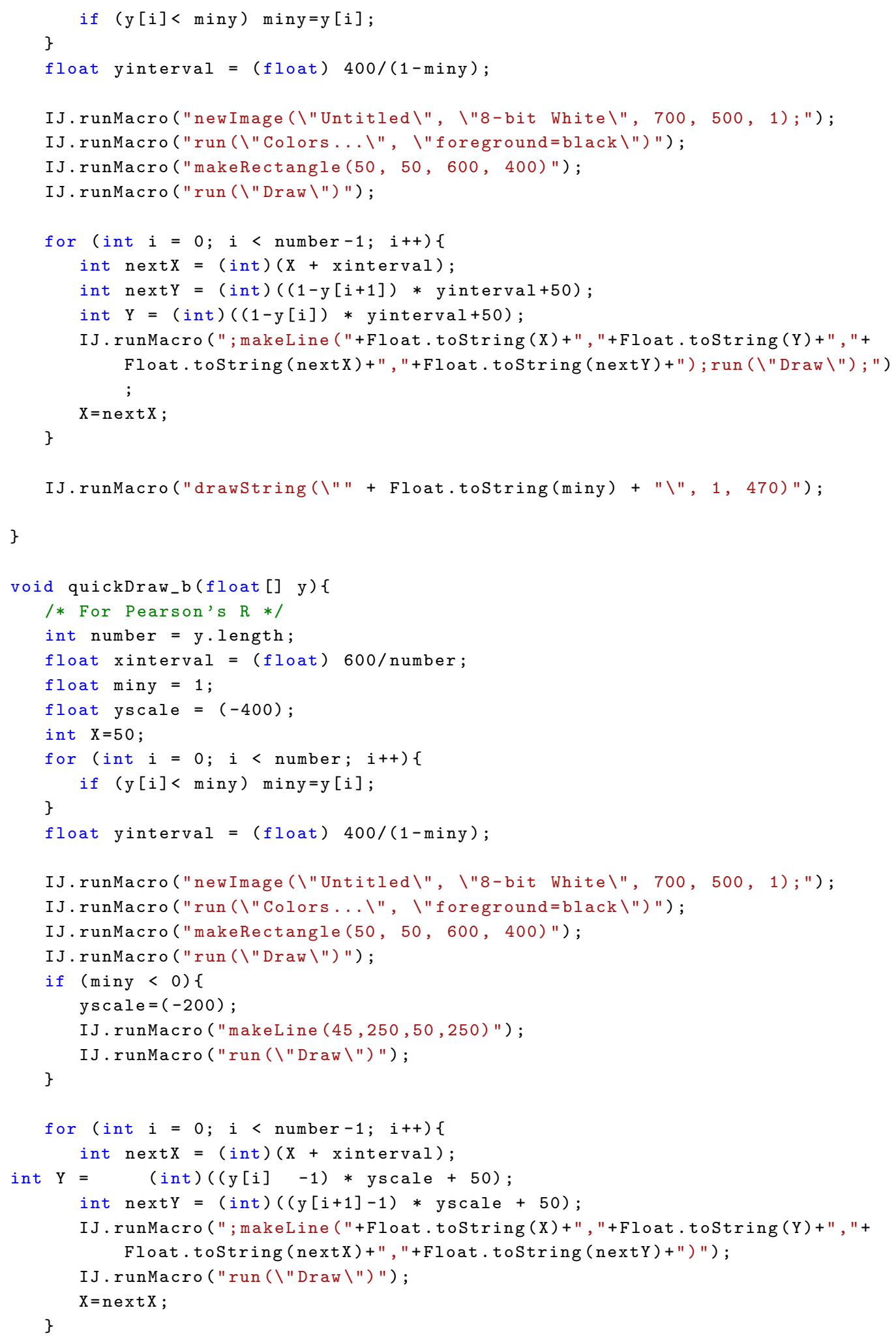




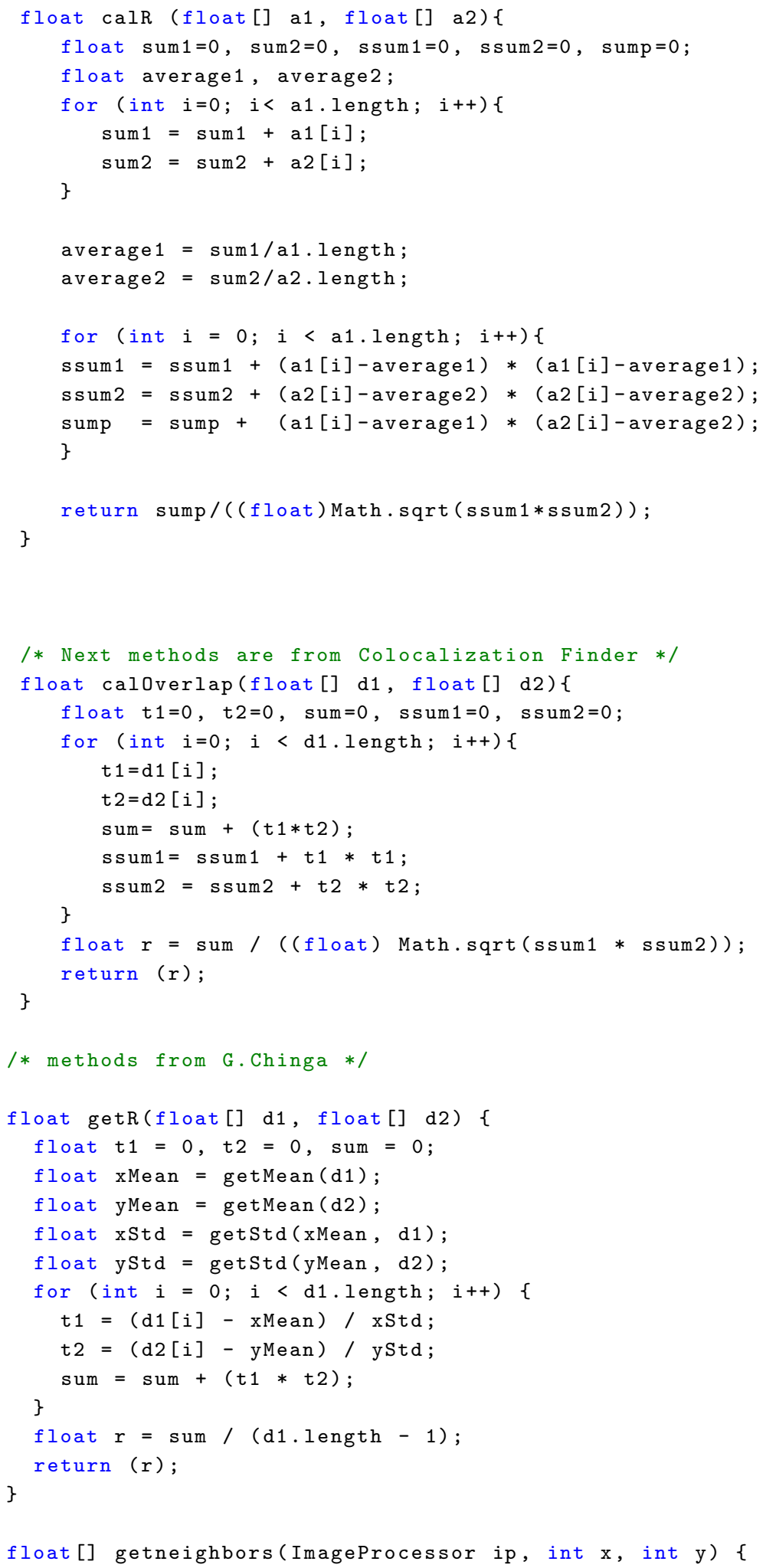




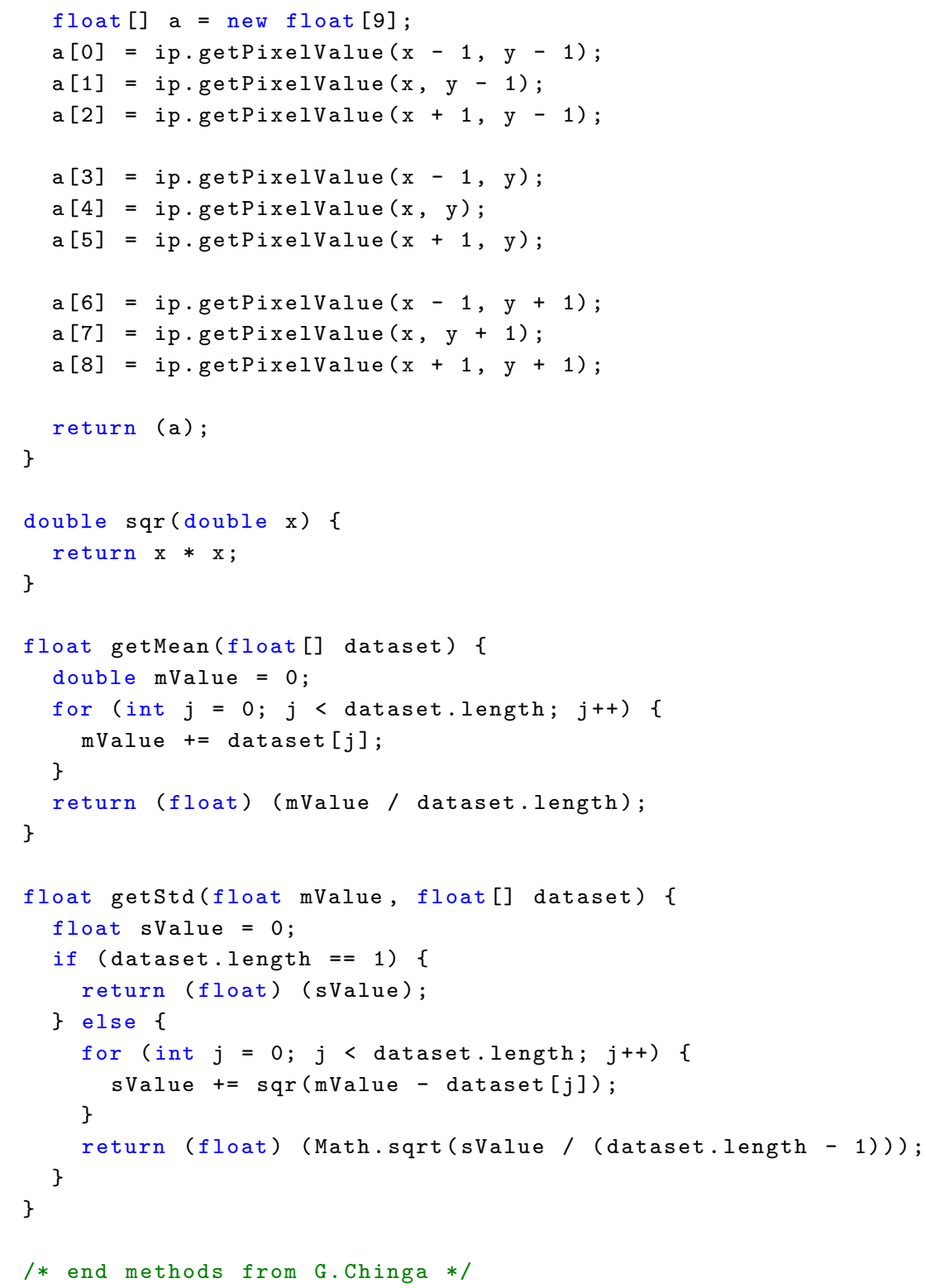




\section{Bibliography}

Abusamra, A. J., Zhong, Z., Zheng, X., Li, M., Ichim, T. E., Chin, J. L., \& Min, W.-P. (2005). Tumor exosomes expressing Fas ligand mediate CD8+ T-cell apoptosis. Blood Cells Mol Dis, $35,169-173$.

Admyre, C., Johansson, S. M., Qazi, K. R., Filén, J.-J., Lahesmaa, R., Norman, M., Neve, E. P. A., Scheynius, A., \& Gabrielsson, S. (2007). Exosomes with immune modulatory features are present in human breast milk. J Immunol, 179, 1969-1978.

Aikawa, Y. \& Martin, T. F. J. (2003). ARF6 regulates a plasma membrane pool of phosphatidylinositol(4,5)bisphosphate required for regulated exocytosis. J Cell Biol, 162, 647-659.

Alais, S., Simoes, S., Baas, D., Lehmann, S., Raposo, G., Darlix, J. L., \& Leblanc, P. (2008). Mouse neuroblastoma cells release prion infectivity associated with exosomal vesicles. Biol Cell, 100, 603-615.

Alam, S. L., Sun, J., Payne, M., Welch, B. D., Blake, B. K., Davis, D. R., Meyer, H. H., Emr, S. D., \& Sundquist, W. I. (2004). Ubiquitin interactions of NZF zinc fingers. EMBO J, 23, 1411-1421.

Altieri, S. L., Khan, A. N. H., \& Tomasi, T. B. (2004). Exosomes from plasmacytoma cells as a tumor vaccine. J Immunother, 27, 282-288.

An, Q., van Bel, A. J., \& Hückelhoven, R. (2007). Do plant cells secrete exosomes derived from multivesicular bodies? Plant Signal Behav, 2, 4-7.

Andersen, M. H., Berglund, L., Rasmussen, J. T., \& Petersen, T. E. (1997). Bovine PAS-6/7 binds alpha v beta 5 integrins and anionic phospholipids through two domains. Biochemistry, $36,5441-5446$.

Andre, F., Schartz, N. E. C., Movassagh, M., Flament, C., Pautier, P., Morice, P., Pomel, C., Lhomme, C., Escudier, B., Chevalier, T. L., Tursz, T., Amigorena, S., Raposo, G., Angevin, E., \& Zitvogel, L. (2002). Malignant effusions and immunogenic tumour-derived exosomes. Lancet, $360,295-305$.

Andrews, N. W. \& Chakrabarti, S. (2005). There's more to life than neurotransmission: the regulation of exocytosis by synaptotagmin VII. Trends Cell Biol, 15, 626-631.

André, F., Chaput, N., Schartz, N. E. C., Flament, C., Aubert, N., Bernard, J., Lemonnier, F., Raposo, G., Escudier, B., Hsu, D.-H., Tursz, T., Amigorena, S., Angevin, E., \& Zitvogel, L. 
(2004). Exosomes as potent cell-free peptide-based vaccine. I. Dendritic cell-derived exosomes transfer functional MHC class I/peptide complexes to dendritic cells. J Immunol, 172, 21262136.

Antonin, W., Holroyd, C., Fasshauer, D., Pabst, S., Mollard, G. F. V., \& Jahn, R. (2000). A SNARE complex mediating fusion of late endosomes defines conserved properties of SNARE structure and function. EMBO J, 19, 6453-6464.

Aoki, N., Jin-no, S., Nakagawa, Y., Asai, N., Arakawa, E., Tamura, N., Tamura, T., \& Matsuda, T. (2007). Identification and characterization of microvesicles secreted by 3T3-L1 adipocytes: redox- and hormone-dependent induction of milk fat globule-epidermal growth factor 8-associated microvesicles. Endocrinology, 148, 3850-3862.

Aubin, I., Adams, C. P., Opsahl, S., Septier, D., Bishop, C. E., Auge, N., Salvayre, R., NegreSalvayre, A., Goldberg, M., Guénet, J.-L., \& Poirier, C. (2005). A deletion in the gene encoding sphingomyelin phosphodiesterase $3(\mathrm{Smpd} 3)$ results in osteogenesis and dentinogenesis imperfecta in the mouse. Nat Genet, 37, 803-805.

Babst, M., Odorizzi, G., Estepa, E. J., \& Emr, S. D. (2000). Mammalian tumor susceptibility gene 101 (TSG101) and the yeast homologue, Vps23p, both function in late endosomal trafficking. Traffic, 1, 248-258.

Bache, K. G., Brech, A., Mehlum, A., \& Stenmark, H. (2003). Hrs regulates multivesicular body formation via ESCRT recruitment to endosomes. J Cell Biol, 162, 435-442.

Bache, K. G., Stuffers, S., Malerød, L., Slagsvold, T., Raiborg, C., Lechardeur, D., Wälchli, S., Lukacs, G. L., Brech, A., \& Stenmark, H. (2006). The ESCRT-III subunit hVps24 is required for degradation but not silencing of the epidermal growth factor receptor. Mol Biol Cell, 17, $2513-2523$.

Baldys, A. \& Raymond, J. R. (2009). Critical role of ESCRT machinery in EGFR recycling. Biochemistry, 48, 9321-9323.

Barbero, P., Bittova, L., \& Pfeffer, S. R. (2002). Visualization of Rab9-mediated vesicle transport from endosomes to the trans-Golgi in living cells. J Cell Biol, 156, 511-518.

Barile, M., Pisitkun, T., Yu, M.-J., Chou, C.-L., Verbalis, M. J., Shen, R.-F., \& Knepper, M. A. (2005). Large scale protein identification in intracellular aquaporin-2 vesicles from renal inner medullary collecting duct. Mol Cell Proteomics, 4, 1095-1106.

Barres, B. A. (2008). The mystery and magic of glia: a perspective on their roles in health and disease. Neuron, 60, 430-440.

Barriere, H., Nemes, C., Du, K., \& Lukacs, G. L. (2007). Plasticity of polyubiquitin recognition as lysosomal targeting signals by the endosomal sorting machinery. Mol Biol Cell, 18, 3952-3965.

Baumann, N. \& Pham-Dinh, D. (2001). Biology of oligodendrocyte and myelin in the mammalian central nervous system. Physiol Rev, 81, 871-927. 
Baumgart, T., Hess, S. T., \& Webb, W. W. (2003). Imaging coexisting fluid domains in biomembrane models coupling curvature and line tension. Nature, 425, 821-824.

Berson, J. F., Harper, D. C., Tenza, D., Raposo, G., \& Marks, M. S. (2001). Pmel17 initiates premelanosome morphogenesis within multivesicular bodies. Mol Biol Cell, 12, 3451-3464.

Berson, J. F., Theos, A. C., Harper, D. C., Tenza, D., Raposo, G., \& Marks, M. S. (2003). Proprotein convertase cleavage liberates a fibrillogenic fragment of a resident glycoprotein to initiate melanosome biogenesis. J Cell Biol, 161, 521-533.

Bishop, N. \& Woodman, P. (2000). ATPase-defective mammalian VPS4 localizes to aberrant endosomes and impairs cholesterol trafficking. Mol Biol Cell, 11, 227-239.

Boucheix, C. \& Rubinstein, E. (2001). Tetraspanins. Cell Mol Life Sci, 58, 1189-1205.

Bradford, M. M. (1976). A rapid and sensitive method for the quantitation of microgram quantities of protein utilizing the principle of protein-dye binding. Anal Biochem, 72, 248-254.

Braulke, T. \& Bonifacino, J. S. (2009). Sorting of lysosomal proteins. Biochim Biophys Acta, 1793, 605-614.

Brown, D. A. \& London, E. (2000). Structure and function of sphingolipid- and cholesterol-rich membrane rafts. J Biol Chem, 275, 17221-17224.

Brown, F. D., Rozelle, A. L., Yin, H. L., Balla, T., \& Donaldson, J. G. (2001). Phosphatidylinositol 4,5-bisphosphate and Arf6-regulated membrane traffic. J Cell Biol, 154, 1007-1017.

Brown, W. J., Chambers, K., \& Doody, A. (2003). Phospholipase A2 (PLA2) enzymes in membrane trafficking: mediators of membrane shape and function. Traffic, 4, 214-221.

Burkhardt, J. K., McIlvain, J. M., Sheetz, M. P., \& Argon, Y. (1993). Lytic granules from cytotoxic $\mathrm{T}$ cells exhibit kinesin-dependent motility on microtubules in vitro. J Cell Sci, 104 ( Pt 1), 151162.

Caby, M.-P., Lankar, D., Vincendeau-Scherrer, C., Raposo, G., \& Bonnerot, C. (2005). Exosomallike vesicles are present in human blood plasma. Int Immunol, 17, 879-887.

Carlton, J. G., Agromayor, M., \& Martin-Serrano, J. (2008). Differential requirements for Alix and ESCRT-III in cytokinesis and HIV-1 release. Proc Natl Acad Sci U S A, 105, 10541-10546.

Carlton, J. G. \& Martin-Serrano, J. (2007). Parallels between cytokinesis and retroviral budding: a role for the ESCRT machinery. Science, 316, 1908-1912.

Carlton, J. G. \& Martin-Serrano, J. (2009). The ESCRT machinery: new functions in viral and cellular biology. Biochem Soc Trans, 37, 195-199.

Carter, R. E. \& Sorkin, A. (1998). Endocytosis of functional epidermal growth factor receptor-green fluorescent protein chimera. J Biol Chem, 273, 35000-35007.

Chavrier, P., Gorvel, J. P., Stelzer, E., Simons, K., Gruenberg, J., \& Zerial, M. (1991). Hypervariable C-terminal domain of rab proteins acts as a targeting signal. Nature, 353, 769-772. 
Christoforidis, S., McBride, H. M., Burgoyne, R. D., \& Zerial, M. (1999). The Rab5 effector EEA1 is a core component of endosome docking. Nature, 397, 621-625.

Clarke, C. J. \& Hannun, Y. A. (2006). Neutral sphingomyelinases and nSMase2: bridging the gaps. Biochim Biophys Acta, 1758, 1893-1901.

Clarke, C. J., Truong, T.-G., \& Hannun, Y. A. (2007). Role for neutral sphingomyelinase-2 in tumor necrosis factor alpha-stimulated expression of vascular cell adhesion molecule-1 (VCAM) and intercellular adhesion molecule-1 (ICAM) in lung epithelial cells: p38 MAPK is an upstream regulator of nSMase2. J Biol Chem, 282, 1384-1396.

Clayton, A., Mitchell, J. P., Court, J., Linnane, S., Mason, M. D., \& Tabi, Z. (2008). Human tumor-derived exosomes down-modulate NKG2D expression. J Immunol, 180, 7249-7258.

Clayton, A., Mitchell, J. P., Court, J., Mason, M. D., \& Tabi, Z. (2007). Human tumor-derived exosomes selectively impair lymphocyte responses to interleukin-2. Cancer Res, 67, 7458-7466.

Conde-Vancells, J., Rodriguez-Suarez, E., Embade, N., Gil, D., Matthiesen, R., Valle, M., Elortza, F., Lu, S., Mato, J., \& Falcon-Perez, J. (2008). Characterization and Comprehensive Proteome Profiling of Exosomes Secreted by Hepatocytes. J Proteome Res, 7, 5157-5166.

Court, F. A., Hendriks, W. T. J., Macgillavry, H. D., Alvarez, J., \& van Minnen, J. (2008). Schwann cell to axon transfer of ribosomes: toward a novel understanding of the role of glia in the nervous system. J Neurosci, 28, 11024-11029.

Cremesti, A. E., Goni, F. M., \& Kolesnick, R. (2002). Role of sphingomyelinase and ceramide in modulating rafts: do biophysical properties determine biologic outcome? FEBS Lett, 531, $47-53$.

Crosetto, N., Tikkanen, R., \& Dikic, I. (2005). Oncogenic breakdowns in endocytic adaptor proteins. FEBS Lett, 579, 3231-3238.

Dai, S., Wei, D., Wu, Z., Zhou, X., Wei, X., Huang, H., \& Li, G. (2008). Phase I clinical trial of autologous ascites-derived exosomes combined with GM-CSF for colorectal cancer. Mol Ther, $16,782-790$.

Davis, J. Q., Dansereau, D., Johnstone, R. M., \& Bennett, V. (1986). Selective externalization of an ATP-binding protein structurally related to the clathrin-uncoating ATPase/heat shock protein in vesicles containing terminal transferrin receptors during reticulocyte maturation. J Biol Chem, 261, 15368-15371.

de Gassart, A., Geminard, C., Fevrier, B., Raposo, G., \& Vidal, M. (2003). Lipid raft-associated protein sorting in exosomes. Blood, 102, 4336-4344.

Dell'Angelica, E. C., Mullins, C., Caplan, S., \& Bonifacino, J. S. (2000). Lysosome-related organelles. FASEB J, 14, 1265-1278.

Denzer, K., Kleijmeer, M. J., Heijnen, H. F., Stoorvogel, W., \& Geuze, H. J. (2000). Exosome: from internal vesicle of the multivesicular body to intercellular signaling device. J Cell Sci, 113 Pt 19, 3365-3374. 
Duan, R.-D. (2006). Alkaline sphingomyelinase: an old enzyme with novel implications. Biochim Biophys Acta, 1761, 281-291.

Dumas, J. J., Zhu, Z., Connolly, J. L., \& Lambright, D. G. (1999). Structural basis of activation and GTP hydrolysis in Rab proteins. Structure, 7, 413-423.

Escola, J. M., Kleijmeer, M. J., Stoorvogel, W., Griffith, J. M., Yoshie, O., \& Geuze, H. J. (1998). Selective enrichment of tetraspan proteins on the internal vesicles of multivesicular endosomes and on exosomes secreted by human B-lymphocytes. J Biol Chem, 273, 20121-20127.

Escudier, B., Dorval, T., Chaput, N., André, F., Caby, M.-P., Novault, S., Flament, C., Leboulaire, C., Borg, C., Amigorena, S., Boccaccio, C., Bonnerot, C., Dhellin, O., Movassagh, M., Piperno, S., Robert, C., Serra, V., Valente, N., Pecq, J.-B. L., Spatz, A., Lantz, O., Tursz, T., Angevin, E., \& Zitvogel, L. (2005). Vaccination of metastatic melanoma patients with autologous dendritic cell (DC) derived-exosomes: results of thefirst phase I clinical trial. J Transl Med, 3, 10.

Fader, C. M., Sánchez, D. G., Mestre, M. B., \& Colombo, M. I. (2009). TI-VAMP/VAMP7 and VAMP3/cellubrevin: two v-SNARE proteins involved in specific steps of the autophagy/multivesicular body pathways. Biochim Biophys Acta.

Falguières, T., Luyet, P.-P., Bissig, C., Scott, C. C., Velluz, M.-C., \& Gruenberg, J. (2008). In vitro budding of intralumenal vesicles into late endosomes is regulated by Alix and Tsg101. Mol Biol Cell, 19, 4942-4955.

Falguières, T., Luyet, P.-P., \& Gruenberg, J. (2009). Molecular assemblies and membrane domains in multivesicular endosome dynamics. Exp Cell Res, 315, 1567-1573.

Fang, Y., Wu, N., Gan, X., Yan, W., Morrell, J. C., \& Gould, S. J. (2007). Higher-order oligomerization targets plasma membrane proteins and HIV gag to exosomes. PLoS Biol, 5, e158.

Fauré, J., Lachenal, G., Court, M., Hirrlinger, J., Chatellard-Causse, C., Blot, B., Grange, J., Schoehn, G., Goldberg, Y., Boyer, V., Kirchhoff, F., Raposo, G., Garin, J., \& Sadoul, R. (2006). Exosomes are released by cultured cortical neurones. Mol Cell Neurosci, 31, 642-648.

Feldmann, J., Callebaut, I., Raposo, G., Certain, S., Bacq, D., Dumont, C., Lambert, N., OuachéeChardin, M., Chedeville, G., Tamary, H., Minard-Colin, V., Vilmer, E., Blanche, S., Deist, F. L., Fischer, A., \& de Saint Basile, G. (2003). Munc13-4 is essential for cytolytic granules fusion and is mutated in a form of familial hemophagocytic lymphohistiocytosis (FHL3). Cell, 115, $461-473$.

Fevrier, B., Vilette, D., Archer, F., Loew, D., Faigle, W., Vidal, M., Laude, H., \& Raposo, G. (2004). Cells release prions in association with exosomes. Proc Natl Acad Sci U S A, 101, 9683-9688.

Fisher, R. D., Chung, H.-Y., Zhai, Q., Robinson, H., Sundquist, W. I., \& Hill, C. P. (2007). Structural and biochemical studies of ALIX/AIP1 and its role in retrovirus budding. Cell, 128, $841-852$. 
Fitzner, D., Schneider, A., Kippert, A., Möbius, W., Willig, K. I., Hell, S. W., Bunt, G., Gaus, K., \& Simons, M. (2006). Myelin basic protein-dependent plasma membrane reorganization in the formation of myelin. EMBO J, 25, 5037-5048.

Fox, A. S. \& Yoon, S. B. (1970). DNA-induced transformation in Drosophila: locus-specificity and the establishment of transformed stocks. Proc Natl Acad Sci U S A, 67, 1608-1615.

Freed, E. O. (1998). HIV-1 gag proteins: diverse functions in the virus life cycle. Virology, 251, $1-15$.

Fuchs, E., Haas, A. K., Spooner, R. A., ichiro Yoshimura, S., Lord, J. M., \& Barr, F. A. (2007). Specific Rab GTPase-activating proteins define the Shiga toxin and epidermal growth factor uptake pathways. J Cell Biol, 177, 1133-1143.

Fukuda, M. (2002). Synaptotagmin-like protein (Slp) homology domain 1 of Slac2-a/melanophilin is a critical determinant of GTP-dependent specific binding to Rab27A. J Biol Chem, 277, 40118-40124.

Fukuda, M. (2006). Rab27 and its effectors in secretory granule exocytosis: a novel docking machinery composed of a Rab27.effector complex. Biochem Soc Trans, 34, 691-695.

Fukuda, M., Kanno, E., Saegusa, C., Ogata, Y., \& Kuroda, T. S. (2002). Slp4-a/granuphilin-a regulates dense-core vesicle exocytosis in PC12 cells. J Biol Chem, 277, 39673-39678.

Futter, C. E., Pearse, A., Hewlett, L. J., \& Hopkins, C. R. (1996). Multivesicular endosomes containing internalized EGF-EGF receptor complexes mature and then fuse directly with lysosomes. J Cell Biol, 132, 1011-1023.

Garcia-Saez, I., Tcherniuk, S., \& Kozielski, F. (2006). The structure of human neuronal Rab6B in the active and inactive form. Acta Crystallogr D Biol Crystallogr, 62, 725-733.

Garrus, J. E., von Schwedler, U. K., Pornillos, O. W., Morham, S. G., Zavitz, K. H., Wang, H. E., Wettstein, D. A., Stray, K. M., Côté, M., Rich, R. L., Myszka, D. G., \& Sundquist, W. I. (2001). Tsg101 and the vacuolar protein sorting pathway are essential for HIV-1 budding. Cell, 107, $55-65$.

Gaullier, J. M., Simonsen, A., D’Arrigo, A., Bremnes, B., Stenmark, H., \& Aasland, R. (1998). FYVE fingers bind PtdIns(3)P. Nature, 394, 432-433.

Gelderblom, H. R., Ozel, M., \& Pauli, G. (1989). Morphogenesis and morphology of HIV. Structurefunction relations. Arch Virol, 106, 1-13.

Gesierich, S., Berezovskiy, I., Ryschich, E., \& Zöller, M. (2006). Systemic induction of the angiogenesis switch by the tetraspanin D6.1A/CO-029. Cancer Res, 66, 7083-7094.

Ghazi-Tabatabai, S., Obita, T., Pobbati, A. V., Perisic, O., Samson, R. Y., Bell, S. D., \& Williams, R. L. (2009). Evolution and assembly of ESCRTs. Biochem Soc Trans, 37, 151-155. 
Gheysen, D., Jacobs, E., de Foresta, F., Thiriart, C., Francotte, M., Thines, D., \& Wilde, M. D. (1989). Assembly and release of HIV-1 precursor Pr55gag virus-like particles from recombinant baculovirus-infected insect cells. Cell, 59, 103-112.

Ghosh, P., Dahms, N. M., \& Kornfeld, S. (2003). Mannose 6-phosphate receptors: new twists in the tale. Nat Rev Mol Cell Biol, 4, 202-212.

Goila-Gaur, R., Demirov, D. G., Orenstein, J. M., Ono, A., \& Freed, E. O. (2003). Defects in human immunodeficiency virus budding and endosomal sorting induced by TSG101 overexpression. J Virol, 77, 6507-6519.

Goishi, K., Mizuno, K., Nakanishi, H., \& Sasaki, T. (2004). Involvement of Rab27 in antigeninduced histamine release from rat basophilic leukemia $2 \mathrm{H} 3$ cells. Biochem Biophys Res Commun, 324, 294-301.

Gold, R., Hartung, H. P., \& Toyka, K. V. (2000). Animal models for autoimmune demyelinating disorders of the nervous system. Mol Med Today, 6, 88-91.

Gould, G. W. \& Lippincott-Schwartz, J. (2009). New roles for endosomes: from vesicular carriers to multi-purpose platforms. Nat Rev Mol Cell Biol, 10, 287-292.

Gould, S. J., Booth, A. M., \& Hildreth, J. E. K. (2003). The Trojan exosome hypothesis. Proc Natl Acad Sci U S A, 100, 10592-10597.

Grant, B. D. \& Donaldson, J. G. (2009). Pathways and mechanisms of endocytic recycling. Nat Rev Mol Cell Biol, 10, 597-608.

Greco, V., Hannus, M., \& Eaton, S. (2001). Argosomes: a potential vehicle for the spread of morphogens through epithelia. Cell, 106, 633-645.

Grewal, T. \& Enrich, C. (2009). Annexins - modulators of EGF receptor signalling and trafficking. Cell Signal.

Griffiths, I. R., Schneider, A., Anderson, J., \& Nave, K. A. (1995). Transgenic and natural mouse models of proteolipid protein (PLP)-related dysmyelination and demyelination. Brain Pathol, $5,275-281$.

Griffiths, R. E., Heesom, K. J., \& Anstee, D. J. (2007). Normal prion protein trafficking in cultured human erythroblasts. Blood, 110, 4518-4525.

Gruenberg, J. \& van der Goot, F. G. (2006). Mechanisms of pathogen entry through the endosomal compartments. Nat Rev Mol Cell Biol, 7, 495-504.

Guo, W., Roth, D., Walch-Solimena, C., \& Novick, P. (1999). The exocyst is an effector for Sec4p, targeting secretory vesicles to sites of exocytosis. EMBO J, 18, 1071-1080.

Haas, A. K., Fuchs, E., Kopajtich, R., \& Barr, F. A. (2005). A GTPase-activating protein controls Rab5 function in endocytic trafficking. Nat Cell Biol, 7, 887-893. 
Haas, A. K., ichiro Yoshimura, S., Stephens, D. J., Preisinger, C., Fuchs, E., \& Barr, F. A. (2007). Analysis of GTPase-activating proteins: Rab1 and Rab43 are key Rabs required to maintain a functional Golgi complex in human cells. J Cell Sci, 120, 2997-3010.

Halperin, W. \& Jensen, W. A. (1967). Ultrastructural changes during growth and embryogenesis in carrot cell cultures. J Ultrastruct Res, 18, 428-443.

Hanson, P. I., Roth, R., Lin, Y., \& Heuser, J. E. (2008). Plasma membrane deformation by circular arrays of ESCRT-III protein filaments. J Cell Biol, 180, 389-402.

Hao, S., Bai, O., Yuan, J., Qureshi, M., \& Xiang, J. (2006). Dendritic cell-derived exosomes stimulate stronger CD8+ CTL responses and antitumor immunity than tumor cell-derived exosomes. Cell Mol Immunol, 3, 205-211.

Harding, C., Heuser, J., \& Stahl, P. (1983). Receptor-mediated endocytosis of transferrin and recycling of the transferrin receptor in rat reticulocytes. J Cell Biol, 97, 329-339.

Harper, D. C., Theos, A. C., Herman, K. E., Tenza, D., Raposo, G., \& Marks, M. S. (2008). Premelanosome amyloid-like fibrils are composed of only golgi-processed forms of Pmel17 that have been proteolytically processed in endosomes. J Biol Chem, 283, 2307-2322.

Heijnen, H. F., Schiel, A. E., Fijnheer, R., Geuze, H. J., \& Sixma, J. J. (1999). Activated platelets release two types of membrane vesicles: microvesicles by surface shedding and exosomes derived from exocytosis of multivesicular bodies and alpha-granules. Blood, 94, 3791-3799.

Hess, C., Sadallah, S., Hefti, A., Landmann, R., \& Schifferli, J. A. (1999). Ectosomes released by human neutrophils are specialized functional units. J Immunol, 163, 4564-4573.

Hierro, A., Sun, J., Rusnak, A. S., Kim, J., Prag, G., Emr, S. D., \& Hurley, J. H. (2004). Structure of the ESCRT-II endosomal trafficking complex. Nature, 431, 221-225.

Hofmann, K. \& Falquet, L. (2001). A ubiquitin-interacting motif conserved in components of the proteasomal and lysosomal protein degradation systems. Trends Biochem Sci, 26, 347-350.

Hofmann, K., Tomiuk, S., Wolff, G., \& Stoffel, W. (2000). Cloning and characterization of the mammalian brain-specific, Mg2+-dependent neutral sphingomyelinase. Proc Natl Acad Sci U S A, $97,5895-5900$.

Holopainen, J. M., Angelova, M. I., \& Kinnunen, P. K. (2000). Vectorial budding of vesicles by asymmetrical enzymatic formation of ceramide in giant liposomes. Biophys J, 78, 830-838.

Holt, O. J., Gallo, F., \& Griffiths, G. M. (2006). Regulating secretory lysosomes. J Biochem, 140, $7-12$.

Hood, J. L., Pan, H., Lanza, G. M., \& Wickline, S. A. (2009). Paracrine induction of endothelium by tumor exosomes. Lab Invest.

Hoorn, E. J., Pisitkun, T., Zietse, R., Gross, P., Frokiaer, J., Wang, N. S., Gonzales, P. A., Star, R. A., \& Knepper, M. A. (2005). Prospects for urinary proteomics: exosomes as a source of urinary biomarkers. Nephrology (Carlton), 10, 283-290. 
Huang, F., Kirkpatrick, D., Jiang, X., Gygi, S., \& Sorkin, A. (2006). Differential regulation of EGF receptor internalization and degradation by multiubiquitination within the kinase domain. Mol Cell, 21, 737-748.

Huang, S., Lifshitz, L. M., Jones, C., Bellve, K. D., Standley, C., Fonseca, S., Corvera, S., Fogarty, K. E., \& Czech, M. P. (2007). Insulin stimulates membrane fusion and GLUT4 accumulation in clathrin coats on adipocyte plasma membranes. Mol Cell Biol, 27, 3456-3469.

Hunter, M. P., Ismail, N., Zhang, X., Aguda, B. D., Lee, E. J., Yu, L., Xiao, T., Schafer, J., Lee, M.-L. T., Schmittgen, T. D., Nana-Sinkam, S. P., Jarjoura, D., \& Marsh, C. B. (2008). Detection of microRNA expression in human peripheral blood microvesicles. PLoS One, 3, e3694.

Hurley, J. H. \& Emr, S. D. (2006). The ESCRT complexes: structure and mechanism of a membrane-trafficking network. Annu Rev Biophys Biomol Struct, 35, 277-298.

Iero, M., Valenti, R., Huber, V., Filipazzi, P., Parmiani, G., Fais, S., \& Rivoltini, L. (2008). Tumour-released exosomes and their implications in cancer immunity. Cell Death Differ, 15, $80-88$.

Inoue, K. (2005). PLP1-related inherited dysmyelinating disorders: Pelizaeus-Merzbacher disease and spastic paraplegia type 2. Neurogenetics, 6, 1-16.

Ito, H., Murakami, M., Furuhata, A., Gao, S., Yoshida, K., Sobue, S., Hagiwara, K., Takagi, A., Kojima, T., Suzuki, M., Banno, Y., Tanaka, K., Tamiya-Koizumi, K., Kyogashima, M., Nozawa, Y., \& Murate, T. (2009). Transcriptional regulation of neutral sphingomyelinase 2 gene expression of a human breast cancer cell line, MCF-7, induced by the anti-cancer drug, daunorubicin. Biochim Biophys Acta, 1789, 681-690.

Itoh, T. \& Fukuda, M. (2006). Identification of EPI64 as a GTPase-activating protein specific for Rab27A. J Biol Chem, 281, 31823-31831.

Itoh, T., Satoh, M., Kanno, E., \& Fukuda, M. (2006). Screening for target Rabs of TBC (Tre2/Bub2/Cdc16) domain-containing proteins based on their Rab-binding activity.has. Genes Cells, 11, 1023-1037.

Jahn, R. \& Scheller, R. H. (2006). SNAREs-engines for membrane fusion. Nat Rev Mol Cell Biol, $7,631-643$.

Johnstone, R. M., Adam, M., Hammond, J. R., Orr, L., \& Turbide, C. (1987). Vesicle formation during reticulocyte maturation. Association of plasma membrane activities with released vesicles (exosomes). J Biol Chem, 262, 9412-9420.

Johnstone, R. M., Bianchini, A., \& Teng, K. (1989). Reticulocyte maturation and exosome release: transferrin receptor containing exosomes shows multiple plasma membrane functions. Blood, $74,1844-1851$.

Jung, M., Krämer, E., Grzenkowski, M., Tang, K., Blakemore, W., Aguzzi, A., Khazaie, K., Chlichlia, K., von Blankenfeld, G., \& Kettenmann, H. (1995). Lines of murine oligodendroglial precursor cells immortalized by an activated neu tyrosine kinase show distinct degrees of interaction with axons in vitro and in vivo. Eur J Neurosci, 7, 1245-1265. 
Karacostas, V., Nagashima, K., Gonda, M. A., \& Moss, B. (1989). Human immunodeficiency virus-like particles produced by a vaccinia virus expression vector. Proc Natl Acad Sci U S A, 86, 8964-8967.

Katzmann, D. J., Babst, M., \& Emr, S. D. (2001). Ubiquitin-dependent sorting into the multivesicular body pathway requires the function of a conserved endosomal protein sorting complex, ESCRT-I. Cell, 106, 145-155.

Katzmann, D. J., Odorizzi, G., \& Emr, S. D. (2002). Receptor downregulation and multivesicularbody sorting. Nat Rev Mol Cell Biol, 3, 893-905.

Keller, S., König, A.-K., Marmé, F., Runz, S., Wolterink, S., Koensgen, D., Mustea, A., Sehouli, J., \& Altevogt, P. (2009). Systemic presence and tumor-growth promoting effect of ovarian carcinoma released exosomes. Cancer Lett, 278, 73-81.

Kiessling, V., Wan, C., \& Tamm, L. K. (2009). Domain coupling in asymmetric lipid bilayers. Biochim Biophys Acta, 1788, 64-71.

Klugmann, M., Schwab, M. H., Pühlhofer, A., Schneider, A., Zimmermann, F., Griffiths, I. R., \& Nave, K. A. (1997). Assembly of CNS myelin in the absence of proteolipid protein. Neuron, 18, $59-70$.

Komada, M. \& Soriano, P. (1999). Hrs, a FYVE finger protein localized to early endosomes, is implicated in vesicular traffic and required for ventral folding morphogenesis. Genes Dev, 13, $1475-1485$.

Kooijman, E. E., Chupin, V., Fuller, N. L., Kozlov, M. M., de Kruijff, B., Burger, K. N. J., \& Rand, P. R. (2005). Spontaneous curvature of phosphatidic acid and lysophosphatidic acid. Biochemistry, 44, 2097-2102.

Kornfeld, S. \& Mellman, I. (1989). The biogenesis of lysosomes. Annu Rev Cell Biol, 5, 483-525.

Kostelansky, M. S., Schluter, C., Tam, Y. Y. C., Lee, S., Ghirlando, R., Beach, B., Conibear, E., \& Hurley, J. H. (2007). Molecular architecture and functional model of the complete yeast ESCRT-I heterotetramer. Cell, 129, 485-498.

Kouranti, I., Sachse, M., Arouche, N., Goud, B., \& Echard, A. (2006). Rab35 regulates an endocytic recycling pathway essential for the terminal steps of cytokinesis. Curr Biol, 16, 1719-1725.

Krut, O., Wiegmann, K., Kashkar, H., Yazdanpanah, B., \& Krönke, M. (2006). Novel tumor necrosis factor-responsive mammalian neutral sphingomyelinase-3 is a C-tail-anchored protein. J Biol Chem, 281, 13784-13793.

Krämer-Albers, E.-M., Bretz, N., Tenzer, S., Winterstein, C., Möbius, W., Berger, H., Nave, K.-A., Schild, H., \& Trotter, J. (2007). Oligodendrocytes secrete exosomes containing major myelin and stress-protective proteins: Trophic support for axons? PROTEOMICS - CLINICAL APPLICATIONS, 1, 1446-1461. 
Kuroda, T. S. \& Fukuda, M. (2004). Rab27A-binding protein Slp2-a is required for peripheral melanosome distribution and elongated cell shape in melanocytes. Nat Cell Biol, 6, 1195-1203.

Kuroda, T. S., Fukuda, M., Ariga, H., \& Mikoshiba, K. (2002). The Slp homology domain of synaptotagmin-like proteins 1-4 and Slac2 functions as a novel Rab27A binding domain. J Biol Chem, 277, 9212-9218.

Lanzetti, L., Rybin, V., Malabarba, M. G., Christoforidis, S., Scita, G., Zerial, M., \& Fiore, P. P. D. (2000). The Eps8 protein coordinates EGF receptor signalling through Rac and trafficking through Rab5. Nature, 408, 374-377.

Larocca, J. N. \& Norton, W. T. (2007). Isolation of myelin. Curr Protoc Cell Biol, Chapter 3, Unit3.25.

Lata, S., Schoehn, G., Jain, A., Pires, R., Piehler, J., Gottlinger, H. G., \& Weissenhorn, W. (2008). Helical structures of ESCRT-III are disassembled by VPS4. Science, 321, 1354-1357.

Laulagnier, K., Motta, C., Hamdi, S., Roy, S., Fauvelle, F., Pageaux, J.-F., Kobayashi, T., Salles, J.-P., Perret, B., Bonnerot, C., \& Record, M. (2004). Mast cell- and dendritic cell-derived exosomes display a specific lipid composition and an unusual membrane organization. Biochem J, 380, 161-171.

Lauwers, E., Jacob, C., \& André, B. (2009). K63-linked ubiquitin chains as a specific signal for protein sorting into the multivesicular body pathway. J Cell Biol, 185, 493-502.

Lee, S., Joshi, A., Nagashima, K., Freed, E. O., \& Hurley, J. H. (2007). Structural basis for viral late-domain binding to Alix. Nat Struct Mol Biol, 14, 194-199.

Leung, D. W. (2001). The structure and functions of human lysophosphatidic acid acyltransferases. Front Biosci, 6, D944-D953.

Leung, K. F., Dacks, J. B., \& Field, M. C. (2008). Evolution of the multivesicular body ESCRT machinery; retention across the eukaryotic lineage. Traffic, 9, 1698-1716.

Li, R., Blanchette-Mackie, E. J., \& Ladisch, S. (1999). Induction of endocytic vesicles by exogenous C(6)-ceramide. J Biol Chem, 274, 21121-21127.

Liu, C., Yu, S., Zinn, K., Wang, J., Zhang, L., Jia, Y., Kappes, J. C., Barnes, S., Kimberly, R. P., Grizzle, W. E., \& Zhang, H.-G. (2006). Murine mammary carcinoma exosomes promote tumor growth by suppression of NK cell function. J Immunol, 176, 1375-1385.

Lloyd, T. E., Atkinson, R., Wu, M. N., Zhou, Y., Pennetta, G., \& Bellen, H. J. (2002). Hrs regulates endosome membrane invagination and tyrosine kinase receptor signaling in Drosophila. Cell, 108, 261-269.

Malerød, L., Stuffers, S., Brech, A., \& Stenmark, H. (2007). Vps22/EAP30 in ESCRT-II mediates endosomal sorting of growth factor and chemokine receptors destined for lysosomal degradation. Traffic, 8, 1617-1629. 
Marchant, R., Peat, A., \& Banbury, G. H. (1967). The Ultrastructural Basis of Hyphal Growth. New Phytologist, 66, 623-629.

Martin-Serrano, J., Zang, T., \& Bieniasz, P. D. (2003). Role of ESCRT-I in retroviral budding. J Virol, 77, 4794-4804.

Martinu, L., Santiago-Walker, A., Qi, H., \& Chou, M. M. (2002). Endocytosis of epidermal growth factor receptor regulated by Grb2-mediated recruitment of the Rab5 GTPase-activating protein RN-tre. J Biol Chem, 277, 50996-51002.

Masson, D. \& Tschopp, J. (1985). Isolation of a lytic, pore-forming protein (perforin) from cytolytic T-lymphocytes. J Biol Chem, 260, 9069-9072.

Matsui, Y., Kikuchi, A., Araki, S., Hata, Y., Kondo, J., Teranishi, Y., \& Takai, Y. (1990). Molecular cloning and characterization of a novel type of regulatory protein (GDI) for smg p25A, a ras p21-like GTP-binding protein. Mol Cell Biol, 10, 4116-4122.

Matsuo, H., Chevallier, J., Mayran, N., Blanc, I. L., Ferguson, C., Fauré, J., Blanc, N. S., Matile, S., Dubochet, J., Sadoul, R., Parton, R. G., Vilbois, F., \& Gruenberg, J. (2004). Role of LBPA and Alix in multivesicular liposome formation and endosome organization. Science, 303, $531-534$.

Maxfield, F. R. \& McGraw, T. E. (2004). Endocytic recycling. Nat Rev Mol Cell Biol, 5, 121-132.

McBride, H. M., Rybin, V., Murphy, C., Giner, A., Teasdale, R., \& Zerial, M. (1999). Oligomeric complexes link Rab5 effectors with NSF and drive membrane fusion via interactions between EEA1 and syntaxin 13. Cell, 98, 377-386.

McCullough, J., Row, P. E., Lorenzo, O., Doherty, M., Beynon, R., Clague, M. J., \& Urbé, S. (2006). Activation of the endosome-associated ubiquitin isopeptidase AMSH by STAM, a component of the multivesicular body-sorting machinery. Curr Biol, 16, 160-165.

McMahon, H. T. \& Gallop, J. L. (2005). Membrane curvature and mechanisms of dynamic cell membrane remodelling. Nature, 438, 590-596.

Michael, A., Bajracharya, S. D., Yuen, P. S. T., Zhou, H., Star, R. A., Illei, G. G., \& Alevizos, I. (2009). Exosomes from human saliva as a source of microRNA biomarkers. Oral Dis.

Mignot, G., Roux, S., Thery, C., Ségura, E., \& Zitvogel, L. (2006). Prospects for exosomes in immunotherapy of cancer. J Cell Mol Med, 10, 376-388.

Mitchell, P., Petfalski, E., Shevchenko, A., Mann, M., \& Tollervey, D. (1997). The exosome: a conserved eukaryotic RNA processing complex containing multiple 3'-i5' exoribonucleases. Cell, $91,457-466$.

Miyanishi, M., Tada, K., Koike, M., Uchiyama, Y., Kitamura, T., \& Nagata, S. (2007). Identification of Tim4 as a phosphatidylserine receptor. Nature, 450, 435-439. 
Mullock, B. M., Bright, N. A., Fearon, C. W., Gray, S. R., \& Luzio, J. P. (1998). Fusion of lysosomes with late endosomes produces a hybrid organelle of intermediate density and is NSF dependent. J Cell Biol, 140, 591-601.

Muntasell, A., Berger, A. C., \& Roche, P. A. (2007). T cell-induced secretion of MHC class II-peptide complexes on B cell exosomes. EMBO J, 26, 4263-4272.

Muriaux, D., Mirro, J., Harvin, D., \& Rein, A. (2001). RNA is a structural element in retrovirus particles. Proc Natl Acad Sci U S A, 98, 5246-5251.

Möbius, W., Ohno-Iwashita, Y., van Donselaar, E. G., Oorschot, V. M. J., Shimada, Y., Fujimoto, T., Heijnen, H. F. G., Geuze, H. J., \& Slot, J. W. (2002). Immunoelectron microscopic localization of cholesterol using biotinylated and non-cytolytic perfringolysin O. J Histochem Cytochem, 50, 43-55.

Nave, K. A., Lai, C., Bloom, F. E., \& Milner, R. J. (1987). Splice site selection in the proteolipid protein (PLP) gene transcript and primary structure of the DM-20 protein of central nervous system myelin. Proc Natl Acad Sci U S A, 84, 5665-5669.

Neeft, M., Wieffer, M., de Jong, A. S., Negroiu, G., Metz, C. H. G., van Loon, A., Griffith, J., Krijgsveld, J., Wulffraat, N., Koch, H., Heck, A. J. R., Brose, N., Kleijmeer, M., \& van der Sluijs, P. (2005). Munc13-4 is an effector of rab27a and controls secretion of lysosomes in hematopoietic cells. Mol Biol Cell, 16, 731-741.

Nguyen, D. G., Booth, A., Gould, S. J., \& Hildreth, J. E. K. (2003). Evidence that HIV budding in primary macrophages occurs through the exosome release pathway. J Biol Chem, 278, 5234752354 .

Nilsson, B. O., Lennartsson, L., Carlsson, L., Nilsson, S., \& Ronquist, G. (1999). Expression of prostasome-like granules by the prostate cancer cell lines PC3, Du145 and LnCaP grown in monolayer. Ups J Med Sci, 104, 199-206.

Notka, F., Stahl-Hennig, C., Dittmer, U., Wolf, H., \& Wagner, R. (1999). Construction and characterization of recombinant VLPs and Semliki-Forest virus live vectors for comparative evaluation in the SHIV monkey model. Biol Chem, 380, 341-352.

Ohkuma, S. \& Poole, B. (1978). Fluorescence probe measurement of the intralysosomal pH in living cells and the perturbation of pH by various agents. Proc Natl Acad Sci U S A, 75, 3327-3331.

Ostrowski, M., Carmo, N. B., Krumeich, S., Fanget, I., Raposo, G., Savina, A., Moita, C. F., Schauer, K., Hume, A. N., Freitas, R. P., Goud, B., Benaroch, P., Hacohen, N., Fukuda, M., Desnos, C., Seabra, M. C., Darchen, F., Amigorena, S., Moita, L. F., \& Thery, C. (2009). Rab27a and Rab27b control different steps of the exosome secretion pathway. Nat Cell Biol.

Pan, B. T. \& Johnstone, R. (1984). Selective externalization of the transferrin receptor by sheep reticulocytes in vitro. Response to ligands and inhibitors of endocytosis. J Biol Chem, 259, 9776-9782. 
Pan, B. T. \& Johnstone, R. M. (1983). Fate of the transferrin receptor during maturation of sheep reticulocytes in vitro: selective externalization of the receptor. Cell, 33, 967-978.

Pan, X., Eathiraj, S., Munson, M., \& Lambright, D. G. (2006). TBC-domain GAPs for Rab GTPases accelerate GTP hydrolysis by a dual-finger mechanism. Nature, 442, 303-306.

Pasternack, M. S., Bleier, K. J., \& McInerney, T. N. (1991). Granzyme A binding to target cell proteins. Granzyme A binds to and cleaves nucleolin in vitro. J Biol Chem, 266, 14703-14708.

Patino-Lopez, G., Dong, X., Ben-Aissa, K., Bernot, K. M., Itoh, T., Fukuda, M., Kruhlak, M. J., Samelson, L. E., \& Shaw, S. (2008). Rab35 and its GAP EPI64C in T cells regulate receptor recycling and immunological synapse formation. J Biol Chem, 283, 18323-18330.

Pavoine, C. \& Pecker, F. (2009). Sphingomyelinases: their regulation and roles in cardiovascular pathophysiology. Cardiovasc Res, 82, 175-183.

Pelham, H. R. (2001). SNAREs and the specificity of membrane fusion. Trends Cell Biol, 11, 99-101.

Pereira-Leal, J. B. \& Seabra, M. C. (2000). The mammalian Rab family of small GTPases: definition of family and subfamily sequence motifs suggests a mechanism for functional specificity in the Ras superfamily. J Mol Biol, 301, 1077-1087.

Pfeffer, S. \& Aivazian, D. (2004). Targeting Rab GTPases to distinct membrane compartments. Nat Rev Mol Cell Biol, 5, 886-896.

Pincetic, A. \& Leis, J. (2009). The Mechanism of Budding of Retroviruses From Cell Membranes. Adv Virol, 2009, 6239691-6239699.

Pincetic, A., Medina, G., Carter, C., \& Leis, J. (2008). Avian sarcoma virus and human immunodeficiency virus, type 1 use different subsets of ESCRT proteins to facilitate the budding process. J Biol Chem, 283, 29822-29830.

Piper, R. C. \& Luzio, J. P. (2001). Late endosomes: sorting and partitioning in multivesicular bodies. Traffic, 2, 612-621.

Pisitkun, T., Shen, R.-F., \& Knepper, M. A. (2004). Identification and proteomic profiling of exosomes in human urine. Proc Natl Acad Sci U S A, 101, 13368-13373.

Podack, E. R., Young, J. D., \& Cohn, Z. A. (1985). Isolation and biochemical and functional characterization of perforin 1 from cytolytic T-cell granules. Proc Natl Acad Sci U S A, 82, 8629-8633.

Pons, V., Luyet, P.-P., Morel, E., Abrami, L., van der Goot, F. G., Parton, R. G., \& Gruenberg, J. (2008). Hrs and SNX3 functions in sorting and membrane invagination within multivesicular bodies. PLoS Biol, 6, e214.

Popov, S., Popova, E., Inoue, M., \& Göttlinger, H. G. (2008). Human immunodeficiency virus type 1 Gag engages the Bro1 domain of ALIX/AIP1 through the nucleocapsid. J Virol, 82, 1389-1398. 
Pornillos, O., Alam, S. L., Davis, D. R., \& Sundquist, W. I. (2002). Structure of the Tsg101 UEV domain in complex with the PTAP motif of the HIV-1 p6 protein. Nat Struct Biol, 9, 812-817.

Pornillos, O., Higginson, D. S., Stray, K. M., Fisher, R. D., Garrus, J. E., Payne, M., He, G.-P., Wang, H. E., Morham, S. G., \& Sundquist, W. I. (2003). HIV Gag mimics the Tsg101-recruiting activity of the human Hrs protein. J Cell Biol, 162, 425-434.

Potolicchio, I., Carven, G. J., Xu, X., Stipp, C., Riese, R. J., Stern, L. J., \& Santambrogio, L. (2005). Proteomic analysis of microglia-derived exosomes: metabolic role of the aminopeptidase CD13 in neuropeptide catabolism. J Immunol, 175, 2237-2243.

Prusiner, S. B. (1982). Novel proteinaceous infectious particles cause scrapie. Science, 216, 136-144.

Rabinowits, G., Gerçel-Taylor, C., Day, J. M., Taylor, D. D., \& Kloecker, G. H. (2009). Exosomal microRNA: a diagnostic marker for lung cancer. Clin Lung Cancer, 10, 42-46.

Raiborg, C., Bache, K. G., Gillooly, D. J., Madshus, I. H., Stang, E., \& Stenmark, H. (2002). Hrs sorts ubiquitinated proteins into clathrin-coated microdomains of early endosomes. Nat Cell Biol, 4, 394-398.

Raiborg, C., Bache, K. G., Mehlum, A., Stang, E., \& Stenmark, H. (2001). Hrs recruits clathrin to early endosomes. EMBO J, 20, 5008-5021.

Raiborg, C. \& Stenmark, H. (2009). The ESCRT machinery in endosomal sorting of ubiquitylated membrane proteins. Nature, 458, 445-452.

Raiborg, C., Wesche, J., Malerød, L., \& Stenmark, H. (2006). Flat clathrin coats on endosomes mediate degradative protein sorting by scaffolding Hrs in dynamic microdomains. J Cell Sci, $119,2414-2424$.

Raposo, G. \& Marks, M. S. (2002). The dark side of lysosome-related organelles: specialization of the endocytic pathway for melanosome biogenesis. Traffic, 3, 237-248.

Raposo, G., Marks, M. S., \& Cutler, D. F. (2007). Lysosome-related organelles: driving post-Golgi compartments into specialisation. Curr Opin Cell Biol, 19, 394-401.

Raposo, G., Nijman, H. W., Stoorvogel, W., Liejendekker, R., Harding, C. V., Melief, C. J., \& Geuze, H. J. (1996). B lymphocytes secrete antigen-presenting vesicles. J Exp Med, 183, $1161-1172$.

Raposo, G., Tenza, D., Mecheri, S., Peronet, R., Bonnerot, C., \& Desaymard, C. (1997). Accumulation of major histocompatibility complex class II molecules in mast cell secretory granules and their release upon degranulation. Mol Biol Cell, 8, 2631-2645.

Razi, M. \& Futter, C. E. (2006). Distinct roles for Tsg101 and Hrs in multivesicular body formation and inward vesiculation. Mol Biol Cell, 17, 3469-3483.

Rink, J., Ghigo, E., Kalaidzidis, Y., \& Zerial, M. (2005). Rab conversion as a mechanism of progression from early to late endosomes. Cell, 122, 735-749. 
Rios, J. C., Melendez-Vasquez, C. V., Einheber, S., Lustig, M., Grumet, M., Hemperly, J., Peles, E., \& Salzer, J. L. (2000). Contactin-associated protein (Caspr) and contactin form a complex that is targeted to the paranodal junctions during myelination. J Neurosci, 20, 8354-8364.

Robertson, C., Booth, S. A., Beniac, D. R., Coulthart, M. B., Booth, T. F., \& McNicol, A. (2006). Cellular prion protein is released on exosomes from activated platelets. Blood, 107, 3907-3911.

Rulli, S. J., Hibbert, C. S., Mirro, J., Pederson, T., Biswal, S., \& Rein, A. (2007). Selective and nonselective packaging of cellular RNAs in retrovirus particles. J Virol, 81, 6623-6631.

Safaei, R., Larson, B. J., Cheng, T. C., Gibson, M. A., Otani, S., Naerdemann, W., \& Howell, S. B. (2005). Abnormal lysosomal trafficking and enhanced exosomal export of cisplatin in drug-resistant human ovarian carcinoma cells. Mol Cancer Ther, 4, 1595-1604.

Saftig, P. \& Klumperman, J. (2009). Lysosome biogenesis and lysosomal membrane proteins: trafficking meets function. Nat Rev Mol Cell Biol, 10, 623-635.

Saksena, S., Wahlman, J., Teis, D., Johnson, A. E., \& Emr, S. D. (2009). Functional reconstitution of ESCRT-III assembly and disassembly. Cell, 136, 97-109.

Salzer, J. L. (2003). Polarized domains of myelinated axons. Neuron, 40, 297-318.

Salzer, U. \& Prohaska, R. (2001). Stomatin, flotillin-1, and flotillin-2 are major integral proteins of erythrocyte lipid rafts. Blood, 97, 1141-1143.

Sato, M., Sato, K., Liou, W., Pant, S., Harada, A., \& Grant, B. D. (2008). Regulation of endocytic recycling by C. elegans Rab35 and its regulator RME-4, a coated-pit protein. EMBO J, 27, $1183-1196$.

Savina, A., Fader, C. M., Damiani, M. T., \& Colombo, M. I. (2005). Rab11 promotes docking and fusion of multivesicular bodies in a calcium-dependent manner. Traffic, 6, 131-143.

Savina, A., Furlán, M., Vidal, M., \& Colombo, M. I. (2003). Exosome release is regulated by a calcium-dependent mechanism in K562 cells. J Biol Chem, 278, 20083-20090.

Savina, A., Vidal, M., \& Colombo, M. I. (2002). The exosome pathway in K562 cells is regulated by Rab11. J Cell Sci, 115, 2505-2515.

Schmid, J. A., Ellinger, I., \& Kosma, P. (1998). In vitro fusion of tissue-derived endosomes and lysosomes. Eur J Cell Biol, 77, 166-174.

Schmidt, J. A. \& Brown, W. J. (2009). Lysophosphatidic acid acyltransferase 3 regulates Golgi complex structure and function. J Cell Biol, 186, 211-218.

Schuchman, E. H., Suchi, M., Takahashi, T., Sandhoff, K., \& Desnick, R. J. (1991). Human acid sphingomyelinase. Isolation, nucleotide sequence and expression of the full-length and alternatively spliced cDNAs. J Biol Chem, 266, 8531-8539.

Seabra, M. C. \& Coudrier, E. (2004). Rab GTPases and myosin motors in organelle motility. Traffic, 5, 393-399. 
Segura, E., Guérin, C., Hogg, N., Amigorena, S., \& Théry, C. (2007). CD8+ dendritic cells use LFA-1 to capture MHC-peptide complexes from exosomes in vivo. J Immunol, 179, 1489-1496.

Segura, E., Nicco, C., Lombard, B., Véron, P., Raposo, G., Batteux, F., Amigorena, S., \& Théry, C. (2005). ICAM-1 on exosomes from mature dendritic cells is critical for efficient naive T-cell priming. Blood, 106, 216-223.

Seiberg, M. (2001). Keratinocyte-melanocyte interactions during melanosome transfer. Pigment Cell Res, 14, 236-242.

Sherman, D. L. \& Brophy, P. J. (2005). Mechanisms of axon ensheathment and myelin growth. Nat Rev Neurosci, 6, 683-690.

Shi, L., Kraut, R. P., Aebersold, R., \& Greenberg, A. H. (1992). A natural killer cell granule protein that induces DNA fragmentation and apoptosis. J Exp Med, 175, 553-566.

Shirakawa, R., Higashi, T., Tabuchi, A., Yoshioka, A., Nishioka, H., Fukuda, M., Kita, T., \& Horiuchi, H. (2004). Munc13-4 is a GTP-Rab27-binding protein regulating dense core granule secretion in platelets. J Biol Chem, 279, 10730-10737.

Shirane, M. \& Nakayama, K. I. (2006). Protrudin induces neurite formation by directional membrane trafficking. Science, 314, 818-821.

Simons, M., Krämer, E. M., Thiele, C., Stoffel, W., \& Trotter, J. (2000). Assembly of myelin by association of proteolipid protein with cholesterol- and galactosylceramide-rich membrane domains. J Cell Biol, 151, 143-154.

Simons, M. \& Trotter, J. (2007). Wrapping it up: the cell biology of myelination. Curr Opin Neurobiol, 17, 533-540.

Simpson, R. J., Jensen, S. S., \& Lim, J. W. E. (2008). Proteomic profiling of exosomes: current perspectives. Proteomics, 8, 4083-4099.

Slagsvold, T., Aasland, R., Hirano, S., Bache, K. G., Raiborg, C., Trambaiolo, D., Wakatsuki, S., \& Stenmark, H. (2005). Eap45 in mammalian ESCRT-II binds ubiquitin via a phosphoinositideinteracting GLUE domain. J Biol Chem, 280, 19600-19606.

Smit, N. P., van Roermund, C. W., Aerts, H. M., Heikoop, J. C., den Berg, M. V., Pavel, S., \& Wanders, R. J. (1993). Subcellular fractionation of cultured normal human melanocytes: new insights into the relationship of melanosomes with lysosomes and peroxisomes. Biochim Biophys Acta, 1181, 1-6.

Soldati, T., Shapiro, A. D., Svejstrup, A. B., \& Pfeffer, S. R. (1994). Membrane targeting of the small GTPase Rab9 is accompanied by nucleotide exchange. Nature, 369, 76-78.

Sorkin, A. \& Goh, L. K. (2009). Endocytosis and intracellular trafficking of ErbBs. Exp Cell Res, 315, 683-696. 
Spitzer, C., Schellmann, S., Sabovljevic, A., Shahriari, M., Keshavaiah, C., Bechtold, N., Herzog, M., Müller, S., Hanisch, F.-G., \& Hülskamp, M. (2006). The Arabidopsis elch mutant reveals functions of an ESCRT component in cytokinesis. Development, 133, 4679-4689.

Steinman, R. M. (1991). The dendritic cell system and its role in immunogenicity. Annu Rev Immunol, 9, 271-296.

Stenmark, H. (2009). Rab GTPases as coordinators of vesicle traffic. Nat Rev Mol Cell Biol, 10, $513-525$.

Stenmark, H., Parton, R. G., Steele-Mortimer, O., Lütcke, A., Gruenberg, J., \& Zerial, M. (1994). Inhibition of rab5 GTPase activity stimulates membrane fusion in endocytosis. EMBO J, 13, $1287-1296$.

Stinchcombe, J. C., Bossi, G., Booth, S., \& Griffiths, G. M. (2001). The immunological synapse of CTL contains a secretory domain and membrane bridges. Immunity, 15, 751-761.

Stoffel, W., Jenke, B., Blöck, B., Zumbansen, M., \& Koebke, J. (2005). Neutral sphingomyelinase 2 (smpd3) in the control of postnatal growth and development. Proc Natl Acad Sci U S A, 102, $4554-4559$.

Stoffel, W., Jenke, B., Holz, B., Binczek, E., Günter, R. H., Knifka, J., Koebke, J., \& Niehoff, A. (2007). Neutral sphingomyelinase (SMPD3) deficiency causes a novel form of chondrodysplasia and dwarfism that is rescued by Col2A1-driven smpd3 transgene expression. Am J Pathol, 171, $153-161$.

Stoorvogel, W., Oorschot, V., \& Geuze, H. J. (1996). A novel class of clathrin-coated vesicles budding from endosomes. J Cell Biol, 132, 21-33.

Stoorvogel, W., Strous, G. J., Geuze, H. J., Oorschot, V., \& Schwartz, A. L. (1991). Late endosomes derive from early endosomes by maturation. Cell, 65, 417-427.

Strack, B., Calistri, A., Craig, S., Popova, E., \& Göttlinger, H. G. (2003). AIP1/ALIX is a binding partner for HIV-1 p6 and EIAV p9 functioning in virus budding. Cell, 114, 689-699.

Strom, M., Hume, A. N., Tarafder, A. K., Barkagianni, E., \& Seabra, M. C. (2002). A family of Rab27-binding proteins. Melanophilin links Rab27a and myosin Va function in melanosome transport. J Biol Chem, 277, 25423-25430.

Stuffers, S., Wegner, C. S., Stenmark, H., \& Brech, A. (2009). Multivesicular endosome biogenesis in the absence of ESCRTs. Traffic, 10, 925-937.

Subra, C., Laulagnier, K., Perret, B., \& Record, M. (2007). Exosome lipidomics unravels lipid sorting at the level of multivesicular bodies. Biochimie, 89, 205-212.

Sweitzer, S. M. \& Hinshaw, J. E. (1998). Dynamin undergoes a GTP-dependent conformational change causing vesiculation. Cell, 93, 1021-1029.

Sztul, E. \& Lupashin, V. (2006). Role of tethering factors in secretory membrane traffic. Am J Physiol Cell Physiol, 290, C11-C26. 
Sönnichsen, B., Renzis, S. D., Nielsen, E., Rietdorf, J., \& Zerial, M. (2000). Distinct membrane domains on endosomes in the recycling pathway visualized by multicolor imaging of Rab4, Rab5, and Rab11. J Cell Biol, 149, 901-914.

Taieb, J., Chaput, N., Schartz, N., Roux, S., Novault, S., Ménard, C., Ghiringhelli, F., Terme, M., Carpentier, A. F., Darrasse-Jèze, G., Darrasse-Jèse, G., Lemonnier, F., \& Zitvogel, L. (2006). Chemoimmunotherapy of tumors: cyclophosphamide synergizes with exosome based vaccines. J Immunol, 176, 2722-2729.

Tanaka, N., Kyuuma, M., \& Sugamura, K. (2008). Endosomal sorting complex required for transport proteins in cancer pathogenesis, vesicular transport, and non-endosomal functions. Cancer Sci, 99, 1293-1303.

Tani, M. \& Hannun, Y. A. (2007a). Analysis of membrane topology of neutral sphingomyelinase 2. FEBS Lett, 581, 1323-1328.

Tani, M. \& Hannun, Y. A. (2007b). Neutral sphingomyelinase 2 is palmitoylated on multiple cysteine residues. Role of palmitoylation in subcellular localization. J Biol Chem, 282, 1004710056 .

Taylor, D. D. \& Gercel-Taylor, C. (2008). MicroRNA signatures of tumor-derived exosomes as diagnostic biomarkers of ovarian cancer. Gynecol Oncol, 110, 13-21.

Teis, D., Saksena, S., \& Emr, S. D. (2008). Ordered assembly of the ESCRT-III complex on endosomes is required to sequester cargo during MVB formation. Dev Cell, 15, 578-589.

Teo, H., Gill, D. J., Sun, J., Perisic, O., Veprintsev, D. B., Vallis, Y., Emr, S. D., \& Williams, R. L. (2006). ESCRT-I core and ESCRT-II GLUE domain structures reveal role for GLUE in linking to ESCRT-I and membranes. Cell, 125, 99-111.

Theos, A. C., Truschel, S. T., Tenza, D., Hurbain, I., Harper, D. C., Berson, J. F., Thomas, P. C., Raposo, G., \& Marks, M. S. (2006). A lumenal domain-dependent pathway for sorting to intralumenal vesicles of multivesicular endosomes involved in organelle morphogenesis. Dev Cell, 10, 343-354.

Théry, C., Boussac, M., Véron, P., Ricciardi-Castagnoli, P., Raposo, G., Garin, J., \& Amigorena, S. (2001). Proteomic analysis of dendritic cell-derived exosomes: a secreted subcellular compartment distinct from apoptotic vesicles. J Immunol, 166, 7309-7318.

Théry, C., Duban, L., Segura, E., Véron, P., Lantz, O., \& Amigorena, S. (2002a). Indirect activation of naïve CD4+ T cells by dendritic cell-derived exosomes. Nat Immunol, 3, 1156-1162.

Théry, C., Zitvogel, L., \& Amigorena, S. (2002b). Exosomes: composition, biogenesis and function. Nat Rev Immunol, 2, 569-579.

Tooze, J. \& Hollinshead, M. (1991). Tubular early endosomal networks in AtT20 and other cells. J Cell Biol, 115, 635-653. 
Trajkovic, K., Dhaunchak, A. S., Goncalves, J. T., Wenzel, D., Schneider, A., Bunt, G., Nave, K.-A., \& Simons, M. (2006). Neuron to glia signaling triggers myelin membrane exocytosis from endosomal storage sites. J Cell Biol, 172, 937-948.

Trams, E. G., Lauter, C. J., Salem, N., \& Heine, U. (1981). Exfoliation of membrane ecto-enzymes in the form of micro-vesicles. Biochim Biophys Acta, 645, 63-70.

Trapp, B. D., Andrews, S. B., Cootauco, C., \& Quarles, R. (1989). The myelin-associated glycoprotein is enriched in multivesicular bodies and periaxonal membranes of actively myelinating oligodendrocytes. J Cell Biol, 109, 2417-2426.

Tritel, M. \& Resh, M. D. (2001). The late stage of human immunodeficiency virus type 1 assembly is an energy-dependent process. J Virol, 75, 5473-5481.

Tytell, M. (2005). Release of heat shock proteins (Hsps) and the effects of extracellular Hsps on neural cells and tissues. Int J Hyperthermia, 21, 445-455.

Ullrich, O., Horiuchi, H., Bucci, C., \& Zerial, M. (1994). Membrane association of Rab5 mediated by GDP-dissociation inhibitor and accompanied by GDP/GTP exchange. Nature, 368, 157-160.

Ullrich, O., Stenmark, H., Alexandrov, K., Huber, L. A., Kaibuchi, K., Sasaki, T., Takai, Y., \& Zerial, M. (1993). Rab GDP dissociation inhibitor as a general regulator for the membrane association of rab proteins. J Biol Chem, 268, 18143-18150.

Usami, Y., Popov, S., Popova, E., \& Göttlinger, H. G. (2008). Efficient and specific rescue of human immunodeficiency virus type 1 budding defects by a Nedd4-like ubiquitin ligase. J Virol, $82,4898-4907$.

Usami, Y., Popov, S., Popova, E., Inoue, M., Weissenhorn, W., \& Göttlinger, H. G. (2009). The ESCRT pathway and HIV-1 budding. Biochem Soc Trans, 37, 181-184.

Utermöhlen, O., Herz, J., Schramm, M., \& Krönke, M. (2008). Fusogenicity of membranes: the impact of acid sphingomyelinase on innate immune responses. Immunobiology, 213, 307-314.

Valadi, H., Ekström, K., Bossios, A., Sjöstrand, M., Lee, J. J., \& Lötvall, J. O. (2007). Exosomemediated transfer of mRNAs and microRNAs is a novel mechanism of genetic exchange between cells. Nat Cell Biol, 9, 654-659.

van Meel, E. \& Klumperman, J. (2008). Imaging and imagination: understanding the endolysosomal system. Histochem Cell Biol, 129, 253-266.

van Niel, G., Porto-Carreiro, I., Simoes, S., \& Raposo, G. (2006). Exosomes: a common pathway for a specialized function. J Biochem, 140, 13-21.

van Niel, G., Raposo, G., Candalh, C., Boussac, M., Hershberg, R., Cerf-Bensussan, N., \& Heyman, M. (2001). Intestinal epithelial cells secrete exosome-like vesicles. Gastroenterology, 121, 337349 . 
Vella, L. J., Greenwood, D. L. V., Cappai, R., Scheerlinck, J.-P. Y., \& Hill, A. F. (2008). Enrichment of prion protein in exosomes derived from ovine cerebral spinal fluid. Vet Immunol Immunopathol, 124, 385-393.

Vella, L. J., Sharples, R. A., Lawson, V. A., Masters, C. L., Cappai, R., \& Hill, A. F. (2007). Packaging of prions into exosomes is associated with a novel pathway of $\operatorname{PrP}$ processing. J Pathol, 211, 582-590.

VerPlank, L., Bouamr, F., LaGrassa, T. J., Agresta, B., Kikonyogo, A., Leis, J., \& Carter, C. A. (2001). Tsg101, a homologue of ubiquitin-conjugating (E2) enzymes, binds the L domain in HIV type 1 Pr55(Gag). Proc Natl Acad Sci U S A, 98, 7724-7729.

Vidal, M., Mangeat, P., \& Hoekstra, D. (1997). Aggregation reroutes molecules from a recycling to a vesicle-mediated secretion pathway during reticulocyte maturation. J Cell Sci, 110 ( Pt 16), $1867-1877$.

Vidal, M., Sainte-Marie, J., Philippot, J. R., \& Bienvenue, A. (1989). Asymmetric distribution of phospholipids in the membrane of vesicles released during in vitro maturation of guinea pig reticulocytes: evidence precluding a role for "aminophospholipid translocase". J Cell Physiol, $140,455-462$.

von Zastrow, M. \& Sorkin, A. (2007). Signaling on the endocytic pathway. Curr Opin Cell Biol, $19,436-445$.

Waguri, S., Dewitte, F., Borgne, R. L., Rouillé, Y., Uchiyama, Y., Dubremetz, J.-F., \& Hoflack, B. (2003). Visualization of TGN to endosome trafficking through fluorescently labeled MPR and AP-1 in living cells. Mol Biol Cell, 14, 142-155.

Walseng, E., Bakke, O., \& Roche, P. A. (2008). Major histocompatibility complex class II-peptide complexes internalize using a clathrin- and dynamin-independent endocytosis pathway. J Biol Chem, 283, 14717-14727.

Wei, S., Gamero, A. M., Liu, J. H., Daulton, A. A., Valkov, N. I., Trapani, J. A., Larner, A. C., Weber, M. J., \& Djeu, J. Y. (1998). Control of lytic function by mitogen-activated protein kinase/extracellular regulatory kinase 2 (ERK2) in a human natural killer cell line: identification of perforin and granzyme B mobilization by functional ERK2. J Exp Med, 187, 1753-1765.

Weiss, B., Jacquemin-Sablon, A., Live, T. R., Fareed, G. C., \& Richardson, C. C. (1968). Enzymatic breakage and joining of deoxyribonucleic acid. VI. Further purification and properties of polynucleotide ligase from Escherichia coli infected with bacteriophage T4. J Biol Chem, 243, $4543-4555$.

Werner, H. B., Kuhlmann, K., Shen, S., Uecker, M., Schardt, A., Dimova, K., Orfaniotou, F., Dhaunchak, A., Brinkmann, B. G., Möbius, W., Guarente, L., Casaccia-Bonnefil, P., Jahn, O., \& Nave, K.-A. (2007). Proteolipid protein is required for transport of sirtuin 2 into CNS myelin. J Neurosci, 27, 7717-7730. 
Westbroek, W., Tuchman, M., Tinloy, B., Wever, O. D., Vilboux, T., Hertz, J. M., Hasle, H., Heilmann, C., Helip-Wooley, A., Kleta, R., \& Gahl, W. A. (2008). A novel missense mutation (G43S) in the switch I region of Rab27A causing Griscelli syndrome. Mol Genet Metab, 94, $248-254$.

Westphal, V., Rizzoli, S. O., Lauterbach, M. A., Kamin, D., Jahn, R., \& Hell, S. W. (2008). Videorate far-field optical nanoscopy dissects synaptic vesicle movement. Science, 320, 246-249.

Wilcke, M., Johannes, L., Galli, T., Mayau, V., Goud, B., \& Salamero, J. (2000). Rab11 regulates the compartmentalization of early endosomes required for efficient transport from early endosomes to the trans-golgi network. J Cell Biol, 151, 1207-1220.

Williams, R. L. \& Urbé, S. (2007). The emerging shape of the ESCRT machinery. Nat Rev Mol Cell Biol, 8, 355-368.

Witting, A., Müller, P., Herrmann, A., Kettenmann, H., \& Nolte, C. (2000). Phagocytic clearance of apoptotic neurons by Microglia/Brain macrophages in vitro: involvement of lectin-, integrin-, and phosphatidylserine-mediated recognition. J Neurochem, 75, 1060-1070.

Wolfers, J., Lozier, A., Raposo, G., Regnault, A., Théry, C., Masurier, C., Flament, C., Pouzieux, S., Faure, F., Tursz, T., Angevin, E., Amigorena, S., \& Zitvogel, L. (2001). Tumor-derived exosomes are a source of shared tumor rejection antigens for CTL cross-priming. Nat Med, 7, $297-303$

Wu, X., Rao, K., Bowers, M. B., Copeland, N. G., Jenkins, N. A., \& Hammer, J. A. (2001). Rab27a enables myosin Va-dependent melanosome capture by recruiting the myosin to the organelle. J Cell Sci, 114, 1091-1100.

Wu, X. S., Rao, K., Zhang, H., Wang, F., Sellers, J. R., Matesic, L. E., Copeland, N. G., Jenkins, N. A., \& Hammer, J. A. (2002). Identification of an organelle receptor for myosin-Va. Nat Cell Biol, 4, 271-278.

Wubbolts, R., Leckie, R. S., Veenhuizen, P. T. M., Schwarzmann, G., Möbius, W., Hoernschemeyer, J., Slot, J.-W., Geuze, H. J., \& Stoorvogel, W. (2003). Proteomic and biochemical analyses of human B cell-derived exosomes. Potential implications for their function and multivesicular body formation. J Biol Chem, 278, 10963-10972.

Yoshimori, T., Yamagata, F., Yamamoto, A., Mizushima, N., Kabeya, Y., Nara, A., Miwako, I., Ohashi, M., Ohsumi, M., \& Ohsumi, Y. (2000). The mouse SKD1, a homologue of yeast Vps4p, is required for normal endosomal trafficking and morphology in mammalian cells. Mol Biol Cell, $11,747-763$.

Yoshimura, S.-I., Egerer, J., Fuchs, E., Haas, A. K., \& Barr, F. A. (2007). Functional dissection of Rab GTPases involved in primary cilium formation. J Cell Biol, 178, 363-369.

Zakharova, L., Svetlova, M., \& Fomina, A. F. (2007). T cell exosomes induce cholesterol accumulation in human monocytes via phosphatidylserine receptor. J Cell Physiol, 212, 174-181. 
Zha, X., Pierini, L. M., Leopold, P. L., Skiba, P. J., Tabas, I., \& Maxfield, F. R. (1998). Sphingomyelinase treatment induces ATP-independent endocytosis. J Cell Biol, 140, 39-47.

Zhai, Q., Fisher, R. D., Chung, H.-Y., Myszka, D. G., Sundquist, W. I., \& Hill, C. P. (2008). Structural and functional studies of ALIX interactions with YPX(n)L late domains of HIV-1 and EIAV. Nat Struct Mol Biol, 15, 43-49.

Zhang, J., Fonovic, M., Suyama, K., Bogyo, M., \& Scott, M. P. (2009). Rab35 controls actin bundling by recruiting fascin as an effector protein. Science, 325, 1250-1254.

Zhang, X.-M., Ellis, S., Sriratana, A., Mitchell, C. A., \& Rowe, T. (2004). Sec15 is an effector for the Rab11 GTPase in mammalian cells. J Biol Chem, 279, 43027-43034.

Zhang, X.-M., Walsh, B., Mitchell, C. A., \& Rowe, T. (2005). TBC domain family, member 15 is a novel mammalian Rab GTPase-activating protein with substrate preference for Rab7. Biochem Biophys Res Commun, 335, 154-161.

Zitvogel, L., Regnault, A., Lozier, A., Wolfers, J., Flament, C., Tenza, D., Ricciardi-Castagnoli, P., Raposo, G., \& Amigorena, S. (1998). Eradication of established murine tumors using a novel cell-free vaccine: dendritic cell-derived exosomes. Nat Med, 4, 594-600.

Zumbansen, M. \& Stoffel, W. (2002). Neutral sphingomyelinase 1 deficiency in the mouse causes no lipid storage disease. Mol Cell Biol, 22, 3633-3638. 


\title{
Curriculum Vitae
}

\author{
Chieh Hsu (徐捷) \\ born on $8^{\text {th }}$ February 1980 in Taipei, Taiwan \\ Hermann-Rein-Str. 3, 37075, Göttingen, Germany \\ Phone +49 (0) 5513899534 \\ E-Mail hsu@em.mpg.de
}

\begin{abstract}
Education
2006 - 2010 Ph.D. Göttingen Graduate School for Neurosciences and Molecular

Biosciences, University of Göttingen, Germany

2004 - 2006 M.Sc. International Max Planck Research School for Molecular Biology

University of Göttingen, Germany

1998 - 2002 B.Sc. Department of Botany

National Taiwan University (國立臺灣大學), Taiwan

1995 - 1998 Taipei Municipal Jianguo High School (臺北市立建國高級中學), Taipei, Taiwan

\section{Scholarships \& Prizes}

2006 - 2009 Stipend Graduate Program 521 of Deutsche Forschungsgemeinschaft "ProteinProtein-Interactions During Intracellular Transport of Macromolecules"

2004 - 2005 Stipend International Max Planck Research School

2002 Dean's Award, National Taiwan University

$1998 \quad$ First Prize of National Contest on Biology (senior high school group)

1995 Second Prize of National Contest on Mathematics (junior high school group)

\section{Publications}

Chieh Hsu, Yuichi Morohashi, Shin-ichiro Yoshimura, Natalia Manrique-Hoyos, SangYong Jung, Marcel A. Lauterbach, Mostafa Bakhti, Mads Grønborg, Wiebke Möbius, JeongSeop Rhee, Francis A. Barr and Mikael Simons. (2010) Regulation of exosome secretion by Rab35 and its GTPaseactivating proteins TBC1D10A-C. J. Cell Biol.; 189:223-232; doi:10.1083/jcb.200911018

Katarina Trajkovic*, Chieh Hsu$^{*}$, Salvatore Chiantia, Lawrence Rajendran, Dirk Wenzel, Felix Wieland, Petra Schwille, Britta Brügger and Mikael Simons. (2008) Ceramide triggers budding of exosome vesicles into multivesicular endosomes. Science; 319:1244-1247; doi:10.1126/science. 1153124
\end{abstract}

*Equal contribution 